\title{
Uniformizing The Moduli Stacks of Global $\mathfrak{G}$-Shtukas
}


Mathematik

\title{
Uniformizing The Moduli Stacks of Global $\mathfrak{G}$-Shtukas
}

\author{
Inaugural-Dissertation \\ zur Erlangung des Doktorgrades \\ der Naturwissenschaften im Fachbereich \\ Mathematik und Informatik \\ der Mathematisch-Naturwissenschaftlichen Fakultät \\ der Westfälischen Wilhelms-Universität Münster
}

vorgelegt von

M. E. Arasteh Rad

aus Teheran

$-2012-$ 
Dekan:

Erster Gutachter:

Zweiter Gutachter:

Tag der mündlichen Prüfung:

Tag der Promotion:
Prof. Dr. Martin Stein

Prof. Dr. Urs Hartl

Prof. Dr. Eva Viehmann

07.12.2012

07.12 .2012 
To My Teachers 


\section{Contents}

1 Introduction $\quad 7$

1.1 Notation and Conventions . . . . . . . . . . . . . 10

2 Glob. and Loc. Shtukas, Def. and Their Moduli Stacks 13

2.1 Formal Algebraic Stacks . . . . . . . . . . . . . . . 13

2.2 Global $\mathfrak{G}$-Shtukas . . . . . . . . . . . . . . . . . . . . . 16

2.3 Loop groups and Local $\mathbb{P}$-Shtukas . . . . . . . . . . . . 18

3 A starting point of the analogy 21

3.1 Rigidity of quasi-isogenies . . . . . . . . . . . . . . . 21

3.2 The Global-Local Functor . . . . . . . . . . . . . . . . . . 23

4 Unbounded Uniformization and The Deformation Theory 27

4.1 Construction of the Uniformization map . . . . . . . . . 27

4.2 The Analogue of the Serre-Tate Theorem . . . . . . . . . . 36

5 Galois Representations and Shtukas 39

5.1 The Tate Functor . . . . . . . . . . . . . . . . . . . . . . . . 39

5.2 The Level Structure . . . . . . . . . . . . . . . . . . . . . 41

6 The Rapoport-Zink Space for Local $\mathbb{P}$-Shtukas 47

6.1 Some discussion about Bruhat-Tits building . . . . . . . . 48

6.2 The boundedness conditions . . . . . . . . . . . . 50

6.2.1 Bounds on the modui of local objects . . . . . . . . 50

6.2.2 Bounds on the moduli of global objects . . . . . . . 56

6.3 Representablity of The Rapoport-Zink Functor . . . . . . . . 61

7 The Uniformization Theorem $\quad 69$

7.1 The Uniformization Theorem . . . . . . . . . . . . . . . 69 
8 Discussion about Uniformization and Local Model $\quad 77$ 8.1 Local model for the moduli of global $\mathfrak{G}$-shtukas . . . . . . . 77

8.2 Generalized Lang Morphism . . . . . . . . . . . . . . . . . 81

$\begin{array}{lc}\text { References } & 83\end{array}$ 


\section{Chapter 1}

\section{Introduction}

Let $\mathfrak{G}$ be a parahoric Bruhat-Tits group scheme over a smooth projective curve $C$ over $\mathbb{F}_{q}$, see [PR3]. A global $\mathfrak{G}$-shtuka $\underline{\mathcal{G}}$ over $S$ in $S c h / \mathbb{F}_{q}$ is a tuple $\left(\mathcal{G}, s_{1}, \ldots, s_{n}, \varphi\right)$ consisting of a $\mathfrak{G}$-bundle $\mathcal{G}$ over $C_{S}:=C \times_{\mathbb{F}_{q}} S$, an $n$-tuple of (characteristic) sections $\left(s_{1}, \ldots, s_{n}\right) \in C^{n}(S)$ and a Frobenius connection $\varphi$ defined outside the graph of the sections $s_{i}$ 's (i.e. an isomorphism $\left.\left.\sigma^{*} \mathcal{G}\right|_{C_{S} \backslash \cup_{i} \Gamma_{s_{i}}} \stackrel{\sim}{\rightarrow} \mathcal{G}\right|_{C_{S} \backslash \cup_{i} \Gamma_{s_{i}}}$ where $\left.\sigma^{*}=\left(i d_{C} \times F r o b_{q}\right)^{*}\right)$.

Spelling out the Riemann-Hilbert correspondence for "function fields", together with the tannakian philosophy, one sees that the stack $\nabla_{n} \mathscr{H}^{1}(C, \mathfrak{G})$ of global $\mathfrak{G}$-shtukas, after imposing suitable boundedness conditions and level structures (as we will explain in chapter 5 and section 6.2), may play the same role that Shimura varieties play in the mixed characteristic set up. More specifically one can hope that the Langlands correspondence for function fields is realized on its cohomology. Note that in particular this moduli stack generalizes the space of $F$-sheaves $F S h_{D, r}$ which was considered by L. Lafforgue (and previously V. Drinfeld) in his proof of the Langlands correspondence for the case that $\mathfrak{G}=G l_{r}$ (resp. $\mathfrak{G}=G l_{2}$ ). On the other hand "this analogy" can be viewed as an attempt to build a bridge between the geometric Langlands program and the arithmetic Langlands program, where the role of global shtukas is played by the abelian varieties (together with additional structures) and $D$-modules respectively.

In this thesis our approach to study the moduli stack of global $\mathfrak{G}$-shtukas is to relate this stack to certain moduli spaces for local objects, called local $\mathbb{P}$-shtukas, where $\mathbb{P}$ is a parahoric group scheme. More precisely let $A_{\nu}$ be the completion of the local ring $\mathcal{O}_{C, \nu}$ at a closed point $\nu \in C$, and let $\mathbb{P}=\mathbb{P}_{\nu}:=\mathfrak{G} \times_{C}$ Spec $A_{\nu}$. We develop the theory of local $\mathbb{P}$-shtukas partly independently of global $\mathfrak{G}$-shtukas. For this purpose we replace $\operatorname{Spec} A_{\nu}$ 
by $\mathbb{D}:=$ Spec $k \llbracket z \rrbracket$ for a finite field $k$. In $[\mathrm{H}-\mathrm{V}]$ Hartl and Viehmann have introduced local $G$-shtukas for a connected reductive group $G$, as the function field analogs of $p$-divisible groups. This category has as objects pairs $\underline{\mathcal{L}}=\left(\mathcal{L}_{+}, \varphi\right)$ consisting of an $L^{+} G$-torsor $\mathcal{L}_{+}$on $S \in \mathcal{N} i l_{\mathbb{F}_{q} \llbracket \zeta \rrbracket}$ and an isomorphism of the loop torsors $\varphi: \hat{\sigma}^{*} \mathcal{L} \rightarrow \mathcal{L}$ as its objects. Here $L G$ (resp. $L^{+} G$ ) denotes the group of loops (resp. positive loops) of $G$ (see 2.3), $\mathcal{L}$ denotes the $L G$-torsor associated with $\mathcal{L}_{+}$and $\hat{\sigma}^{*} \mathcal{L}$ the pullback of $\mathcal{L}$ under the $q$-Frobenius morphism Frob $_{q}: S \rightarrow S$. For a parahoric group scheme $\mathbb{P}$ one can define local $\mathbb{P}$-shtukas in a similar way, replacing the $L^{+} G$-torsor over $S$ by $L^{+} \mathbb{P}$-torsors. We denote the resulting category by $\widehat{\mathfrak{S}} h t_{\mathbb{P}}(S)$.

Consider the formal stack $\nabla_{n} \mathscr{H}^{1}(C, \mathfrak{G})^{\underline{\nu}}$, which is obtained by taking the formal completion of the stack $\nabla_{n} \mathscr{H}^{1}(C, \mathfrak{G})$ at an $n$-tuple of characteristic places $\underline{\nu}=\left(\nu_{i}\right)$ (see section 2.1). One can extend the morphisms in the category $\nabla_{n} \mathscr{H}^{1}(C, \mathfrak{G})^{\underline{\nu}}(S)$ to quasi-isogenies (see Def 3.1.1) between global $\mathfrak{G}$-shtukas and form a category which we denote by $\mathfrak{S} h t \underline{\mathcal{L}}(S)$.

Recall that to an abelian variety $\mathcal{A}$ over $\mathbb{F}_{p}$ one can associate a $p$-divisible group $\mathcal{A}\left[p^{\infty}\right]$. In the analogous situation (see section 3.2) one can associate a tuple $\left(\underline{\mathcal{G}}_{\nu_{i}}\right)$ of local $\mathbb{P}_{\nu_{i}}$-shtuka $\underline{\mathcal{G}}_{\nu}$ to a global $\mathfrak{G}$-shtuka $\underline{\mathcal{G}}$ in $\mathfrak{S} h t t_{\mathfrak{G}}(S)$

$$
\widehat{\Gamma}: \mathfrak{S h} t_{\mathfrak{G}}^{\nu}(S) \rightarrow \prod_{\nu_{i}} \widehat{\mathfrak{S} h} t_{\mathbb{P}_{\nu_{i}}}(S) .
$$

We construct this functor in section 3.2.

In analogy with the Grothendieck-Messing theory we prove (see section 4.2.1) that the infinitesimal deformations of a global $\mathfrak{G}$-shtuka $\underline{\mathcal{G}}$ are the same as the infinitesimal deformations of the associated local $\mathbb{P}$-shtukas via the global-local functor $\widehat{\Gamma}$. Note that as we mentioned above, unlike abelian varieties, $\mathfrak{G}$-shtukas may posses more than one characteristic and we must keep track of the deformations of the local $\mathbb{P}$-shtukas at each of these characteristic places. This theorem for abelian $\tau$-sheaves (corresponding to the case $\mathfrak{G}=G l_{n}$ ) and their associated $z$-divisible groups was first stated and proved by Hartl in [Ha1].

The knowledge that the deformations of a global $\mathfrak{G}$-shtuka are ruled by the deformations of the associated local shtukas at the characteristic places looks enough enlightening to seek a similar uniformization procedure as that of Rapoport and Zink (and previously Cerednik and Drinfeld) for Shimura 
variety of PEL-type.

Let $\bar{k}$ denote an algebraic closure of the residue fields of $\nu_{i}$ for $i=$ $1, \ldots, n$. Let us set

$$
\nabla_{n} \mathscr{H}^{1}(C, \mathfrak{G})^{\underline{\underline{\nu}}}:=\nabla_{n} \mathscr{H}^{1}(C, \mathfrak{G}) \times_{C^{n}} \operatorname{Spf} \bar{k} \llbracket \underline{\zeta} \rrbracket
$$

where $\operatorname{Spf} \bar{k} \llbracket \zeta \rrbracket:=\operatorname{Spf} \bar{k} \llbracket \zeta_{1} \ldots, \zeta_{n} \rrbracket$ and $\zeta_{i}$ is a uniformizer of $\mathcal{O}_{C, \nu_{i}}$.

As Hartl and Viehmann pointed out in $[\mathrm{H}-\mathrm{V}]$ the true analogs of $\mathrm{p}$ divisible groups are "bounded" local G-shtukas. Nevertheless in section 4.1 we prove that the product $\prod_{\nu} \widehat{\mathcal{F}} l_{\mathbb{P}_{\nu_{i}}}$ can be regarded as a uniformization space for $\nabla_{n} \mathscr{H}^{1}(C, \mathfrak{G})^{\underline{\underline{\nu}}}$ already in the unbounded situation, see theorem 4.1.12. Here

$$
\widehat{\mathcal{F}}_{\mathbb{P}_{\nu_{i}}}:=\mathcal{F} \ell_{\mathbb{P}_{\nu_{i}}} \times \operatorname{Spf} \bar{k} \llbracket \zeta_{i} \rrbracket,
$$

where $\mathcal{F} \ell_{\mathbb{P}_{\nu_{i}}}$ is the local affine flag varieties corresponding to the parahoric group $\mathbb{P}_{\nu_{i}}:=\mathfrak{G} \times_{C} \operatorname{Spec} \mathcal{O}_{C, \nu_{i}}$, which is constructed by Papas and Rapoport, see $[\mathrm{PR} 2]$. Note that the ind-scheme $\widehat{\mathcal{F}}_{\mathbb{P}}$ can be viewed as a moduli space for local $\mathbb{P}$-shtukas $\underline{\mathcal{L}}$ together with a quasi-isogeny $f: \underline{\mathcal{L}} \rightarrow \underline{\mathbb{L}}_{0}$ from $\underline{\mathcal{L}}$ to a fixed trivialized local $\mathbb{P}$-shtuka $\underline{\underline{L}}_{0}$.

The analogs of the Rapoport-Zink space for local $G$-shtukas, when $G$ is a split reductive group was constructed and studied by Hartl and Viehmann in $[\mathrm{H}-\mathrm{V}]$. In chapter 6 , we generalize their construction to the case of $\mathbb{P}$-shtukas where $\mathbb{P}$ is a parahoric Bruhat-Tits group. For this purpose we introduce the notion of boundedness condition on the quasi-isogenies between local (resp. global) $\mathbb{P}$-shtukas (resp. $\mathfrak{G}$-shtukas). These bounds are essentially given by closed subschemes $\widehat{Z}$ of $\widehat{\mathcal{F}} \ell_{\mathbb{P}}$ which satisfy certain conditions, see section 6.2. Finally in this chapter we prove that the Rapoport-Zink space for local $\mathbb{P}$-shtukas $\mathcal{M}_{\mathbb{L}_{0}}^{\hat{Z}}$ is a formal scheme locally formally of finite type over $k \llbracket \zeta \rrbracket$, see 6.3.1 (also see [RZ, Thm 2.16] and [H-V, Thm 5.6]).

After the construction of the Rapoport-Zink space $\mathcal{M}_{\mathbb{L}_{0}}^{\hat{Z}}$ (for local $\mathbb{P}$ shtukas) in chapter 6 the next chapter is devoted to the construction of the uniformization map. Using the tannakian theory, we introduce the notion of the level $H$-structure (for a compact open subgroup $H \subset \mathfrak{G}\left(\mathbb{A} \frac{\nu}{Q}\right)$ ) on a global $\mathfrak{G}$-shtuka $\underline{G}$. This is done in chapter 5 . We denote by $\nabla_{n}^{H} \mathscr{H}^{1}(C, \mathfrak{G})$ the moduli stack parametrizing $\mathfrak{G}$-shtukas together with a level $H$-structure. Let $\underline{Z}:=\left(\widehat{Z}_{\nu}\right)$ be an $n$-tuple of bounds $\widehat{Z}_{\nu}$ in $\widehat{\mathcal{F}}_{\mathbb{P}_{\nu}}$. The associated bound-

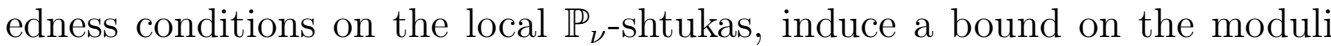
stack $\nabla_{n}^{H} \mathscr{H}^{1}(C, \mathfrak{G})^{\underline{\nu}}$. We denote the corresponding formal substack given by imposing the boundness condition $\underline{\widehat{Z}}$ by $\nabla_{n}^{H, \hat{Z}_{\underline{\nu}}} \mathscr{H}^{1}(C, \mathfrak{G})^{\underline{\nu}}$. We show in 
Proposition 6.2.18 that it is a formal algebraic stack over $\prod_{i} \operatorname{Spf} A_{\nu_{i}}$ locally of finite type. Let $\underline{\mathcal{G}}_{0}$ be a fixed global $\mathfrak{G}$-shtuka in $\nabla_{n}^{H, \hat{Z}_{\underline{\nu}}} \mathscr{H}^{1}(C, \mathfrak{G})^{\overline{\underline{\nu}}}(\bar{k})$ and set $\left(\underline{\mathcal{L}}_{\nu}\right):=\widehat{\Gamma}\left(\mathcal{G}_{0}\right)$. Let $I(Q)$ denote the group of the self quasi-isogenies of $\underline{\mathcal{G}}_{0}$. We construct the uniformization morphism

$$
\Theta: I(Q) \backslash \prod_{\nu} \mathcal{M}_{\underline{\mathcal{L}}_{\nu}}^{\hat{Z}_{\nu}} \times \mathfrak{G}\left(\mathbb{A}_{Q}^{\underline{\nu}}\right) / H \rightarrow \nabla_{n}^{H, \hat{Z}_{\underline{\nu}}} \mathscr{H}^{1}(C, \mathfrak{G})^{\underline{\underline{\nu}}}
$$

and in addition we prove that the uniformization map induces an isomorphism after passing to the completion along its image, see theorem 7.1.4. Note that the reduced subscheme of the Rapoport-Zink space for local $\mathbb{P}$ shtukas is an affine Deligne-Lusztig variety. Thus as a consequence of the uniformization theorem one can relate the rational points (of the Newton stratum) of the moduli stack of global $\mathfrak{G}$-shtukas to the rational points of certain affine Deligne-Lusztig varieties.

In the mixed characteristic set up the Schubert varieties in a twisted affine flag variety have appeared in the theory of local models for Shimura varieties. Also this anticipation is worked out for function field analogs of Shimura varieties, see [Var, Thm. 2.20] and [Dr1, Prop. 3.3]. In the last chapter we study the theory of local models for the moduli of global $\mathfrak{G}$ shtukas and briefly mention how this may counterpart with the theory of uniformzation of the moduli stack of global $\mathfrak{G}$-shtukas.

\subsection{Notation and Conventions}

$\mathbb{N}_{0} \quad$ the set of non-negative integers,

$n \in \mathbb{N}_{0} \quad$ a positive number,

$\mathbb{F}_{q} \quad$ the finite field with $q$ elements of characteristic $p$,

$C \quad$ smooth projective geometrically irreducible curve over $\mathbb{F}_{q}$,

$\nu \quad$ a closed point of $C$,

$\underline{\nu}:=\left(\nu_{i}\right) \quad$ an $n$-tuple of closed points of $C$,

$k \quad$ residue field of a place $\nu$ on $C$,

$\bar{k} \quad$ an algebraic closure of $k$,

$\hat{\sigma} \quad$ the $\sharp k$-Frobenius.

$A_{\nu} \quad$ the completion of the stalk $\mathcal{O}_{C, \nu}$ at $\nu$,

$\mathbb{A}$ the ring of integral adeles of $C$, i.e. $\overleftarrow{\mathcal{I}}_{\lim } \mathcal{O}_{C} / \mathcal{I}$ where the limit is taken over all non-zero sheaves of ideals of $\mathcal{O}_{C}$,

$\mathbb{A} \underline{\nu} \quad$ the adelic ring $\lim _{\mathcal{I}, \mathrm{V}(I) \cap\left\{\nu_{i}\right\}=\emptyset} \mathcal{O}_{C} / \mathcal{I}$ where the limit is taken over all non- 
zero sheaves of ideals of $\mathcal{O}_{C}$ whose support is disjoint from $\left\{\nu_{i}\right\}$, $\mathbb{A}_{Q}=\mathbb{A} \otimes_{\mathcal{O}_{C}} \mathbb{F}_{q}(C)$ the ring of adeles of $C$, $\mathbb{A} \frac{\nu}{Q}:=\mathbb{A} \underline{\nu} \otimes_{\mathcal{O}_{C}} \mathbb{F}_{q}(C)$, $\mathbb{D}_{R}:=$ Spec $R \llbracket z \rrbracket$ spectrum of the ring of formal power series in $z$ with coefficients in a commutative ring $R$, $\dot{\mathbb{D}}_{R}:=\operatorname{Spec} R((z))$, where $R((z)):=R \llbracket z \rrbracket\left[\frac{1}{z}\right]$, $\mathbb{D}_{n, R}:=\operatorname{Spec} R \llbracket z \rrbracket / z^{n}$, where $n \in \mathbb{N}_{0}$, $\hat{\mathbb{D}}_{R}:=\operatorname{Spf} R \llbracket z \rrbracket$ formal spectrum of $R \llbracket z \rrbracket$ When $R=k$ we drop the subscript $R$ from the notation of $\mathbb{D}_{R}, \dot{\mathbb{D}}_{R}, \mathbb{D}_{n, R}$ and $\hat{\mathbb{D}}_{R}$.

$\zeta \quad$ an indeterminate over $\mathbb{F}_{q}$,

$\mathfrak{G}$ a smooth affine group scheme over $C$,

$G$ a reductive group over $\dot{\mathbb{D}}$,

$\mathbb{P}$ a smooth affine group scheme of finite type over $\mathbb{D}$,

$P_{\eta} \quad$ generic fibre of $\mathbb{P}$,

$\mathbb{P}_{\nu} \quad$ the group scheme $\mathfrak{G}_{\nu}:=\mathfrak{G} \times_{C} \mathcal{O}_{C, \nu}$ over $\operatorname{Spec} \mathcal{O}_{C, \nu}$,

$\hat{\mathbb{P}}_{\nu} \quad$ pull-back of $\mathbb{P}_{\nu}$ under the map $\operatorname{Spf} A_{\nu} \rightarrow \operatorname{Spec} \mathcal{O}_{C, \nu}$.

Let $S$ be a scheme. We denote by $\sigma_{S}: S \rightarrow S$ its Frobenius endomorphism which acts as the $q$-power map on the structure sheaf,

$C_{S}=C \times_{\mathbb{F}_{q}} S$, when $S=\operatorname{Spec} R$ we also denote $C_{R}:=C \times_{\mathbb{F}_{q}} S$, $\sigma=i d_{C} \times \sigma_{S}$.

For any facet $a$ in the Bruhat- Tits building of $G$ over $\dot{\mathbb{D}}, \mathbb{P}_{a}$, denotes the unique smooth group scheme, with connected fibers, over $\mathbb{D}$, such that $\mathbb{P}_{a}(k \llbracket z \rrbracket)$ is equal to the parahoric subgroup of $G(K)$ attached to $a$, cf. [BT2, 1.7].

Let $\widehat{S}$ be a formal scheme. We denote by $\mathcal{N} i l p \widehat{S}$ the category of schemes over $\widehat{S}$ on which an ideal of definition of $\widehat{S}$ is locally nilpotent, equiped with the étale topology.

Let $H$ be a sheaf of groups over $X$, we let $\mathscr{H}^{1}(X, H)$ denote the category fibered in groupoids over the category of schemes $S c h$, such that the objects over $S, \mathscr{H}^{1}(X, H)(S)$, are $H$-torsors over $X \times_{\mathbb{Z}} S$ and morphisms are isomorphisms of $H$-torsors. 


\section{Chapter 2}

\section{Glob. and Loc. Shtukas, Def. and Their Moduli Stacks}

\subsection{Formal Algebraic Stacks}

Recall that a formal space $\widehat{X}$ over a formal scheme $\widehat{S}$ is a sheaf of sets on the site $\mathcal{N} i l p_{\widehat{S}}$. In addition it is called a formal algebraic space if the diagonal morphism $\widehat{X} \rightarrow \widehat{X} \times \widehat{S} \widehat{X}$ is representable by a quasicompact morphism of formal schemes and there is a formal scheme $\widehat{X}^{\prime}$ over $\widehat{S}$ and a morphism of formal $\widehat{S}$-spaces $\widehat{X}^{\prime} \rightarrow \widehat{X}$ which is representable by an étale surjective morphism of formal schemes.

Let $\mathcal{X}$ be a stack over a scheme $S$. Let $S_{0}$ be a locally closed subscheme of $S$. Let $\widehat{S}$ denote the formal completion of $S$ along $S_{0}$. Restricting the fibered functor $\mathcal{X}$ to the category $\mathcal{N} i p_{\widehat{S}}$ gives a category $\widehat{\mathcal{X}}$ fibred in groupoids over $\mathcal{N}_{i l} p_{\widehat{S}}$ which inherits the following properties from $\mathcal{X}$

i) for every $V$ in $\mathcal{N} i l p_{\widehat{S}}$ and $x, y$ in $\widehat{\mathcal{X}}(V)$ the presheaf

$$
\begin{aligned}
\text { Isom }: S c h / V & \rightarrow \text { Sets } \\
U \rightarrow V & \longmapsto \operatorname{Hom}_{\widehat{\mathcal{X}}(U)}\left(x_{U}, y_{U}\right),
\end{aligned}
$$

is a sheaf on $S c h / V$.

ii) for every covering $V_{i} \rightarrow V$ in $\mathcal{N} i l p_{\widehat{S}}$ all descent data for this covering are effective.

Further more if $\mathcal{X}$ is an algebraic stack (resp. of DM-type) we have 
(a) the diagonal 1-morphism $\widehat{\mathcal{X}} \rightarrow \widehat{\mathcal{X}} \times \widehat{\widehat{S}} \widehat{\mathcal{X}}$ over $\widehat{S}$ is representable (i.e. the fiber over any $U$-valued point, $\mathcal{U} \in \mathcal{N} i l p_{\widehat{S}}$ is representable by a formal algebraic $\widehat{S}$-space), separated, and quasi-compact,

(b) there exists a formal algebraic $\widehat{S}$-space $\widehat{X}$ and a presentation

$$
P: \widehat{X} \rightarrow \widehat{\mathcal{X}}
$$

of formal $\widehat{S}$-stacks which is representable by a smooth (étale) and surjective morphism of formal algebraic $\widehat{S}$-spaces.

Abstractifying the above easy observation, we phrase that in the following way

Definition 2.1.1. A category $\widehat{\mathcal{X}}$ fibered in groupoids over $\mathcal{N}_{i l p} \widehat{S}$ is called a formal stack if it has the properties i) and ii) indicated above. Also we say $\widehat{\mathcal{X}}$ is formal algebraic stack if in addition it is subject to a) and b) above. A formal algebraic stack $\widehat{\mathcal{X}}$ is called Deligne-Mumford (or of DM-type) if the presentation $P$ can be chosen étale.

Example 2.1.2. Again mimicking the idea behind the construction of quotient stacks one can define the quotient of a formal space by a formal group scheme.

Let $\widehat{T}$ be a formal $\widehat{S}$-scheme and let $\widehat{G}$ be a formal $\widehat{T}$-group space (i.e. a group object in the category of formal $\widehat{T}$-spaces). A (right) $\widehat{G}$-torsor is a formal $\widehat{T}$-space $\widehat{\mathcal{P}}$ with an action of $\widehat{G}$ (from the right) such that there is a covering $\widehat{T}^{\prime} \rightarrow \widehat{T}$ over $\widehat{S}$ for which $\widehat{\mathcal{P}} \times_{\widehat{T}} \widehat{T}^{\prime}$ is $\widehat{G} \times_{\widehat{T}} \widehat{T}^{\prime}$-isomorph to $\widehat{G} \times_{\widehat{T}} \widehat{T}^{\prime}$ which acts on itself by right translation.

Let $\widehat{X}$ be a formal $\widehat{S}$-space, $\widehat{Y}$ an $\widehat{X}$-space (i.e. a formal $\widehat{S}$-space equipped with a morphism $\widehat{Y} \rightarrow \widehat{X}$ ) and $\widehat{G}$ an $\widehat{X}$-group space which acts on $\widehat{Y}$ from the right. We define the quotient stack $[\widehat{Y} / \widehat{G}]$ as the following category fibered in groupoids over the category of formal $\widehat{S}$-schemes:

For every formal $\widehat{S}$-scheme $\widehat{T}$ the category $[\widehat{Y} / \widehat{G}](\widehat{T})$ consists of all triples $(x, \widehat{\mathcal{P}}, \alpha)$ where $x \in \widehat{X}(\widehat{T}), \widehat{\mathcal{P}}$ is a $\widehat{G} \times_{\widehat{X}, x} \widehat{T}$-torsor and $\alpha: \widehat{\mathcal{P}} \rightarrow \widehat{Y} \times_{\widehat{X}, x} \widehat{T}$ is a $\widehat{G} \times{ }_{\widehat{X}, x} \widehat{T}$-equivariant morphism of formal $\widehat{T}$-spaces. One easily verifies that the quotient $[\widehat{Y} / \widehat{G}]$ is a formal $\widehat{S}$-stack.

Note that when $\widehat{G}$ is smooth, the natural morphism $\widehat{Y} \rightarrow[\widehat{Y} / \widehat{G}]$ is a smooth presentation and therefore $[\widehat{Y} / \widehat{G}]$ is a formal algebraic stack. Moreover if $\widehat{G}$ is étale over $\widehat{X}$ then $[\widehat{Y} / \widehat{G}]$ is a formal Deligne-Mumford stack. 
Let $S_{\text {red }}$ denote the underlying reduced subscheme of $\widehat{S}$. In a similar way, as that of formal schemes, one has a functor

$$
-_{\text {red }}: \widehat{\mathfrak{S t a}} / \widehat{S} \rightarrow \mathfrak{S t a} / S_{\text {red }}
$$

where $\mathfrak{S t a}$ (resp. $\widehat{\mathfrak{S t a}}$ ) denotes the 2-category of algebraic stacks (resp. formal algebraic stacks) over $S_{\text {red }}($ resp. $\widehat{S})$. Namely, let $\widehat{\mathcal{X}}$ be a formal algebraic $\widehat{S}$-stack and let $P: \widehat{X} \rightarrow \widehat{\mathcal{X}}$ be a presentation. We define the underlying reduced stack $\mathcal{X}=\widehat{\mathcal{X}}_{\text {red }}$ as follows:

For every $U$ in $\mathcal{N} i l p_{\widehat{S}}, \mathcal{X}(U)$ is the full subcategory of $\widehat{\mathcal{X}}(U)$ whose objects are the $x \in \mathcal{X}(U)$ such that there is a covering $U^{\prime} \rightarrow U$ in $\mathcal{N} i l p_{\widehat{S}}$, an element $x^{\prime} \in \widehat{X}_{\text {red }}\left(U^{\prime}\right)$, and an isomorphism in $\widehat{\mathcal{X}}\left(U^{\prime}\right)$ between $x_{U^{\prime}}$ and $P\left(x^{\prime}\right)$. The above functor restricts to a functor from the category of formal algebraic stacks of DM-type to algebraic stacks of DM-type.

Similarly one can define the notion of quasi-coherent sheaves on a formal algebraic stack $\widehat{\mathcal{X}}$. We say that a sheaf of ideals $\mathcal{I}$ of $\mathcal{O}_{\widehat{\mathcal{X}}}$ is an ideal of definition of $\widehat{\mathcal{X}}$ if for any presentation $P: \widehat{X} \rightarrow \widehat{\mathcal{X}}$ the ideal sheaf $P^{*} \mathcal{I}$ is an ideal of definition for $\widehat{X}$.

We say that $\widehat{\mathcal{X}}$ is locally noetherian if the presentation $\widehat{X}$ is locally noetherian. If $\widehat{\mathcal{X}}$ is locally noetherian then it posses a unique largest ideal of definition, namely the ideal defining the closed sub-stack $\mathcal{X}_{\text {red }}$. We denote this ideal by $\mathcal{I}_{\widehat{\mathcal{X}}}$.

From now on we assume that all formal algebraic stacks are locally noetherian.

Definition 2.1.3. Let $\widehat{S}$ be the completion of a scheme $S$ along a closed sub-scheme $S_{0}$. Let $\mathcal{X}$ be an algebraic $S$-stack and $\mathcal{Z}$ a locally closed substack of $\mathcal{X}$, contained in $\mathcal{X} \times_{S} S_{0}$. We define the formal completion $\widehat{\mathcal{X}}_{/ \mathcal{Z}}$ as the full sub-category of $\mathcal{X}$ consisting of those points $p$ of $\mathcal{X}(U)$ such that the $U_{\text {red-point }}$ associated to $p$ be a $U_{\text {red }}$-point of $\mathcal{Z}$.

Proposition 2.1.4. The formal completion $\widehat{\mathcal{X}}_{/ \mathcal{Z}}$ of $\widehat{\mathcal{X}}$ along $\mathcal{Z}$ is a formal algebraic $\widehat{S}$-stack. Moreover if $\mathcal{X}$ is an algebraic S-stack of DM-type then $\widehat{\mathcal{X}}_{/ \mathcal{Z}}$ is a formal algebraic $\widehat{S}$-stack of DM-type. If $\mathcal{I}$ is the ideal sheaf on $\mathcal{X}$ defining $\mathcal{Z}$ then $\widehat{\mathcal{X}}_{/ \mathcal{Z}}$ is $\mathcal{I}$. $\mathcal{O}_{\widehat{\mathcal{X}}_{/ \mathcal{Z}}}$-adic.

Proof. cf. [Ha1, Appendix Prop A.14].

Definition 2.1.5. A 1-morphism $f: \widehat{\mathcal{Y}} \rightarrow \widehat{\mathcal{X}}$ of formal algebraic stacks is called adic if $f^{*} \mathcal{I}_{\widehat{\mathcal{X}}}$ is an ideal of definition for $\widehat{\mathcal{Y}}$ 
Remark 2.1.6. If $\widehat{X}$ is an adic formal algebraic $\widehat{S}$-space and $H$ a finite étale $\widehat{S}$-group scheme then the quotient $[\widehat{X} / H]$ is an adic formal algebraic $\widehat{S}$-stack of DM-type. In this case, the canonical projection $\widehat{X} \rightarrow[\widehat{X} / H]$ is an étale presentation for $[\widehat{X} / H]$.

\subsection{Global $\mathfrak{G}$-Shtukas}

Let $\mathfrak{G}$ be a smooth affine group scheme on the curve $C$ over $k$.

We denote by $\mathscr{H}^{1}(C, \mathfrak{G})$ the moduli stack of $\mathfrak{G}$-bundles on $C$. The following is well-known:

Remark 2.2.1. The stack $\mathscr{H}^{1}(X, \mathfrak{G})$ is a smooth algebraic $k$-stack, which is locally of finite type, see theorem 6.2.12.

For $c$ in the set of the connected components $\pi_{0}\left(\mathscr{H}^{1}(X, \mathfrak{G})\right)$, we denote by $\mathscr{H}^{1}(X, \mathfrak{G})_{c}$ the corresponding open substack.

Definition 2.2.2. Let $\mathfrak{G}$ be a smooth affine group scheme on a curve $C$ over $k$. We say that $\mathfrak{G}$ is a parahoric group scheme over $C$ if

(a) all geometric fibers of $\mathfrak{G}$ are connected and the generic fiber $\mathfrak{G}_{\eta}$ of $\mathfrak{G}$ is reductive over $k(C)$,

(b) for any ramification point $\nu$ of $\mathfrak{G}$ (i.e. those points $\nu$ of $C$, such that the fiber above $\nu$ is not reductive) the group scheme $\mathbb{P}_{\nu}:=\mathfrak{G}_{\nu}$ is a parahoric group scheme over $\mathcal{O}_{C, \nu}$, as defined by Bruhat-Tits (cf. [BT1, Définition 5.2.6]).

Let us mention that when $\mathfrak{G}$ is parahoric over $C$, Pappas and Rapoport [PR3] conjectured that there is a canonical isomorphism

$$
\pi_{0}\left(\mathscr{H}^{1}(C, \mathfrak{G})\right) \cong \pi_{1}\left(\mathfrak{G}_{\eta}\right)_{\Gamma} .
$$

Where $\pi_{1}\left(\mathfrak{G}_{\eta}\right)$ denotes the fundamental group of $\mathfrak{G}_{\eta}$ and the right hand side are the co-invariants under $\Gamma:=\operatorname{Gal}\left(k(C)^{\text {sep }} / k(C)\right)$.

The following proposition of J. Heinloth [He, Thm. 6] affirms their anticipation to some extent. 
Proposition 2.2.3. Let $\mathfrak{G}$ be a parahoric Bruhat-Tits group scheme over $C$ with semi-simple generic fiber, then

$$
\pi_{0}\left(\mathscr{H}^{1}(C, \mathfrak{G})\right)=\pi_{1}\left(\mathfrak{G}_{\eta}\right)_{\Gamma} .
$$

For a divisor $D$ on $C$ let $\mathscr{H}_{D}^{1}(C, \mathfrak{G})$ denote the stack classifying $\mathfrak{G}$ bundles with $D$-level structure (i.e. a section of the $\mathfrak{G}$-bundle over $D \times_{\mathbb{F}_{q}} S$ ), or equivalently define $\mathscr{H}_{D}^{1}(C, \mathfrak{G})$ as a category fibred in groupoids, which assigns to a scheme $S$ over $k$ the category whose objects are

$$
\mathcal{O} b \mathscr{H}_{D}^{1}(C, \mathfrak{G})(S):=\left\{(\mathcal{G}, \psi) ; \mathcal{G} \in \mathscr{H}^{1}(C, \mathfrak{G}), \psi: \mathcal{G}_{\mid D \times S} \stackrel{\sim}{\longrightarrow} \mathfrak{G} \times_{C} D \times_{\mathbb{F}_{q}} S\right\},
$$

and its morphisms are those isomorphisms of $\mathfrak{G}$-bundles that preserve the $D$-level structure.

Definition-Remark 2.2.4. Consider the following functor

$$
\begin{aligned}
S \longmapsto & \left\{\left(\mathcal{G}, s_{1} \ldots, s_{n}, \varphi\right) ; \mathcal{G} \in \mathcal{O} b \mathscr{H}^{1}(C, \mathfrak{G})(S), s_{i} \in C(S)\right. \\
& \left.\varphi:\left.\mathcal{G}\right|_{C_{S} \backslash \cup_{i} \Gamma_{s_{i}}} \rightarrow \mathfrak{G} \times_{C} C_{S} \backslash \cup_{i} \Gamma_{s_{i}} \text { is a trivializaition }\right\} .
\end{aligned}
$$

This functor is representable by an ind-scheme $G r_{\mathfrak{B}, n}$ over $C^{n}$, locally of finite type, see [Ga, Proposition 2.2.3]. The ind-scheme $G r_{\mathfrak{G}, n}$ is called the global affine grassmannian.

Definition 2.2.5. For each natural number $n$, let $H_{e c k} \mathfrak{G , D , n}$ be the stack whose $S$ valued points are triples

i) $(\mathcal{G}, \psi)$ and $\left(\mathcal{G}^{\prime}, \psi^{\prime}\right)$ in $\mathscr{H}_{D}^{1}(C, \mathfrak{G})(S)$,

ii) sections $c_{1}, \ldots c_{n} \in(C \backslash D)(S)$

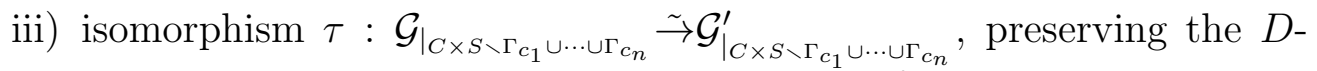
level structures. Here $\Gamma_{c_{i}}$ denotes the graph of the section $c_{i}$.

Note that forgetting the isomorphism $\tau$ defines a morphism

$$
\text { Hecke }_{\mathfrak{G}, D, n} \rightarrow \mathscr{H}_{D}^{1}(C, \mathfrak{G}) \times \mathscr{H}_{D}^{1}(C, \mathfrak{G}) \times(C \backslash D)^{n} .
$$

We denote by $p r_{1}$ and $p r_{2}$ the projections, respectively to the first and second factor.

Remark 2.2.6. We will show in proposition 6.2.16 that for a parahoric group $\mathfrak{G}$ the stack $H e c k e_{\mathfrak{G}, D, n}$ can be viewed as a locally closed substack of a $G r_{G l_{r}}$-fibration over $\mathscr{H}^{1}(C, \mathfrak{G})$. 
Definition 2.2.7. Assume that we have two morphisms $f, g: X \rightarrow Y$ of schemes (stacks), we denote by $\operatorname{ker}(f, g: X \rightrightarrows Y)$ the pull back of the diagonal under the morphism $(f, g): X \rightarrow Y \times Y$.

Definition 2.2.8. We define the moduli stack $\nabla_{n} \mathscr{H}_{D}^{1}(C, \mathfrak{G})$ to be the preimage in $H_{e c k} e_{\mathfrak{G}, D, n}$ of the graph of the Frobenius morphism. In other words

$$
\nabla_{n} \mathscr{H}_{D}^{1}(C, \mathfrak{G})=\operatorname{ker}\left(p r_{1}, \sigma_{\mathscr{H}_{D}^{1}(C, \mathfrak{G})} \circ p r_{2}: \operatorname{Hecke}_{\mathfrak{G}, D, n} \rightrightarrows \mathscr{H}_{D}^{1}(C, \mathfrak{G})\right) .
$$

We call this the moduli stack of global $\mathfrak{G}$-shtukas.

Each object $\underline{\mathcal{G}}$ in $\operatorname{Ob}\left(\nabla_{n} \mathscr{H}_{D}^{1}(C, \mathfrak{G})(S)\right)$ is called a global $\mathfrak{G}$-shtuka over $S$ and the corresponding sections $\left(c_{1}, \ldots, c_{n}\right)$ are called the characteristic sections (or simply characteristics) of $\underline{\mathcal{G}}$.

More explicitly a global $\mathfrak{G}$-shtuka $\underline{\mathcal{G}}$ over $S$ in $S c h / \mathbb{F}_{q}$ is a tuple $\left(\mathcal{G}, s_{1}, \ldots, s_{n}, \varphi\right)$ consisting of a $\mathfrak{G}$-bundle $\mathcal{G}$ over $C_{S}$, an $n$-tuple of (characteristic) sections $\left(s_{1}, \ldots, s_{n}\right) \in C^{n}(S)$ and an isomorphism $\varphi:\left.\left.\sigma^{*} \mathcal{G}\right|_{C_{S} \backslash \cup_{i} \Gamma_{s_{i}}} \sim \mathcal{\rightarrow} \mathcal{G}\right|_{C_{S} \backslash \cup_{i} \Gamma_{s_{i}}}$.

We will drop the subscript $n$ from the notation of $\nabla_{n} \mathscr{H}^{1}(C, \mathfrak{G}), G r_{\mathfrak{G}, n}$,

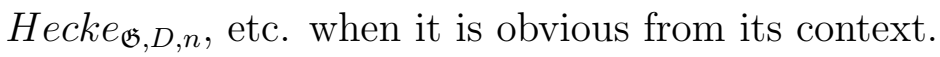

\subsection{Loop groups and Local $\mathbb{P}$-Shtukas}

Let $\mathbb{X}$ be a scheme over $\mathbb{D}$. Consider the following functor

$$
\begin{aligned}
L^{+}-: S c h / \mathbb{D} & \rightarrow \operatorname{PreShv} / k \\
\mathbb{X} & \longmapsto \underline{L^{+} \mathbb{X}}: \operatorname{Spec} R \mapsto \mathbb{X}\left(\mathbb{D}_{R}\right),
\end{aligned}
$$

from the category of schemes over $\mathbb{D}$ to the category of pre-sheaves on $S c h / k$. After restrict the above functor to the category of affine schemes of finite type over $\mathbb{D}$, one can easily see that the resulting functor factors through the full subcategory of schemes over $k$. We denote the scheme representing the functor $L^{+} \mathbb{X}$ by $L^{+} \mathbb{X}$ and we call it the space of positive loops on $\mathbb{X}$.

Similarly we define the following functor

$$
\begin{aligned}
L-: S c h / \dot{\mathbb{D}} & \rightarrow \operatorname{PreShv/k} \\
X & \longmapsto \underline{L X}: \operatorname{Spec} R \mapsto X\left(\dot{\mathbb{D}}_{R}\right) .
\end{aligned}
$$


When we restrict the above functor to the category of affine $\dot{\mathbb{D}}$-schemes of finite type, then this functor factors through the full sub category of indschemes over $k$. We call the ind-scheme $L X$ (which represents the functor $\underline{L X}$ ) the loop space of $X$.

Remark 2.3.1. Let $\mathbb{X}$ be a scheme of finite type over $\mathbb{D}$. If $\mathbb{X}$ is formally smooth (resp. reduced, connected), then so is $L^{+} \mathbb{X}$.

Remark 2.3.2. Let $X$ be a scheme of finite type over $\dot{\mathbb{D}}$. If $X$ is smooth, then $L X$ is also formally smooth. However the functor $X \mapsto L X$ may no longer preserve connectedness and reducedness. For instance one can verify that for $X:=\mathbb{G}_{m}$ the space of loops $L \mathbb{G}_{m}$ is neither connected nor reduced.

For the details and functorial properties of these constructions see [PR2].

Let $G($ resp. $\mathbb{P}$ ) be a linear algebraic group over $\dot{\mathbb{D}}$ (resp. flat affine group scheme of finite type over $\mathbb{D}$ ). The group of loops (resp. positive loops) associated to $G$ (resp. $\mathbb{P}$ ) is the ind-group scheme (resp. affine group scheme) $L G\left(\right.$ resp. $\left.L^{+} \mathbb{P}\right)$ over Spec $k$.

Let $\eta$ be the generic point of $\mathbb{D}$ and $P_{\eta}$ be the generic fibre of $\mathbb{P}$. Let $\mathscr{H}^{1}\left(\operatorname{Spec} k, L^{+} \mathbb{P}\right)\left(\right.$ resp. $\left.\mathscr{H}^{1}\left(\operatorname{Spec} k, L P_{\eta}\right)\right)$ denote the classifying space of

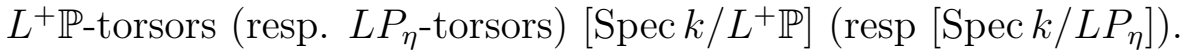

Consider the natural 1-morphism

$$
\mathscr{H}^{1}\left(\operatorname{Spec} k, L^{+} \mathbb{P}\right) \rightarrow \mathscr{H}^{1}\left(\operatorname{Spec} k, L P_{\eta}\right), \mathcal{L}_{+} \mapsto \mathcal{L}
$$

of categories fibred in groupoids.

Let us denote the restriction of $\mathscr{H}^{1}\left(\operatorname{Spec} k, L^{+} \mathbb{P}\right)\left(\right.$ resp. $\left.\mathscr{H}^{1}\left(\operatorname{Spec} k, L P_{\eta}\right)\right)$ to the site $\mathcal{N} i l p_{k \llbracket \zeta \rrbracket}$ still with the same notation. We define the space of local shtukas with $\mathbb{P}$-structure as follows.

Definition 2.3.3. Let $\mathcal{C}$ be the fiber product

$$
\mathscr{H}^{1}\left(\operatorname{Spec} k, L^{+} \mathbb{P}\right) \times \mathscr{H}^{1}\left(\operatorname{Spec} k, L P_{\eta}\right) \mathscr{H}^{1}\left(\operatorname{Spec} k, L^{+} \mathbb{P}\right)
$$

of groupoids. Let $p r_{i}$ denote the projections to the i-th factor. We define the groupoid of local $\mathbb{P}$-shtukas $S h t t_{\mathbb{P}}^{\hat{\mathbb{P}}}$ to be

$$
\operatorname{ker}\left(p r_{1}, \hat{\sigma} \circ p r_{2}: C \rightrightarrows \mathscr{H}^{1}\left(\operatorname{Spec} k, L^{+} \mathbb{P}\right)\right) \text {. }
$$

We call an object of the category $S h t \hat{\mathbb{P}}(S)$ a local shtuka with $\mathbb{P}$-structure over $S$ (or simply local $\mathbb{P}$-shtuka over $S$ ). 
20CHAPTER 2. GLOB. AND LOC. SHTUKAS, DEF. AND THEIR MODULI STACKS

More explicitly the category $S h t t_{\mathbb{P}}^{\hat{\mathbb{P}}}(S)$ has as objects pairs $\underline{\mathcal{L}}=\left(\mathcal{L}_{+}, \varphi\right)$ consisting of an $L^{+} \mathbb{P}$-torsor $\mathcal{L}_{+}$on $S \in \mathcal{N} i l p_{\hat{\mathbb{D}}}$ and an isomorphism of the loop torsors $\varphi: \hat{\sigma}^{*} \mathcal{L} \rightarrow \mathcal{L}$ as its objects.

Definition 2.3.4. A local $\mathbb{P}$-shtuka $\left(\mathcal{L}_{+}, \varphi\right)$ is called étale if $\varphi$ comes from an isomorphism of $L^{+} \mathbb{P}$-torsors $\hat{\sigma}^{*} \mathcal{L}_{+} \rightarrow \mathcal{L}_{+}$. 


\section{Chapter 3}

\section{A starting point of the analogy}

\subsection{Rigidity of quasi-isogenies}

Recall that (for a prime number $p$ ) a $(p$-)quasi-isogeny between abelian varieties $\mathscr{A}_{1}$ and $\mathscr{A}_{2}$ can be defined as a roof

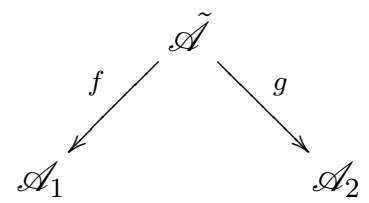

of isogenies of abelian varieties (such that a power of $p$ kills the kernel of $f$ and $g$ ).

This inspires to establish the following analogous definition for a quasiisogeny between global $\mathfrak{G}$-shtukas.

Definition 3.1.1. A quasi-isogeny between global $\mathfrak{G}$-shtukas

$$
\underline{\mathcal{G}}:=(\mathcal{G}, \tau) \rightarrow \underline{\mathcal{G}}^{\prime}:=\left(\mathcal{G}^{\prime}, \tau^{\prime}\right)
$$

with the same characteristics, is an isomorphism $\left.\left.\mathcal{G}\right|_{C_{S} \backslash D_{S}} \rightarrow \mathcal{G}^{\prime}\right|_{C_{S} \backslash D_{S}}$, where $D$ is an effective divisor on $C$, such that $\tau^{\prime} \sigma^{*}(f)=f \tau$. We denote the category of global $\mathfrak{G}$-shtukas over $S$ (with exactly $n$ characteristic sections), with quasi-isogenies as its morphisms, by $\mathfrak{S} h t_{\mathfrak{G}}^{n}(S)$ (when the number of the characteristic sections is clear from the context we drop the superscript $n$ ).

Spelling out the observation we recalled above for " $p$-divisible groups", we analogously define 
Definition 3.1.2. A quasi-isogeny between local $\mathbb{P}$-shtukas

$$
\underline{\mathcal{L}}:=\left(\mathcal{L}_{+}, \varphi\right) \rightarrow \underline{\mathcal{L}}^{\prime}:=\left(\mathcal{L}_{+}^{\prime}, \varphi^{\prime}\right)
$$

over $S$ is an isomorphism of the associated $L P_{\eta^{-}}$torsors $f: \mathcal{L} \rightarrow \mathcal{L}^{\prime}$ such that the following diagram

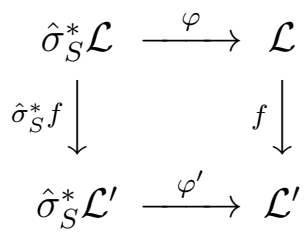

commutes.

We denote by $\operatorname{QIsog}_{S}\left(\underline{\mathcal{L}}, \underline{\mathcal{L}}^{\prime}\right)$ the set of quasi-isogenies between $\underline{\mathcal{L}}$ and $\underline{\mathcal{L}}^{\prime}$ over $S$. We denote the category of local $\mathbb{P}$-shtukas over $S$, with quasi-isogenies as its morphisms, by $\widehat{\mathfrak{S} h}_{\mathbb{P}}(S)$.

Let $\bar{S}$ be a closed subscheme of $S$, defined by a locally nilpotent sheaf of ideals. Let $X$ and $Y$ be $p$-divisible groups over $S$. Further assume that $p$ is locally nilpotent on $S$. Then a quasi-isogeny between $X_{\bar{S}}$ and $Y_{\bar{S}}$ lifts uniquely over $S$. This phenomenon was first observed by Drinfeld, see [Dr2].

The analogy between $p$-divisible groups and local shtukas, we mentioned above, develops further, and in particular, the following proposition states that quasi-isogenies between local $\mathbb{P}$-shtukas enjoy the mentioned rigidity property, namely a quasi-isogeny between local $\mathbb{P}$-shtukas lifts over infinitesimal thickenings, thanks to the Frobenius connections.

This interesting feature of shtukas first mentioned in [Ha1] for abelian sheaves and $z$-divisible groups and later in $[\mathrm{H}-\mathrm{V}]$ for $G$-shtukas, where $G$ is a constant reductive group over $\mathbb{F}_{q}$. Their observation and proof is obviously valid replacing $G$-shtukas by $\mathbb{P}$-shtukas.

Proposition 3.1.3. (Rigidity of quasi-isogenies) Let $S$ be a scheme in $\mathcal{N}$ ilp $k \llbracket \zeta \rrbracket$ and let $i: \bar{S} \rightarrow S$ be a closed immersion defined by a sheaf of ideals $\mathcal{I}$ which is locally nilpotent. Let $\underline{\mathcal{L}}=\left(\mathcal{L}_{+}, \varphi\right)$ and $\underline{\mathcal{L}}^{\prime}=\left(\mathcal{L}_{+}^{\prime}, \varphi^{\prime}\right)$ be two shtukas in $\widehat{\mathfrak{S h}} t_{\mathbb{P}}(S)$. Then

$$
Q I \operatorname{sog}_{S}\left(\underline{\mathcal{L}}, \underline{\mathcal{L}}^{\prime}\right) \longrightarrow Q I \operatorname{sog}_{S}\left(i^{*} \underline{\mathcal{L}}, i^{*} \underline{\mathcal{L}}^{\prime}\right), f \mapsto i^{*} f
$$

is a bijection of sets.

Proof. Arguing by induction over $\mathcal{O}_{S} / \mathcal{I}^{q^{n}}$ it suffices to treat the case where $\mathcal{I}^{q}=(0)$. In this case the $q$-Frobenius factors as $S \stackrel{j}{\rightarrow} \bar{S} \stackrel{i}{\rightarrow} S$ where $j$ is the 
identity on the underlying topological space $|\bar{S}|=|S|$ and on the structure sheaf this factorization is given by

$$
\begin{array}{rlcll}
\mathcal{O}_{S} & \stackrel{i^{*}}{\longrightarrow} & \mathcal{O}_{\bar{S}} & \stackrel{j^{*}}{\longrightarrow} & \mathcal{O}_{S} \\
b & \mapsto & b \bmod \mathcal{I} & \mapsto & b^{q} .
\end{array}
$$

Therefore $\sigma^{*} f=j^{*}\left(i^{*} f\right)$ for any $f \in \operatorname{QIsog}_{S}\left(\underline{\mathcal{L}}, \underline{\mathcal{L}}^{\prime}\right)$. We obtain the diagram

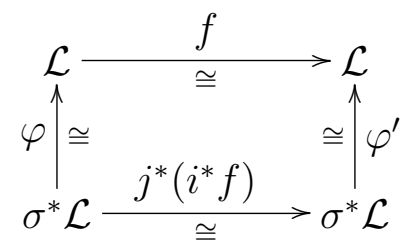

from which the bijectivity is obvious.

Remark 3.1.4. By similar arguments as above one may see that quasiisogenies between global $\mathfrak{G}$-shtukas also enjoy similar rigidity property.

\subsection{The Global-Local Functor}

Denote by $\mathscr{A} \mathscr{V}_{S}$ the category whose objects are abelian schemes over some base $S$ over a field of characteristic $p$, with quasi-isogenies as its morphisms. Let $\mathscr{B} \mathscr{T}_{S}$ denote the category whose objects are $p$-divisible groups and morphisms are the quasi-isogenies between them.

Recall that assigning the $p$-divisible group $A\left[p^{\infty}\right]$ (i.e. the inductive system formed by the $p^{n}$-torsion subgroup schemes $A\left[p^{n}\right]$ ) to an abelian scheme $A$ in $\mathscr{A} \mathscr{V}_{S}$ defines a functor

$$
\underline{\Gamma}: \mathscr{A}^{\mathscr{V}_{S}} \rightarrow \mathscr{B} \mathscr{T}_{S}
$$

In the sequel of this section we investigate the analogous functor which goes from the category of global $\mathfrak{G}$-shtukas to the category of local $\mathbb{P}$ shtukas.

Definition 3.2.1. Let $\hat{\mathbb{P}}$ be the formal group scheme over $\hat{\mathbb{D}}$, obtained by the formal completion of $\mathbb{P}$ along $V(z)$. A formal $\hat{\mathbb{P}}$-torsor over a $k$-scheme $S$ is a $z$-adic formal scheme $\hat{\mathcal{P}}$ over $\hat{\mathbb{D}} \times{ }_{\text {Spf } k} S$ together with an action $\hat{\mathbb{P}} \times_{\hat{\mathbb{D}}} \hat{\mathcal{P}} \rightarrow \hat{\mathcal{P}}$ of $\hat{\mathbb{P}}$ on $\hat{\mathcal{P}}$ such that there is a covering $\hat{\mathbb{D}}_{S^{\prime}}:=\hat{\mathbb{D}} \times_{\text {Spf } k} S^{\prime} \rightarrow \hat{\mathbb{D}}_{S}:=\hat{\mathbb{D}} \times_{\text {Spf } k} S$ where $S^{\prime} \rightarrow S$ is an fppf-covering and a $\hat{\mathbb{P}}$-equivariant isomorphism 


$$
\hat{\mathcal{P}} \hat{\times}_{\hat{\mathbb{D}}_{S}} \hat{\mathbb{D}}_{S^{\prime}} \rightarrow \hat{\mathbb{P}}_{\hat{\mathbb{D}}_{\hat{\mathbb{D}}}} \hat{\mathbb{D}}_{S^{\prime}}
$$

Here $\hat{\mathbb{P}}$ acts on itself by right multiplication.

Let $\mathscr{H}^{1}(\hat{\mathbb{D}}, \hat{\mathbb{P}})$ be the category fibered in groupoids that assigns to each

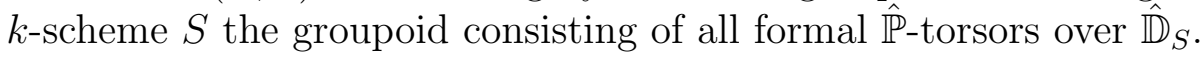

In [H-V, Proposition 2.2.a)] Hartl and Viehmann prove that for a split reductive group $G$, there is a bijection of (pointed) sets between the Čech cohomology $\check{\mathrm{H}}^{1}\left(S_{f p q c}, L^{+} G\right)$ and the set of isomorphism classes of z-adic formal schemes over $\hat{\mathbb{D}} \times_{\operatorname{Spf} k} S$. Following their arguments one can even see that there is a natural isomorphism between the corresponding categories.

Proposition 3.2.2. There is a natural isomorphism

$$
\mathscr{H}^{1}(\hat{\mathbb{D}}, \hat{\mathbb{P}}) \stackrel{\sim}{\rightarrow} \mathscr{H}^{1}\left(\operatorname{Spec} k, L^{+} \mathbb{P}\right)
$$

of groupoids.

Proof. To a given element $\hat{\mathcal{P}}$ of $\mathscr{H}^{1}(\hat{\mathbb{D}}, \hat{\mathbb{P}})(S)$ one can associate the following sheaf

$$
\begin{aligned}
\underline{\mathcal{K}}:(\text { fppf/S }) & \rightarrow \text { Sets } \\
T & \mapsto \operatorname{Hom}_{\hat{\mathbb{D}}_{S}}\left(\hat{\mathbb{D}}_{T}, \hat{\mathcal{P}}\right),
\end{aligned}
$$

where $(f p p f / S)$ denotes the big $f p p f$-site on $S$. This sheaf is precisely a principal homogeneous space under the action of $L^{+} \mathbb{P}(T)=\operatorname{Hom}_{\hat{\mathbb{D}}}\left(\hat{\mathbb{D}}_{T}, \hat{\mathbb{P}}\right)$. Since the group of positive loops of $\mathbb{P}$ is affine this functor can be represented by a $L^{+} \mathbb{P}$-torsor $\mathcal{K}$.

Let $\mathcal{K}$ be a $L^{+} \mathbb{P}$-torsor. Let $S^{\prime} \rightarrow S$ be a covering that trivialises $\mathcal{K}$ and fix $\mathcal{K}_{S^{\prime}} \stackrel{\sim}{\rightarrow} L^{+} \mathbb{P} \times S^{\prime}$. This gives a 1-cocycle $g \in L^{+} \mathbb{P}\left(S^{\prime \prime}\right)$, where $S^{\prime \prime}=S^{\prime} \times{ }_{S} S^{\prime}$. Now $\bar{g}=g\left(\bmod z^{n}\right)$ can be viewed as descent data on $\hat{\mathbb{P}} \times_{\mathbb{D}} \mathbb{D}_{n, S^{\prime}}=\mathbb{P} \times_{\mathbb{D}} \mathbb{D}_{n, S^{\prime}}$. Since $\mathbb{D}_{n, S^{\prime}} \rightarrow \mathbb{D}_{n, S}$ is an $f p p f$-cover and $\mathbb{P}$ is affine, the descent data is effective by [BLR, $\S 6.1$, Theorem 6] and gives an affine finitely presented smooth scheme $\hat{\mathcal{G}}_{n}$ over $\mathbb{D}_{n, S}$ by $\left[\right.$ EGA, IV $\mathrm{IV}_{2}$, Proposition 2.7 .1 and $\mathrm{IV}_{4}$, Corollaire 17.7.3]. These schemes form an inductive system $\left\{\hat{\mathcal{G}}_{n}\right\}_{n \in \mathbb{N}}$. Now set $\hat{\mathcal{G}}:=\underset{n}{\lim } \hat{\mathcal{G}}_{n}$, the existence of this limit (in the category of $z$-adic formal schemes over $\hat{\mathbb{D}}_{S}$ ) follows from [EGA, $\mathrm{I}_{\text {new }}$, Corollary 10.6.4]. This shows that the functor is essentially surjective. By the above construction we see that the functor is also fully faithful. 
Let $\nu$ be a place on $C$. Let $\hat{\mathbb{P}}_{\nu}$ denote the formal group scheme obtained by restricting $\mathfrak{G}$ to the formal spectrum $\operatorname{Spf} A_{\nu}$, note that $\operatorname{Spf} A_{\nu} \cong \hat{\mathbb{D}} \hat{\otimes} \kappa(\nu)$. Assume that we have a section $s: S \rightarrow C$ and furthermore we know that this section factors through $\operatorname{Spf} A_{\nu}$. In this case we have

$$
\operatorname{Spf} A_{\nu} \hat{\otimes} S \cong \coprod_{i} \mathrm{~V}\left(\mathfrak{a}_{\nu, i}\right) \cong \coprod_{i \in \mathbb{Z} /[\kappa(\nu): k]} \hat{\mathbb{D}}_{S},
$$

where $\mathrm{V}\left(\mathfrak{a}_{\nu, i}\right)$ denotes the component identified by the ideal

$$
\mathfrak{a}_{\nu, i}=\left\langle a \otimes 1-1 \otimes s^{*}(a)^{q^{i}} ; a \in A_{\nu}\right\rangle .
$$

Remark 3.2.3. Note that $\sigma$ cyclically permutes these components and thus $\sigma^{\operatorname{deg} \nu}$ leaves each of the components $\mathrm{V}\left(\mathfrak{a}_{\nu, i}\right)$ stable.

Let $\left(C \times \mathscr{H}^{1}(C, \mathfrak{G})\right)^{\widehat{v}}$ be the formal stack given by taking completion of $C \times \mathscr{H}^{1}(C, \mathfrak{G})$ along $\nu \in C$, see chapter 2.1. Consider the following functor

$$
\left(C \times \mathscr{H}^{1}(C, \mathfrak{G})\right)^{\widehat{\nu}}(S) \rightarrow \mathscr{H}^{1}\left(\operatorname{Spec} k, L^{+} \mathbb{P}\right)(S),
$$

which sends $(s, \mathcal{G})$ to the $L^{+} \mathbb{P}_{\nu}$-torsor associated to the $\hat{\mathbb{P}}_{\nu}$-torsor $\hat{\mathcal{P}}_{\nu}$ given by the connected component of $\mathcal{G} \times_{C_{S}, s} \operatorname{Spf} \mathcal{O}_{C, v} \hat{\otimes} S$ lying over $\mathrm{V}\left(\mathfrak{a}_{\nu, 0}\right)$, according to proposition 3.2.2.

Fix a tuple $\underline{\nu}:=\left(\nu_{i}\right)_{i=1 \ldots n}$ of places on $C$. Let the formal stack $\nabla \mathscr{H}^{1}(C, \mathfrak{G})^{\underline{\nu}}$ be the completion of the algebraic stack $\nabla \mathscr{H}^{1}(C, \mathfrak{G})$ along $\underline{\nu} \in C^{n}$.

We define the category $\mathfrak{S} h t \underline{\mathcal{G}}(S)$ to be the category whose objects are the same as $\nabla \mathscr{H}^{1}(C, G)^{\underline{\nu}}(S)$, and the morphisms are the quasi-isogenies between them. The functor 3.2.2 induces the following functor

$$
\widehat{\Gamma}: \mathfrak{S h} t_{\mathfrak{G}}^{\nu}(S) \rightarrow \prod_{\nu_{i}} \widehat{\mathfrak{S} h} t_{\mathbb{P}_{\nu_{i}}}(S),
$$

which sends $(\mathcal{G}, \tau)$ to $\left(\left(\hat{\mathcal{P}}_{\nu_{i}}, \hat{\tau}_{\nu_{i}}^{\text {deg }\left(\nu_{i}\right)}\right)\right)$, see also remark 3.2.3. We call this functor the global-local functor.

Remark 3.2.4. Note that in a similar way one can associate a local $\mathbb{P}_{\nu^{-}}$ shtuka $\underline{\mathcal{G}}_{\nu}$ to a global $\mathfrak{G}$-shtuka $\underline{\mathcal{G}}$ at a place $\nu$ outside the characteristic places $\nu_{i}$. Note that the main difference is that there is no distinguished component given by the characteristic section, see [BH, Prop 2.5]. 


\section{Chapter 4}

\section{Unbounded Uniformization and The Deformation Theory}

\subsection{Construction of the Uniformization map}

Let us first recall a well-known theorem, proved by A. Beauville and Y. Laszlo, that enables one to glue quasi-coherent sheaves along infinitesimal neighbourhoods.

Let $X$ be a scheme and $D$ be an effective Cartier divisor on $X$. We let $\mathfrak{Q} \mathfrak{C o h}(X)\left(\right.$ resp. $\left.\mathfrak{Q} \mathfrak{C}_{0} h_{D}(X)\right)$ denote the category of quasi-coherent $\mathcal{O}_{X^{-}}$ modules ( resp. quasi-coherent $\mathcal{O}_{X}$-modules that have no nonzero sections supported on $D)$.

Theorem 4.1.1. Let $\pi: X^{\prime} \rightarrow X$ be a morphism of schemes. Consider the following diagram

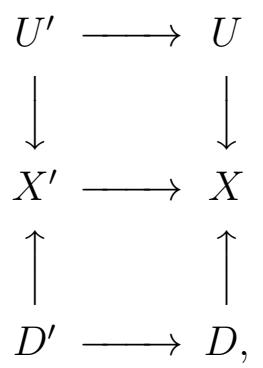

where $D$ is an effective Cartier divisor on $X$ and $D^{\prime}$ its pull back under $\pi$, $U$ and $U^{\prime}$ are respectively the complement of $D$ and $D^{\prime}$. Now if $D^{\prime} \cong D$ under $\pi$, then the induced diagram 


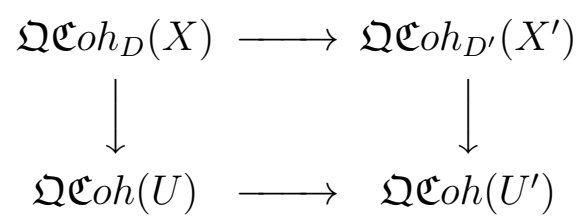

is a Cartesian diagram of categories.

Proof. This is a reformulation of the well-known patching theorem of Beauville and Laszlo, cf. [BL]. Note however that they consider the case that $X^{\prime}$ is the completion of $X$ along $D$, but what they actually need for the proof is that $D^{\prime}$ maps isomorphically to $D$ under $\pi$.

Definition 4.1.2. Let $\mathbb{P}$ be a flat affine group scheme of finite type over $\mathbb{D}$ and $P_{\eta}$ be the generic fibre as before. Let $\underline{\mathcal{F}}_{\mathbb{P}}$ be the fpqc-sheaf, associated to the presheaf

$$
R \mapsto \mathbb{P}(R((z))) / \mathbb{P}(R \llbracket z \rrbracket),
$$

on the category of $k$-algebras.

Proposition 4.1.3. Let $\mathbb{P}$ be as in the above definition. Then

a) there is a faithful representation $\mathbb{P} \rightarrow G L_{n}$ over $\mathbb{D}$, for some $n$.

b) There exists a closed immersion of group schemes $\mathbb{P} \rightarrow G L_{n} \times \mathbb{G}_{m}$ such that the quotient fppf-sheaf is representable by a quasi-affine scheme over $\mathbb{D}$.

Proof. cf. [PR2, Proposition 1.3].

Remark 4.1.4. When $\mathbb{P}=\mathrm{GL}_{n}$, the fpqc-sheaf $\mathcal{F} \ell_{\mathbb{P}}$ is called the affine Grassmanian. The $R$-valued points of the affine Grassmanian parametrize lattices $\mathfrak{L}$ in $R((z))^{n}$, i.e. $R \llbracket z \rrbracket$-submodules which are locally free of rank $n$ on $\operatorname{Spec} R$ and $\mathfrak{L} \otimes R((z))=R((z))^{n}$. It is well-known that the affine Grassmannian is representable by an ind-k-scheme which is ind-proper over $k$ and the quotient morphism $L \mathrm{GL}_{n} \rightarrow L \mathrm{GL}_{n} / L^{+} G L_{n}=\mathcal{F} \ell_{\mathrm{GL}_{n}}$ admits a section locally for the étale topology, see proposition 2.3 and theorem 2.5 of $[\mathrm{BL} 1]$.

The following theorem of Pappas and Rapoport states a partial generalization of the mentioned properties of affine Grassmannian, excluding the properness of the twisted affine flag variety $\mathcal{F} \ell_{\mathbb{P}}$. Note however that proposition 6.2.7 establishes the ind-properness of the twisted affine flag varieties in certain cases. 
Proposition 4.1.5. Assume that $\mathbb{P}$ is smooth over $\mathbb{D}$. Then the fpqc-sheaf $\underline{\mathcal{F}}_{\mathbb{P}}$ is represented by an ind-k-scheme $\mathcal{F} \ell_{\mathbb{P}}$ of ind-finite type over $k$. The quotient morphism $L P_{\eta} \rightarrow \mathcal{F} \ell_{\mathbb{P}}$ admits sections locally for the étale topology, i.e. $L^{+} \mathbb{P}$-equivariant isomorphisms

$$
\operatorname{Spec}(R) \times_{\mathcal{F} \ell_{\mathbb{P}}} L P_{\eta} \cong \operatorname{Spec}(R) \times_{\operatorname{Spec}(k)} L^{+} \mathbb{P},
$$

for each point of $\mathcal{F} \ell_{\mathbb{P}}$ with values in a strictly henselian ring $R$.

Proof. cf. [PR2, thm 1.4].

Let $k^{\prime}$ be a finite extension of $k$. For $S$ in $\mathcal{N}_{i l p} p_{k^{\prime} \llbracket \zeta \rrbracket}$ let $\bar{S}$ denote the closed subscheme $\mathrm{V}(\zeta) \subseteq S$.

Definition 4.1.6. To a given local $\mathbb{P}$-shtuka $\underline{\mathcal{L}}_{0}$ over $\bar{S} \in S c h / \mathbb{F}_{q}$ we associate the following functor

$$
\begin{aligned}
& \underline{\mathcal{M}}_{\underline{\mathcal{L}}_{0}}: \mathcal{N} i p_{\bar{S} \times_{\mathbb{F}_{q}} \operatorname{Spf} \mathbb{F}_{q} \llbracket \zeta \rrbracket} \rightarrow \text { Sets } \\
& T \mapsto\{(\underline{\mathcal{L}}, \bar{\delta}) ; \underline{\mathcal{L}} \text { is a local } \mathbb{P} \text {-shtuka over } T \text { and } \\
& \left.\bar{\delta}: \underline{\mathcal{L}}_{\bar{T}} \rightarrow \underline{\mathcal{L}}_{0, \bar{T}} \text { is a quasi-isogeny over } \bar{T}\right\} / \sim .
\end{aligned}
$$

Here $(\underline{\mathcal{L}}, \bar{\delta}) \sim\left(\underline{\mathcal{L}}^{\prime}, \bar{\delta}^{\prime}\right)$ if $\bar{\delta}^{-1} \circ \bar{\delta}^{\prime}$ lifts to an isomorphism $\underline{\mathcal{L}}^{\prime} \rightarrow \underline{\mathcal{L}}$.

Remark 4.1.7. Note that by rigidity of quasi-isogenies the functor $\underline{\mathcal{M}}_{\underline{\mathcal{L}}_{0}}$ is naturally isomorphic to the functor

$T \mapsto\{$ Isomorphy class of $(\underline{\mathcal{L}}, \delta) ; \underline{\mathcal{L}}$ is a local $\mathbb{P}$-shtuka over $T$ and $\delta: \underline{\mathcal{L}}_{T} \rightarrow \underline{\mathcal{L}}_{0, T}$ is a quasi-isogeny $\}$.

Now let us view the formal scheme Spf $k \llbracket \zeta \rrbracket$ as an ind-scheme

$$
\underset{n}{\lim } \operatorname{Spec} k[\zeta] /\left(\zeta^{n}\right) .
$$

We may form the fiber product $\mathcal{F} \ell_{\mathbb{P}} \times_{k} \operatorname{Spf} k \llbracket \zeta \rrbracket$ in the category of indschemes (see [BD, 7.11.1]). Note that this fiber product can be either viewed as the restriction of the sheaf $\mathcal{F} \ell_{\mathbb{P}}$ to the $f p p f$-site of schemes in $\mathcal{N} i l p_{k \llbracket \zeta \rrbracket}$ or also as the formal completion of $\mathcal{F} \ell \times{ }_{k}$ Spec $k \llbracket \zeta \rrbracket$ along the special fiber $\mathrm{V}(\zeta) \subset \mathcal{F} \ell_{\mathbb{P}} \times_{k}$ Spec $k \llbracket \zeta \rrbracket$. 
Consider a local $\mathbb{P}$-shtuka $\underline{\mathbb{L}}$ over $k \llbracket \zeta \rrbracket$. Since $L^{+} \mathbb{P}$ is smooth (see remark 2.3.1), $\underline{\mathbb{L}}$ admits a trivialization over a separable extension $k^{\prime} / k$. So we trivialize $\underline{\mathbb{L}}_{k} \otimes_{k \llbracket \zeta \rrbracket} k^{\prime} \llbracket \zeta \rrbracket \cong\left(L^{+} \mathbb{P}, b \hat{\sigma}^{*}\right)$, for some $b \in L P_{\eta}\left(k^{\prime} \llbracket \zeta \rrbracket\right)$ and set $\underline{\mathbb{L}}_{0}:=\left(L^{+} \mathbb{P}, b \hat{\sigma}^{*}\right)$. Let $\bar{b} \in L P_{\eta}\left(k^{\prime}\right)$ denote the reduction of $b$ modulo $\zeta$.

The following observation was first recorded in $[\mathrm{H}-\mathrm{V}]$ for $G$-shtukas, where $G$ is a (constant) split reductive group over $\mathbb{F}_{q}$. Their proof (with a small modification, thanks to proposition 4.1.5 above of Pappas and Rapoport) works for the general case.

Proposition 4.1.8. The ind-scheme $\mathcal{F} \ell_{\mathbb{P}} \times_{k}$ Spf $k^{\prime} \llbracket \zeta \rrbracket$ pro-represents the functor

$$
\underline{\mathcal{M}}_{\mathbb{L}_{0}}: \mathcal{N} i l p_{k \llbracket \zeta \rrbracket} \longrightarrow \text { Sets } .
$$

Proof. Let us fix the notation $\underline{\mathcal{M}}:=\underline{\mathcal{M}}_{\underline{\mathbb{L}}_{0}}$ and $\widehat{\mathcal{F}} \ell_{k^{\prime}}=\mathcal{F} \ell_{\mathbb{P}} \times_{k}$ Spf $k^{\prime} \llbracket \zeta \rrbracket$. Note that we may regard $\underline{\mathcal{M}}$ as the equivalent form mentioned in remark 4.1.7.

Consider a pair $(\underline{\mathcal{L}}, \delta)=\left(\left(\mathcal{L}_{+}, \varphi\right), \delta\right) \in \mathcal{M}(S)$. Choose an étale covering $S^{\prime} \rightarrow S$ which trivializes $\underline{\mathcal{L}}$, so the quasi isogeny $\delta$ is given by an element $g^{\prime} \in$ $L P_{\eta}\left(S^{\prime}\right)$. The image of the element $g^{\prime} \in L P_{\eta}\left(S^{\prime}\right)$ in $\widehat{\mathcal{F}}_{k^{\prime}}\left(S^{\prime}\right)$ is independent of the choice of trivialization, and since $\underline{\mathcal{L}}$ is defined over $S$, it descends to a point $x \in \widehat{\mathcal{F}}_{k^{\prime}}(S)$.

Note in particular that $\varphi_{S^{\prime}}$ is determined by $b$ and $g^{\prime}$

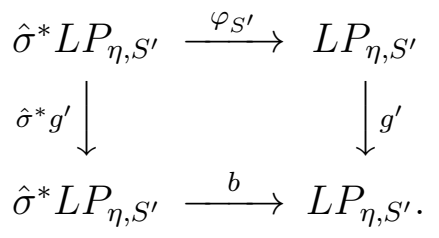

Conversely let $x \in \widehat{\mathcal{F}}_{k^{\prime}}(S)$ for a scheme $S$ in $\mathcal{N} i l p_{k^{\prime} \llbracket \zeta \rrbracket}$. The projection morphism $L P_{\eta} \rightarrow \mathcal{F} \ell$ admits local sections for the étale topology by proposition 4.1.5. Consequently there is an étale covering $S^{\prime} \rightarrow S$ such that $x$ is represented by an element $g^{\prime} \in L G\left(S^{\prime}\right)$. Define $\left(\mathcal{L}_{+}^{\prime}, \varphi^{\prime}, \delta^{\prime}\right)$ over $S^{\prime}$ as follows. Let $\mathcal{L}^{\prime}=L^{+} \mathbb{P}_{S^{\prime}}$, let the quasi-isogeny $\delta^{\prime}:\left(\mathcal{L}_{+}^{\prime}, \varphi^{\prime}\right) \rightarrow \underline{\mathbb{L}}_{0, S^{\prime}}$ be given by $y \mapsto g^{\prime} y$, and the Frobenius by $\varphi^{\prime}=\left(g^{\prime}\right)^{-1} b \sigma^{*}\left(g^{\prime}\right) \sigma^{*}$. We descend $\left(\mathcal{L}_{+}^{\prime}, \varphi^{\prime}, \delta^{\prime}\right)$ to $S$. For an $S$-scheme $Y$ let $Y^{\prime}=Y \times_{S} S^{\prime}$ and $Y^{\prime \prime}=Y^{\prime} \times_{Y} Y^{\prime}$, and let $p_{i}: Y^{\prime \prime} \rightarrow Y^{\prime}$ be the projection onto the $i$-th factor. Since $g^{\prime}$ comes from an element $x \in \widehat{\mathcal{F}}_{k^{\prime}}(S)$ there is an $h \in L^{+} \mathbb{P}\left(S^{\prime \prime}\right)$ with $p_{1}^{*}\left(g^{\prime}\right)=p_{2}^{*}\left(g^{\prime}\right) \cdot h$. Consider the fpqc-sheaf $\mathcal{L}_{+}$on $S$ whose sections over an $S$-scheme $Y$ are 
given by

$$
\mathcal{L}_{+}(Y):=\left\{y^{\prime} \in L^{+} \mathbb{P}\left(Y^{\prime}\right): p_{1}^{*}\left(y^{\prime}\right)=h^{-1} \cdot p_{2}^{*}\left(y^{\prime}\right) \text { in } L^{+} \mathbb{P}\left(Y^{\prime \prime}\right)\right\}
$$

on which $L^{+} \mathbb{P}(Y)$ acts by right multiplication. Then $\mathcal{L}$ is a $L^{+} \mathbb{P}$-torsor on $S$ because over $Y=S^{\prime}$ there is a trivialization

$$
L^{+} \mathbb{P}_{S^{\prime}} \stackrel{\sim}{\longrightarrow} \mathcal{L}_{+S^{\prime}}, \quad f \mapsto h p_{1}^{*}(f) \in L^{+} \mathbb{P}\left(S^{\prime \prime}\right)
$$

due to the cocycle condition on $h$. Moreover, $\varphi^{\prime}$ descends to an isomorphism

$$
\varphi: \sigma^{*} \mathcal{L}(Y) \stackrel{\mathcal{L}}{\longrightarrow}(Y), \sigma^{*}\left(y^{\prime}\right) \mapsto\left(g^{\prime}\right)^{-1} b \sigma^{*}\left(g^{\prime}\right) \sigma^{*}\left(y^{\prime}\right)
$$

making $\left(\mathcal{L}_{+}, \varphi\right)$ a local $\mathbb{P}$-shtuka over $S$. Also $\delta^{\prime}$ descends to a quasi-isogeny of local $\mathbb{P}$-shtukas

$$
\begin{aligned}
\delta: \mathcal{L}(Y) & \stackrel{L}{\longrightarrow} P_{\eta}(Y)=\left\{f^{\prime} \in L P_{\eta}\left(Y^{\prime}\right): p_{1}^{*}\left(f^{\prime}\right)=p_{2}^{*}\left(f^{\prime}\right) \text { in } L G\left(Y^{\prime \prime}\right)\right\} \\
y^{\prime} & \mapsto g^{\prime} y^{\prime} .
\end{aligned}
$$

Note that this is well defined. Namely, if $g^{\prime}$ is replaced by $\tilde{g}^{\prime}$ with $u^{\prime}=$ $\left(\tilde{g}^{\prime}\right)^{-1} g^{\prime} \in L^{+} \mathbb{P}\left(S^{\prime}\right)$ then left multiplication with $u^{\prime}$ defines an isomorphism

$$
\left(L^{+} \mathbb{P}_{S^{\prime}},\left(g^{\prime}\right)^{-1} b \sigma^{*}\left(g^{\prime}\right) \sigma^{*}, g^{\prime}\right) \stackrel{\sim}{\longrightarrow}\left(L^{+} \mathbb{P}_{S^{\prime}},\left(\tilde{g}^{\prime}\right)^{-1} b \sigma^{*}\left(\tilde{g}^{\prime}\right) \sigma^{*}, \tilde{g}^{\prime}\right) .
$$

Also $\tilde{h}=p_{2}^{*}\left(u^{\prime}\right) h p_{1}^{*}\left(u^{\prime}\right)^{-1}$ and hence left multiplication with $u^{\prime}$ descends to an isomorphism $\underline{\mathcal{L}} \stackrel{\sim}{\longrightarrow} \widetilde{\mathcal{L}}$ over $S$.

Remark 4.1.9. For any $\widehat{\mathcal{P}}$ in $\mathscr{H}^{1}(\hat{\mathbb{D}}, \hat{\mathbb{P}})(R)$ one can find an étale covering $R \rightarrow R^{\prime}$ such that $\widehat{\mathcal{P}}$ maps to an object in the isomorphy class of the trivial element in $\mathscr{H}^{1}(\hat{\mathbb{D}}, \hat{\mathbb{P}})\left(R^{\prime}\right)$. Indeed, we may assume that the restriction of $\widehat{\mathcal{P}}$ to $V(z) \subseteq \hat{\mathbb{D}}_{R}$ has a section and then by smoothness this section extends over $\hat{\mathbb{D}}_{R}$.

Remark 4.1.10. Let $S=$ Spec $R$ be an affine scheme and $s: S \rightarrow C$ an $S$-valued point of $C$. We denote by ( ) the restriction morphism

$$
\mathscr{H}^{1}\left(C_{R}, \mathfrak{G}\right) \rightarrow \mathscr{H}^{1}\left(C_{R} \backslash \Gamma_{s}, \mathfrak{G}\right) .
$$

Now suppose that the morphism $s: S \rightarrow C$ factors through $\operatorname{Spf} A_{\nu} \rightarrow C$.

The completion ${\widehat{\left(C_{S}\right)}}_{\Gamma_{s}}$ of the relative curve $C_{S}$ along the graph $\Gamma_{s}$ of the characteristic section $s$ equals the formal spectrum of the completion 
of $A_{\nu, R}:=A_{\nu} \otimes R$ with respect to the ideal $\mathcal{I}_{\Gamma_{s}}$ of $\Gamma_{s}$. Let us denote the corresponding ring by $\hat{B}$. We have

$$
\hat{B}:=\prod_{\vartheta \in \operatorname{Gal}\left(\kappa(\nu) / \mathbb{F}_{q}\right)} R \llbracket z_{\vartheta}-\zeta_{\vartheta} \rrbracket \cong \prod_{\vartheta \in \operatorname{Gal}\left(\kappa(\nu) / \mathbb{F}_{q}\right)} R \llbracket z_{\vartheta} \rrbracket,
$$

see the discussion before constructing the morphism 3.2.2.

We denote by $\mathbb{D}\left(\Gamma_{s}\right)\left(\right.$ resp. $\left.\hat{\mathbb{D}}\left(\Gamma_{s}\right)\right)$ the component of Spec $\hat{B}($ resp. Spf $\hat{B})$ given by the section $s$. Define $\dot{\mathbb{D}}\left(\Gamma_{s}\right):=\mathbb{D}\left(\Gamma_{s}\right) \otimes_{A_{\nu, R}} D\left(\Gamma_{s}\right)$, where $D\left(\Gamma_{s}\right):=$ $\operatorname{Spec} A_{\nu, R} \backslash \Gamma_{s}$. Note that there is a (non canonic) isomorphism $\dot{\mathbb{D}}\left(\Gamma_{s}\right) \cong$ Spec $R((z))$. So we fix an isomorphism $\dot{\mathbb{D}}\left(\Gamma_{s}\right) \cong \dot{\mathbb{D}}_{R}$. Assigning the following $L P_{\eta^{-}}$-torsor

$$
R^{\prime} \mapsto \dot{\mathcal{G}}\left(R^{\prime} \hat{\otimes}_{R} \mathbb{D}\left(\Gamma_{s}\right) \otimes_{\mathcal{O}_{C_{R}}} \dot{\mathbb{D}}\left(\Gamma_{s}\right)\right) \hat{=} \dot{\mathcal{G}}\left(\dot{\mathbb{D}}_{R^{\prime}}\right)
$$

to a $\mathfrak{G}$-bundle $\dot{\mathcal{G}}$ over $C_{R} \backslash \Gamma_{s}$, defines the following 1-morphism

$$
()_{\eta}: \mathscr{H}^{1}\left(C_{R} \backslash \Gamma_{s}, \mathfrak{G}\right) \rightarrow \mathscr{H}^{1}\left(R, L P_{\eta}\right)
$$

of groupoids.

Recall that to a section $s$ as in the above, one can also associate the following 1-morphism

$$
\widehat{(~)}_{s}: \mathscr{H}^{1}\left(C_{R}, \mathfrak{G}\right) \rightarrow \mathscr{H}^{1}\left(R, L^{+} \mathbb{P}_{\nu}\right),
$$

see morphism 3.2.2.

Definition 4.1.11. For a local $\mathbb{P}$-shtuka $\underline{\mathcal{L}}_{0}$ over $\bar{S}$ we denote by $J_{\mathcal{L}}$ the group of self quasi-isogenies $Q I \operatorname{sog}_{\bar{S}}\left(\underline{\mathcal{L}}_{0}, \underline{\mathcal{L}}_{0}\right)$ of $\underline{\mathcal{L}}_{0}$.

Note that by the definition of the space $\underline{\mathcal{M}}_{\underline{\mathcal{L}}_{0}}$, there is a natural action of the group $J_{\underline{\mathcal{L}}_{0}}$ on $\underline{\mathcal{M}}_{\underline{\mathcal{L}}_{0}}$.

In the mixed characteristic set up the Schubert varieties in affine Grassmaniann has appeared as the local model for the integral models of Shimura varieties with parahoric level structure. The similar fact is also worked out for function field analogs for Shimura varieties, see [Var] (and also chapter 8 of this thesis). We will return to this interesting discussion in the last chapter, nevertheless, as we shall see in the next theorem, the local affine flag varieties can also appear as the uniformization space for the function field analogs for Shimura varieties with parahoric level structure. 
Consider the tuple $\underline{\nu}=\left(\nu_{1}, \ldots, \nu_{n}\right)$, where $\nu_{i}$ is a place on $C$. Let $\zeta_{i}$ denote a uniformizer of $C$ at the place $\nu_{i}$ and define

$$
\nabla_{n} \mathscr{H}^{1}(C, \mathfrak{G})^{\overline{\underline{\nu}}}:=\nabla_{n} \mathscr{H}^{1}(C, F G) \times_{C^{n}} \operatorname{Spf} \bar{k} \llbracket \underline{\zeta} \rrbracket,
$$

where $\bar{k} \llbracket \zeta \rrbracket:=\bar{k} \llbracket \zeta_{1}, \ldots, \zeta_{n} \rrbracket$. Note that we may view the formal stack $\nabla_{n} \mathscr{H}^{1}(C, \mathfrak{G})^{\overline{\underline{\nu}}}$ as an ind-algebraic stack over $\bar{k} \llbracket \zeta \rrbracket:=\bar{k} \llbracket \zeta_{1}, \ldots, \zeta_{n} \rrbracket$ (see proposition 6.2.18).

The following theorem can be regarded as unbounded uniformization of the completion $\nabla_{n} \mathscr{H}^{1}(C, \mathfrak{G})^{\underline{\underline{\nu}}}$ of the moduli stack of global $\mathfrak{G}$-shtukas $\nabla_{n} \mathscr{H}^{1}(C, \mathfrak{G})$ at $\underline{\nu}=\left(\nu_{1}, \ldots, \nu_{n}\right)$.

Theorem 4.1.12. Let $\underline{\mathcal{G}}_{0}$ be a global $\mathfrak{G}$-shtuka in $\mathfrak{S} h t_{\mathfrak{G}}(\bar{S})$ for $\bar{S} \in S c h / \mathbb{F}_{q}$. Let $\left(\underline{\mathcal{L}}_{\nu_{i}}\right)_{i}:=\widehat{\Gamma}\left(\underline{\mathcal{G}}_{0}\right)$ (recall that the functor $\underline{\widehat{\Gamma}}$ was defined in 3.2.3). Then there is a natural transformation

$$
\Psi\left(\underline{\mathcal{G}}_{0}\right): \prod_{\nu_{i}} \underline{\mathcal{M}}_{\underline{\mathcal{L}}_{\nu_{i}}} \rightarrow \nabla_{n} \mathscr{H}^{1}(C, \mathfrak{G})^{\underline{\underline{\nu}}} \times_{\mathbb{F}_{q}} \bar{S} .
$$

In particular when $S=$ Spec $k$ this gives the following morphism of formal stacks

$$
\Psi:=\Psi\left(\underline{\mathcal{G}}_{0}\right): \prod_{\nu_{i}} \widehat{\mathcal{F}} \ell_{\mathbb{P}_{\nu_{i}}} \rightarrow \nabla_{n} \mathscr{H}^{1}(C, \mathfrak{G})^{\overline{\underline{\nu}}},
$$

where $\widehat{\mathcal{F}} l_{\mathbb{P}_{\nu_{i}}}:=\mathcal{F} \ell_{\mathbb{P}_{\nu_{i}}} \times_{\kappa\left(\nu_{i}\right)}$ Spf $A_{\nu_{i}}$. In addition the last morphism is ind-proper and formally étale over its image.

Proof. Let $\nu$ be a place of $C$. Let $R$ be an object of $\mathcal{N}_{i l p}$ and let $s$ : Spec $R \rightarrow C$ be the induced map. Let $G:=P_{\eta}$ denote the generic fiber of $\mathbb{P}_{\nu}$.

As a consequence of the theorem 4.1.1, applied to the situation that $X^{\prime}=$ $\mathbb{D}\left(\Gamma_{s}\right), D=\Gamma_{s}$ and $\pi$ is the obvious morphism $\mathbb{D}\left(\Gamma_{s}\right) \rightarrow C_{S}$, we have the following Cartesian diagram

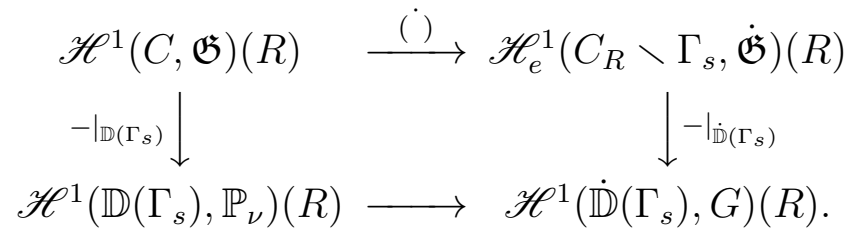

of groupoids. Here $\mathscr{H}_{e}^{1}\left(C_{R} \backslash \Gamma_{s}, \dot{\mathfrak{G}}\right)(R)$ is the full subcategory of $\mathscr{H}^{1}\left(C_{R} \backslash\right.$ $\left.\Gamma_{s},\left.\mathfrak{G}\right|_{\dot{C}}\right)(R)$ consisting of those $G$-torsors over $C_{S} \backslash \Gamma_{s}$ that can be extended 
to a $\mathfrak{G}$-torsor over the whole relative curve $C_{S}$. The above diagram induces the following diagram of groupoids

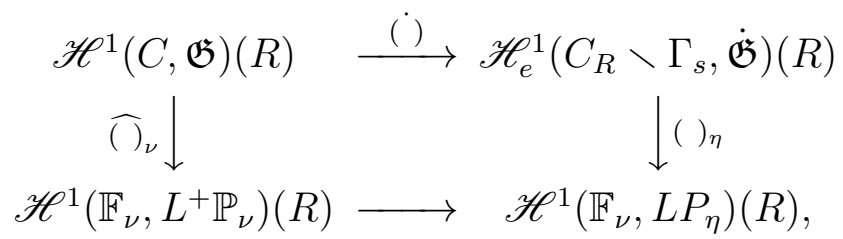

see remark 4.1.10 for the morphisms in this diagram. We claim that this diagram is cartesian. Note that by remark 2.2.1 and remark 4.1 .9 we may reduce to the case that $R$ is noetherian. In this case one can easily check that the functor

$$
\mathscr{H}^{1}\left(\mathbb{D}\left(\Gamma_{s}\right), \mathbb{P}_{\nu}\right)(R) \rightarrow \mathscr{H}^{1}\left(\hat{\mathbb{D}}\left(\Gamma_{s}\right), \hat{\mathbb{P}}_{\nu}\right)(R) \cong \mathscr{H}^{1}\left(\operatorname{Spec} k, L^{+} \mathbb{P}\right)(R)
$$

(resp. $\left.\quad \mathscr{H}^{1}\left(\mathbb{D}\left(\Gamma_{s}\right), \mathbb{P}_{\nu}\right)(R) \rightarrow \mathscr{H}^{1}\left(\mathbb{F}_{\nu}, L G\right)(R)\right)$ is an equivalence of categories (resp. fully faithful) and thus we may conclude that the above diagram is cartesian.

We may equivalently view the functor $\underline{\mathcal{M}}_{\underline{\mathcal{L}}_{\nu_{i}}}$ as the functor mentioned in remark 4.1.7.

Let $T \stackrel{\iota}{\rightarrow} S$ be an $S$-scheme and take an element $\left(\left(\underline{\mathcal{L}}_{\nu_{i}}^{\prime}\right), \hat{f}_{i}\right)_{i}$ of $\prod_{\nu_{i}} \underline{\mathcal{M}}_{\underline{\mathcal{L}}_{\nu_{i}}}(T)$ (in the sense of remark 4.1 .7$)$. Let us set $\underline{\mathcal{G}}_{0}:=\left(\mathcal{G}_{0}, \tau_{0}\right)$. Let $(\dot{\mathcal{G}}, \hat{\mathcal{G}}, \varphi)$ be the triple associated with the $\mathfrak{G}$-bundle $\mathcal{G}_{0}$, regarding the above cartesian diagram for the section $\nu_{1}$. Now the triple $\left(\dot{\mathcal{G}}_{0, T}, \mathcal{L}_{\nu_{1}}^{\prime}, \hat{f}^{-1} \varphi_{T}\right)$ defines a $\mathfrak{G}$ bundle over $C_{T}$ which by construction inherits a Frobenius automorphism $\tau_{1}$ over $C_{T} \backslash \Gamma_{s} \times_{S} T$ from $\iota^{*} \underline{\mathcal{G}}_{0, T}$. This defines a $T$-point of $\nabla \mathscr{H}^{1}(C, \mathfrak{G})^{\bar{\nu}}$ which we denote $\hat{f}_{1}^{*} \underline{\mathcal{G}}_{0, T}$. Iterating this procedure for $i=2 \ldots n$ we obtain a global $\mathfrak{G}$-shtuka $\hat{f}_{n}^{*} \circ \cdots \circ \hat{f}_{1}^{*} \underline{\mathcal{G}}_{0, T}$ in $\nabla \mathscr{H}^{1}(C, \mathfrak{G})^{\underline{\underline{\nu}}}(T)$. This establishes the desired 1-morphism.

When $S=$ Spec $k$ then the functor $\underline{\mathcal{M}}_{\underline{\mathcal{L}}_{\nu}}$ is represented by $\widehat{\mathcal{F}}_{\mathbb{P}_{\nu}}$, see proposition 4.1.8.

The étaleness of the morphism over its image is just another way of phrasing the rigidity of quasi-isogenies (proposition 3.1.3). Lets explain this more explicitly. Let $\underline{\mathcal{G}}$ be a $T$-valued point of $\nabla_{n} \mathscr{H}^{1}(C, \mathfrak{G})^{\underline{\nu}}$. Let $\bar{T}$ be a closed subscheme of $T$ defined by a locally nilpotent sheaf of ideals. Further assume that $\underline{\mathcal{G}}_{\bar{T}}=\hat{\bar{f}}_{n}^{*} \circ \cdots \circ \hat{\bar{f}}_{1}^{*} \underline{\mathcal{G}}_{0, \bar{T}}$ for the quasi-isogenies $\hat{\bar{f}}_{i}$ (as in the above) defined over $\bar{T}$. Now these quasi-isogenies lift to quasi-isogenies $\hat{f}_{i}$ over $T$ by rigidity of quasi-isogenies. The $T$-valued point $\hat{f}_{n}^{*} \circ \cdots \circ \hat{f}_{1}^{*} \underline{\mathcal{G}}_{0, T}$ of $\nabla_{n} \mathscr{H}^{1}(C, \mathfrak{G})^{\bar{\nu}}$ precisely lifts $\underline{\mathcal{G}}_{\bar{T}}$ to $T$ and thus is identical to $\underline{\mathcal{G}}$. 
It only remains to verify that $\Psi$ is ind-proper. Since $\widehat{\mathcal{F}} \ell_{\mathbb{\nu}_{\nu_{i}}}$ is of ind-finite type, to test the ind-properness of $\Psi$ we can use the valuative criterion of properness, see [L-M, Theorem 7.3].

Let $R$ be a strictly henselian valuation ring with fraction field $L$. Let $\mathcal{G}$ be an $R$ valued point of $\nabla_{n} \mathscr{H}^{1}(C, \operatorname{GL}(\mathcal{V}))^{\overline{\underline{\nu}}}$ and set $\underline{\widehat{\Gamma}}(\underline{\mathcal{G}})=\left(\underline{\mathcal{L}}_{\nu}\right)_{\nu}$. Since $R$ is strictly henselian, for each local $\mathbb{P}_{\nu}$-shtuka $\underline{\mathcal{L}}_{\nu}$ we may take a trivialization $\underline{\mathcal{L}}_{\nu} \cong\left(\left(L^{+} \mathbb{P}\right)_{R}, b_{\nu}^{\prime} \sigma^{*}\right)$ over $R$.

Consider an $L$-valued point $\underline{x}$ of $\prod_{\nu_{i}} \widehat{\mathcal{F}}_{\mathbb{P}_{\nu_{i}}}$ which maps to $\underline{\mathcal{G}}_{L}$ under the morphism $\Psi$ and represent it as tuple $\left(\left(\left(L^{+} \mathbb{P}\right)_{L}, b_{\nu}^{\prime} \sigma^{*}\right), g_{\nu}\right)_{\nu}$, where $g_{\nu}$ lie in $L G(L)$, see proposition 4.1.8.

We may take a faithful representation $\rho: \mathfrak{G} \rightarrow \mathrm{GL}(\mathcal{V})$, where $\mathcal{V}$ is a vector bundle over $C$ of rank $r$, see proposition 4.1.3. Note that this proposition ensures the existence of such representation only locally, but one can patch the local data to obtain the representation globally on $C$ by fppf-descent. Let $\rho_{*} \underline{\mathcal{G}}_{0}$ be the induced global GL( $\left.\mathcal{V}\right)$-shtuka over $S$. Note that we want to show that there is a unique morphism $\tilde{\alpha}$ which fits into the following

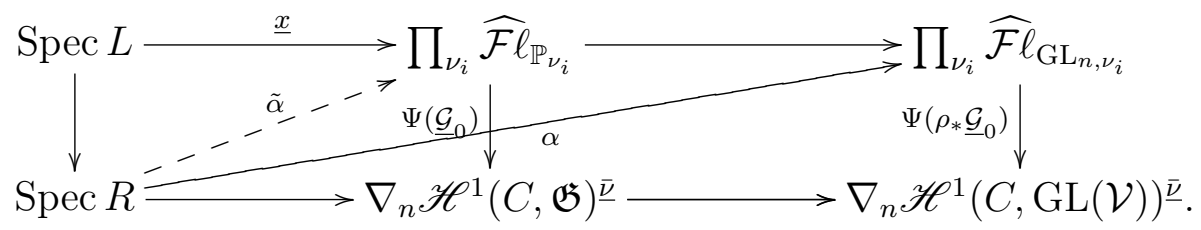

commutative diagram. Here $\operatorname{Spec} L \rightarrow \operatorname{Spec} R$ is the generic point and the horizontal arrows in the right commutative diagram are induced by the representation $\rho$, in addition the existence and uniqueness of the morphism $\alpha$ follows from the fact that $\widehat{\mathcal{F}}_{\mathrm{GL}_{r, \nu_{i}}}$ is ind-proper over $\operatorname{Spf} A_{\nu_{i}}$ and that $\nabla_{n} \mathscr{H}^{1}(C, \mathrm{GL}(\mathcal{V}))^{\overline{\underline{\nu}}}$ is ind-separated, see remark 4.1.4 and proposition 6.2.18. The $R$-valued point $\alpha$ is given by a tuple $\left(\left(\rho_{*}\left(L^{+} \mathbb{P}\right)_{R}, \rho\left(b_{\nu}^{\prime}\right) \sigma^{*}\right), \widetilde{g}_{\nu}\right)_{\nu}$, where $\widetilde{g}_{\nu}$ lies in $L \mathrm{GL}_{n}(R)$, see proposition 4.1.5. Note that the element $\widetilde{g}_{\nu}$ lies in fact in $L G(R)$ (for this notice that $L G$ is closed in $L \mathrm{GL}_{n}$ and $\widetilde{g}_{\nu}$ extends $g_{\nu}$ over $R$ ) and thus produces a lift of $\left(\left(L^{+} \mathbb{P}, b_{\nu}^{\prime} \sigma^{*}\right), g_{\nu}\right)_{\nu}$ over $R$. This gives the desired morphism $\tilde{\alpha}$. Note that the commutativity of the diagram

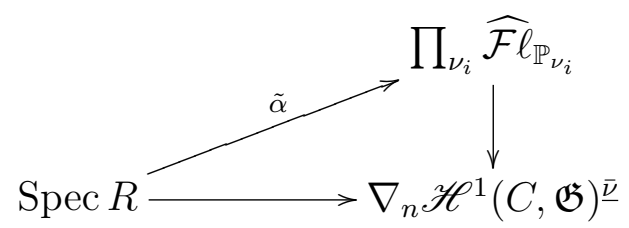


follows from the separatedness of the stack $\nabla_{n} \mathscr{H}^{1}(C, \mathfrak{G})^{\underline{\nu}}$, see proposition 6.2.18.

Remark 4.1.13. One can observe that the image of $\Psi\left(\underline{\mathcal{G}}_{0}\right)$ lies inside the quasi-isogeny locus of $\underline{\mathcal{G}}_{0}$ in $\nabla_{n} \mathscr{H}^{1}(C, \mathfrak{G})^{\underline{\underline{\nu}}}$. Indeed, by the above construction, starting with an $S$-valued point $\underline{x}=\left(\left(\underline{\mathcal{L}}_{\nu_{i}}^{\prime}\right), \hat{f}_{i}\right)_{i}$ of $\prod_{\nu_{i}} \underline{\mathcal{M}}_{\underline{\mathcal{L}}_{\nu_{i}}}(S)$ there is a unique quasi-isogeny $\varrho_{\underline{x}}: \Psi(\underline{x})=\hat{f}_{n}^{*} \circ \cdots \circ \hat{f}_{1}^{*} \underline{\mathcal{G}}_{0} \rightarrow \underline{\mathcal{G}}_{0, S}$ which is an isomorphism outside the graphs of the $\nu_{i}$ with $\left(\underline{\widehat{\Gamma}}(\Psi(\underline{x})), \widehat{\underline{\Gamma}}\left(\varrho_{\underline{x}}\right)\right)=\underline{x}$.

\subsection{The Analogue of the Serre-Tate Theo- rem}

The Serre-Tate Theorem relates the deformation theory of an abelian variety in characteristic $p$ to the deformation theory of the associated $p$-divisible group. In this section we introduce the analogous situation over function fields and prove the analogous theorem relating the deformation theory of a global $\mathfrak{G}$-shtuka to the deformation theory of the associated local $\mathbb{P}_{\nu}$-shtukas via the global-local functor.

Let $S$ be in $\mathcal{N} i l p_{\mathbb{F}_{q} \llbracket \zeta \rrbracket}$ and $i: \bar{S} \rightarrow S$ be a closed subscheme defined by a locally nilpotent sheaf of ideals. Let $\underline{\mathcal{G}}$ be a global $\mathfrak{G}$-shtuka in $\mathfrak{S} h t \frac{\mathcal{\nu}}{\mathfrak{G}}(\bar{S})$. The category $\operatorname{Defo}_{S}(\underline{\overline{\mathcal{G}}})$ of lifts of $\underline{\mathcal{G}}$ to $S$ consists of all pairs $\left(\underline{\mathcal{G}}, \alpha: i^{*} \underline{\mathcal{G}} \rightarrow\right.$ $\overline{\mathcal{G}})$ where $\underline{\mathcal{G}}$ belongs to $\mathfrak{S} h t \underline{\underline{\mathcal{L}}}(S)$ and $\alpha$ is an isomorphism of global $\mathfrak{G}$ shtukas over $\bar{S}$, and morphisms are isomorphisms between the $\underline{\mathcal{G}}$ 's that are compatible with the $\alpha$ 's.

Similarly for a local $\mathbb{P}$-shtuka $\underline{\widehat{\mathcal{G}}}$ in $\widehat{\mathfrak{S} h t} t_{\mathbb{P}}(\bar{S})$ we define the category of lifts $\operatorname{Defo}_{S}(\underline{\widehat{\mathcal{G}}})$ of $\widehat{\widehat{\mathcal{G}}}$ to $S$.

Notice that according to the rigidity of quasi-isogenies all Hom-sets in these categories contain at most one element.

Theorem 4.2.1. Let $\underline{\mathcal{G}}:=(\overline{\mathcal{G}}, \bar{\tau})$ be a global $\mathfrak{G}$-shtuka in $\mathfrak{S} h t_{\mathfrak{G}}(\bar{S})$. Let $\left(\widehat{\mathcal{G}}_{\nu}\right)=\underline{\widehat{\Gamma}}(\underline{\overline{\mathcal{G}}})$. Then the functor

$$
\operatorname{Defo}_{S}(\underline{\overline{\mathcal{G}}}) \rightarrow \prod_{\nu} \operatorname{Defo}_{S}\left(\underline{\overline{\mathcal{G}}}_{\nu}\right)
$$

induced by the global-local functor, is an equivalence of categories. 
Proof. We proceed by constructing the inverse of the above functor. It suffices to treat the case where $\mathcal{I}^{q}=(0)$. In this case the morphism $\hat{\sigma}_{S}$ factors through $i: \bar{S} \rightarrow S$

$$
\hat{\sigma}_{S}=i \circ \sigma^{\prime}: S \rightarrow \bar{S} \rightarrow S
$$

Let $\left(\underline{\widehat{\mathcal{G}}}_{\nu}, \widehat{\alpha}_{\nu}: i^{*} \widehat{\widehat{\mathcal{G}}}_{\nu} \rightarrow \widehat{\widehat{\mathcal{G}}}_{\nu}\right)_{\nu}$ be an object of $\prod_{\nu} \operatorname{Defo} o_{S}\left(\widehat{\overline{\mathcal{G}}}_{\nu}\right)$.

Consider the global $\mathfrak{G}$-shtuka $\underline{\widetilde{\mathcal{G}}}:=\sigma^{*} \underline{\overline{\mathcal{G}}}$ over $S$. The morphisms $\bar{\tau}$ defines an isogeny $\bar{\tau}: i^{*} \widetilde{\mathcal{G}} \rightarrow \overline{\mathcal{G}}$ which is an isomorphism outside the graphs of the characteristic sections

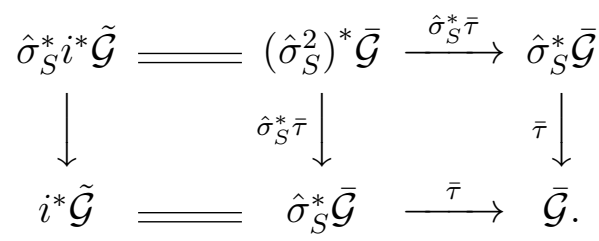

Composing $\hat{\bar{\tau}}_{\nu}^{-1}$ with $\hat{\alpha}_{\nu}$ we obtain $\hat{\bar{\gamma}}_{\nu}:=\hat{\bar{\tau}}_{\nu}^{-1} \circ \hat{\alpha}_{\nu}: i^{*} \widehat{\mathcal{G}}_{\nu} \rightarrow i^{*} \widehat{\widetilde{\mathcal{G}}}_{\nu}$. By rigidity of quasi-isogenies (proposition 3.1.3), the resulting quasi-isogeny of local $\mathbb{P}_{\nu}$-shtukas lifts to a quasi-isogeny $\hat{\gamma}_{\nu}: \underline{\widehat{\mathcal{G}}}_{\nu} \rightarrow \underline{\widetilde{\mathcal{G}}}_{\nu}$.

We put $\underline{\mathcal{G}}:=\Psi(\widetilde{\mathcal{G}})\left(\left(\underline{\mathcal{G}}_{\nu}, \hat{\gamma}_{\nu}\right)\right)$ (see theorem 4.1.12) and recall that there is a quasi-isogeny $\gamma: \underline{\mathcal{G}} \rightarrow \underline{\mathcal{G}}$ of global $\mathfrak{G}$-shtukas, see remark 4.1.13.

We may now define the functor

$$
\prod_{\nu} \operatorname{Defo}\left(\widehat{\overline{\mathcal{G}}}_{\nu}\right) \rightarrow \operatorname{Defo}(\underline{\overline{\mathcal{G}}})
$$

by sending $\left(\left(\underline{\mathcal{G}}_{\nu}, \widehat{\alpha}: i^{*} \widehat{\mathcal{G}}_{\nu} \rightarrow \widehat{\mathcal{\mathcal { G }}}_{\nu}\right)\right)_{\nu}$ to $\left(\underline{\mathcal{G}}, \bar{\tau} \circ i^{*} \gamma\right)$. The quasi-isogeny $\alpha:=$ $\bar{\tau} \circ i^{*} \gamma$ is an isomorphism outside the graphs of the $\nu_{i}$ by remark 4.1.13 and at these graphs by construction. It can be seen by the above construction that these functors are actually inverse to each other. 
38CHAPTER 4. UNBOUNDED UNIFORMIZATION AND THE DEFORMATION THEORY 


\section{Chapter 5}

\section{Galois Representations and Shtukas}

\subsection{The Tate Functor}

Definition 5.1.1. Let $V e c t_{\mathbb{D}}\left(\right.$ resp. $\left.V e c t_{\dot{\mathbb{D}}}\right)$ be the groupoid on $\mathcal{N} i p_{k \llbracket \zeta \rrbracket}$ whose $S$-valued points is the category of locally free sheaves of $\mathcal{O}_{S} \llbracket z \rrbracket-$ modules (resp. $\mathcal{O}_{S}((z))$-modules) of finite rank on $S$. Consider the natural 1-morphism $V e c t_{\mathbb{D}} \rightarrow V e c t_{\mathbb{D}}$ of groupoids. Let $S h t_{\mathbb{D}}$ denote the groupoid

$$
\operatorname{ker}\left(p r_{1}, \hat{\sigma} \circ p r_{2}: V e c t_{\mathbb{D}} \times_{V e c t_{\mathbb{D}}} V e c t_{\mathbb{D}} \rightrightarrows V e c t_{\mathbb{D}}\right)
$$

(see Def 2.2.7)where $p r_{i}$ denotes the projections to the i-th factor.

We represent an object $\left(\sigma^{*} M, M, \varphi\right)$ of the category $S h t_{\mathbb{D}}(S)$ by the pair $(M, \varphi)$.

Definition 5.1.2. A quasi-isogeny $(M, \varphi) \rightarrow\left(M^{\prime}, \varphi^{\prime}\right)$ is an isomorphism of $\mathcal{O}_{S}((z))$-modules

$$
f: M \otimes_{\mathcal{O}_{S} \llbracket z \rrbracket} \mathcal{O}_{S}((z)) \stackrel{\sim}{\longrightarrow} M^{\prime} \otimes_{\mathcal{O}_{S} \llbracket z \rrbracket} \mathcal{O}_{S}((z))
$$

with $\varphi^{\prime} \sigma^{*}(f)=f \varphi$. The category of local shtukas over $S, \widehat{\mathfrak{S h}}_{\mathbb{D}}(S)$, is the category which has $O b\left(S h t_{\mathbb{D}}(S)\right)$ as its objects and quasi-isogenies as its morphisms. Any object in this category is called a local shtuka over $S$.

Remark 5.1.3. Note that there is an equivalence of categories between the category of local $G l_{n}$-shtukas over $S$ and the category of rank $n$ local shtukas over $S$, see section 4 of $[\mathrm{H}-\mathrm{V}]$. 
In analogy with $p$-divisible groups and abelian varieties, one can also assign a Galois representation to a given local or global shtuka. Let us explain this more explicitly.

Assume that $S$ is connected. Let $\bar{s}$ be a geometric point of $S$ and let $\pi_{1}(S, \bar{s})$ denote the algebraic fundamental group of $S$ at $\bar{s}$. We define the Tate functor from the category of local shtukas $\widehat{\mathfrak{S} h t} t_{\mathbb{D}}(S)$ over $S$ to the category of $\mathbb{F}_{q} \llbracket z \rrbracket[\pi(S, \bar{s})]$-modules $\mathfrak{M o d}_{\mathbb{F}_{q} \llbracket z \rrbracket[\pi(S, \bar{s})]}$ as follows

$$
\begin{aligned}
T_{-}: \operatorname{Sht}_{\mathbb{D}}(S) & \rightarrow \operatorname{Mod}_{\mathbb{F}} \llbracket z \rrbracket[\pi(S, \bar{s})] \\
\underline{M}:=(M, \tau) & \mapsto \quad T_{\underline{M}}:=(M \otimes \kappa(\bar{s}))^{\tau} .
\end{aligned}
$$

Here the superscript $\tau$ represents the $\tau$-invariants.

Remark 5.1.4. Like in $p$-adic Hodge theory, the restriction of the above functor to the category of étale local shtukas is in fact an equivalence of categories, see [Kat, Prop 4.1.1] also [Ha2, Prop 1.3.7]. This can be thought as a positive characteristic analogue of the Riemann-Hilbert correspondence.

Let $V$ be a free $k \llbracket z \rrbracket$-module of finite rank. A representation $\rho: \mathbb{P} \rightarrow$ $G l(V)$ gives a functor

$$
\rho_{*}: \mathscr{H}^{1}\left(\operatorname{Spec} k, L^{+} \mathbb{P}\right) \rightarrow V e c t_{\mathbb{D}}
$$

which sends an $L^{+} \mathbb{P}$-torsor $\mathcal{L}_{+}$to the sheaf associated with the following presheaf

$$
Y \longmapsto\left(\mathcal{L}_{+}(Y) \times\left(V \otimes_{k} \mathcal{O}_{S} \llbracket z \rrbracket(Y)\right)\right) / L^{+} \mathbb{P}(Y)
$$

The functor $\rho_{*}: \mathscr{H}^{1}\left(\operatorname{Spec} k, L^{+} \mathbb{P}\right) \rightarrow V$ ect $t_{\mathbb{D}}$ induces a morphism from the category of local $\mathbb{P}$-shtukas $\widehat{\mathfrak{S} h} t_{\mathbb{P}}$ to the category $\widehat{\mathfrak{S} h}_{\mathbb{D}}$ of local shtukas. We still use the same notation $\rho_{*}: \widehat{\mathfrak{S} h}_{\mathbb{P}} \rightarrow \widehat{\mathfrak{S} h t_{\mathbb{D}}}$.

Let Funct $^{\otimes}\left(\operatorname{Rep}_{k \llbracket z \rrbracket} \mathbb{P}, \mathfrak{M o d}_{\mathbb{F}_{q} \llbracket z \rrbracket[\pi(S, \bar{s})]}\right)$ denote the category of tensor functors from the category of representations of $\mathbb{P}$ in finite free $k \llbracket z \rrbracket$-modules, $\operatorname{Rep}_{k \llbracket z \rrbracket} \mathbb{P}$, to $\mathfrak{M o d}_{\mathbb{F}_{q} \llbracket z \rrbracket[\pi(S, \bar{s})]}$.

We proceed by defining, the Tate functor as the following tensor functor

$$
\begin{aligned}
\widehat{\mathcal{T}}_{-}: \widehat{\mathfrak{S h}}_{\mathbb{P}}(S) & \rightarrow \text { Funct }^{\otimes}\left(\operatorname{Rep}_{k \llbracket z \rrbracket} \mathbb{P}, \mathfrak{M o d}_{\mathbb{F}_{q} \llbracket z \rrbracket[\pi(S, \bar{s})]}\right) \\
\underline{\mathcal{L}} & \mapsto \mathcal{T}_{\mathcal{L}}: \rho \mapsto T_{\rho_{*} \underline{\mathcal{L}}}
\end{aligned}
$$




\subsection{The Level Structure}

Fix a tuple $\underline{\nu}=\left(\nu_{1}, \ldots, \nu_{n}\right)$ of places on $C$. Let the affine curve $C^{\prime}$ be the complement in $C$ of the characteristic places $\nu_{i}$. Let $\underline{\mathcal{G}}$ be a global $\mathfrak{G}$-shtuka in $\mathfrak{S} h t \underline{\underline{L}}(S)$. For a finite subscheme $D$ of $C$ let $\left.\underline{\mathcal{G}}\right|_{D}$ denote the pullback of $\underline{\mathcal{G}}$ to $D \times S$.

Let Funct $^{\otimes}\left(\operatorname{Rep}_{\mathbb{A} \nu} \mathfrak{G}, \mathfrak{M}_{o} d_{\mathbb{A} \nu[\pi(S, s)]}\right)$ denote the category of tensor functors from the category $\operatorname{Rep}_{\mathbb{A} \underline{\underline{\nu}}} \mathfrak{G}$ of finite dimentional representations of $\mathfrak{G}$ over the adelic ring $\mathbb{A} \underline{\nu}$ of $C$, to the category of $\mathbb{A} \underline{\nu}[\pi(S, \bar{s})]$-modules $\mathfrak{M} d_{\mathbb{A}} \underline{\underline{L}}[\pi(S, \bar{s})]$. We define the Tate functor as follows

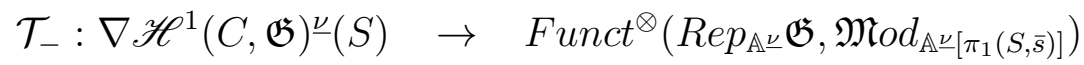

$$
\begin{aligned}
& \underline{\mathcal{G}} \mapsto \mathcal{T}_{\underline{\mathcal{G}}}: \rho:=\left(\rho_{\nu}\right) \mapsto \lim _{\overleftarrow{D \subset C^{\prime}}}\left(\left.\rho_{*} \underline{\mathcal{G}}\right|_{D \times \bar{s}}\right)^{\tau} .
\end{aligned}
$$

Remark 5.2.1. Note that there is an isomorphism

$$
\lim _{\overline{D \subset C^{\prime}}}\left(\left.\rho_{*} \underline{\mathcal{G}}\right|_{D \times \bar{s}}\right)^{\tau} \simeq \prod_{\nu \in C^{\prime}} T_{\rho_{\nu *} \hat{\underline{\underline{q}}}_{\nu}}
$$

see remark 3.2.4.

Recall that as a consequence of Tannakian theory, there is a duality between affine group schemes over arbitrary field and the neutral tannakian categories. More precisely one may recover such groups from the associated category of representations. In [Wed] T. Wedhorn establishes a generalization of the theory to the case where the base is a Prüfer ring (e.g. a Dedekind ring).

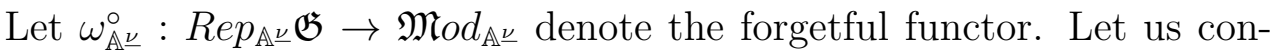
sider the set of isomorphisms of tensor functors $\operatorname{Isom}^{\otimes}\left(\mathcal{T}_{\underline{\mathcal{G}}}, \omega_{\AA ⿻}^{\circ}\right.$ ) (note that this set is non-empty, see lemma 5.2 .2 below). By the generalized tannakian formalism and the definition of the Tate functor, this set admits a biaction of $\mathfrak{G}(\mathbb{A} \underline{\nu}) \times \pi_{1}(S, \bar{s})$.

Lemma 5.2.2. Let $\hat{\mathbb{P}}$ be as in the definition 3.2.1. Then for any $b \in \hat{\mathbb{P}}(\bar{k} \llbracket z \rrbracket)$ there exist some $c \in \hat{\mathbb{P}}(\bar{k} \llbracket z \rrbracket)$ such that $b \sigma^{*} c=c$.

Proof. Let us view $\hat{\mathbb{P}}$ as the direct limit $\underset{n}{\lim } \mathbb{P}_{n}$, where $\mathbb{P}_{n}=\mathbb{P} \otimes_{\mathbb{D}} \mathbb{D}_{n}$. Let $\widetilde{G}_{n}$ denote the linear algebraic group over $\bar{k}$ given by the Weil restriction 
$\operatorname{Res}_{\mathbb{D}_{n} / \operatorname{Spec} k}\left(\mathbb{P}_{n}\right)$. The reduction of $b \bmod z^{n}$ gives an element $b_{n} \in \widetilde{G}_{n}(\bar{k})$. By Lang's theorem [Lan] there exist $c_{n} \in \widetilde{G}_{n}(\bar{k})$ such that $b_{n} \hat{\sigma}^{*} c_{n}=c_{n}$, where $\hat{\sigma} \in \operatorname{Gal}(\bar{k} / k)$ is the Frobenius element. Now consider the reduction map $\alpha_{n}: \widetilde{G}_{n+1}(\bar{k}) \rightarrow \widetilde{G}_{n}(\bar{k})$. Consider the element $\bar{d}_{n}:=\alpha_{n}\left(c_{n+1}\right)^{-1} c_{n}$ which satisfies $\hat{\sigma}^{*} \bar{d}_{n}=\bar{d}_{n}$ and hence lies in $\widetilde{G}_{n}(k)=\mathbb{P}\left(\mathbb{D}_{n+1}\right)$. Since $\mathbb{P}$ is smooth $\bar{d}_{n}$ lifts to an element $d_{n} \in \mathbb{P}\left(\mathbb{D}_{n+1}\right)=\widetilde{G}_{n+1}(k)$. Replacing $c_{n+1}$ by $c_{n+1} d_{n}$ we may assume that $\alpha_{n}\left(c_{n+1}\right)=c_{n}$ and then take $c:=\underset{\leftarrow}{\lim } c_{n}$.

Definition 5.2.3. Let $H \subseteq \mathfrak{G}\left(\mathbb{A}^{\underline{\nu}}\right)$ be a compact open subgoup. An $H$-level structure on a global $\mathfrak{G}$-shtuka $\underline{\mathcal{G}}$ over $S$ is a $\pi_{1}(S, \bar{s})$-invariant $H$-orbit in $\operatorname{Isom}^{\otimes}\left(\mathcal{T}_{\underline{\mathcal{G}}}, \omega_{\AA}^{\circ \underline{\nu}}\right)$.

Now we want to establish the notion of rational level structure $H \subseteq$ $\mathfrak{G}\left(\mathbb{A}_{Q}\right)$ on a global $\mathfrak{G}$-shtuka $\mathcal{G}$ in $\mathfrak{S} h t_{\mathfrak{G}}^{\nu}(S)$.

A given quasi-isogeny $\varrho: \underline{\mathcal{G}} \rightarrow \underline{\mathcal{G}}^{\prime}$ in $\mathfrak{S} h t_{\mathfrak{G}}(S)$ induces a morphism

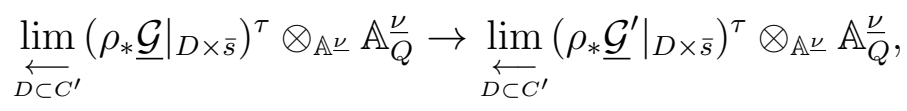

thus the Tate functor we defined above extends to the following functor

$$
\begin{aligned}
& \mathcal{V}_{-}: \mathfrak{S} h t_{\mathfrak{G}}^{\nu}(S) \rightarrow \operatorname{Funct}^{\otimes}\left(\operatorname{Rep}_{\mathbb{A}} \frac{\nu}{Q} \mathfrak{G}, \mathfrak{M o d} d_{\mathbb{A}} \frac{\nu}{Q}\left[\pi_{1}(S, \bar{s})\right]\right) \\
& \underline{\mathcal{G}} \mapsto \quad \mathcal{V}_{\underline{\mathcal{G}}}: \rho:=\left(\rho_{\nu}\right) \mapsto \lim _{\overleftarrow{D \subset C^{\prime}}}\left(\left.\rho_{*} \underline{\mathcal{G}}\right|_{D \times \bar{s}}\right)^{\tau} \otimes_{\mathbb{A} \underline{\nu}} \mathbb{A} \frac{\nu}{Q} .
\end{aligned}
$$

Note that $\lim _{\overline{D \subset C^{\prime}}}\left(\left.\rho_{*} \underline{\mathcal{G}}\right|_{D \times \bar{s}}\right)^{\tau} \otimes_{\mathbb{A} \underline{\nu}} \mathbb{A} \frac{\nu}{Q} \simeq \prod_{\nu \in C^{\prime}} T_{\rho_{\nu *} \underline{\mathcal{G}}_{\nu}} \otimes_{\mathbb{A} \underline{\nu}} \mathbb{A} \frac{\nu}{Q}$

Let $\omega^{\circ}: \operatorname{Rep}_{\mathbb{A}} \frac{\nu}{Q} \mathfrak{G} \rightarrow \mathfrak{M o d}_{\mathbb{A}} \frac{\nu}{Q}$ denote the forgetful functor. Let us consider the set of isomorphisms of tensor functors $\operatorname{Isom}^{\otimes}\left(\mathcal{V}_{\underline{\mathcal{G}}}, \omega^{\circ}\right)$. Again by the tannakian formalism and the definition of the Tate functor this set admits a biaction of $\mathfrak{G}\left(\mathbb{A} \frac{\nu}{Q}\right) \times \pi_{1}(S, \bar{s})$.

Now for a compact open subgroup $H \subseteq \mathfrak{G}\left(\mathbb{A} \frac{\nu}{Q}\right)$ one can define an $H$ level structure on a global $\mathfrak{G}$-shtuka $\underline{\mathcal{G}}$ over $S$ as a $\pi_{1}(S, \bar{s})$-invariant $H$ orbit in $\operatorname{Isom}^{\otimes}\left(\mathcal{V}_{\mathcal{G}}, \omega^{\circ}\right)$. We denote by $\nabla_{n}^{H} \mathscr{H}^{1}(C, \mathfrak{G})$ the category fibered in groupoids who's $\bar{S}$-valued points are tuples $(\mathcal{G}, \gamma)$, consisting of a $\mathfrak{G}$-shtuka $\underline{\mathcal{G}} \in O b\left(\mathfrak{S} h t_{\mathfrak{G}}^{\underline{\nu}}\right)$ together with a rational level $H$-structure $\gamma$ as its objects, and has quasi-isogenies that are isomorphisms at characteristic places $\nu_{i}$ and are compatible with the level $H$-structures as its morphisms. 
Remark 5.2.4. Let $D$ denote a finite subscheme of $C$. Then one can define a $D$-level structure on the objects of $\operatorname{Hecke}_{\mathfrak{G}, n}(S)$. Namely, for an object $\underline{\mathcal{G}}:=\left(\mathcal{G}, \mathcal{G}^{\prime}, \varphi\right) \in O b\left(\operatorname{Hecke}_{\mathfrak{G}, n}(S)\right)$ we require that the $\mathfrak{G}$-bundles $\mathcal{G}$ and $\mathcal{G}^{\prime}$ lie in $\mathscr{H}_{D}^{1}(C, G)(S)$ and in addition $\varphi$ preserves the $D$-level structures on them. This induces a $D$-level structure on the objects of the moduli stack of global $\mathfrak{G}$-shtukas, we denote the corresponding stack by $\nabla_{n} \mathscr{H}_{D}^{1}(C, \mathfrak{G})^{\underline{\nu}}$. Note that a $D$-level structure on $\underline{\mathcal{G}}$ can give rise to a level structure of the above form which we denote by $\overline{H_{D}}$.

Theorem 5.2.5. There is an isomorphism

$$
\nabla_{n} \mathscr{H}_{D}^{1}(C, \mathfrak{G})^{\underline{\nu}} \stackrel{\sim}{\rightarrow} \nabla_{n}^{H_{D}} \mathscr{H}^{1}(C, \mathfrak{G})^{\underline{\nu}}
$$

of formal stacks.

Proof. Let $(\underline{G}, \psi)$ be an object in $\nabla_{n} \mathscr{H}_{D}^{1}(C, \mathfrak{G})^{\underline{\nu}}(S)$. For any representation $\rho$ in $\operatorname{Rep}_{\mathbb{A}^{\frac{\nu}{Q}}} \mathfrak{G}$ the isomorphism $\psi:\left.\underline{\mathcal{G}}\right|_{D \times S} \rightarrow \mathfrak{G} \times_{C} D \times S$ induces an isomorphism $\left.\rho_{*} \underline{\mathcal{G}}\right|_{D_{S}} \tilde{\rightarrow} G l_{\operatorname{dim} \rho}\left(\mathcal{O}_{D_{S}}\right)$ and consequently we obtain a transformation $\bar{\gamma}: \mathcal{T}_{\underline{\mathcal{G}}} \otimes_{\mathbb{A}^{\underline{\nu}}} \mathcal{O}_{D} \rightarrow \omega^{0} \otimes_{\mathbb{A}^{\underline{\nu}}} \mathcal{O}_{D}$. Let $\gamma$ be a lift of $\bar{\gamma}$ to $\mathbb{A}^{\underline{\nu}}$. Then we define the morphism

$$
\nabla_{n} \mathscr{H}_{D}^{1}(C, \mathfrak{G})^{\underline{\nu}}(S) \stackrel{\sim}{\rightarrow} \nabla_{n}^{H_{D}} \mathscr{H}^{1}(C, \mathfrak{G})^{\underline{\nu}}(S)
$$

by sending $(\underline{\mathcal{G}}, \psi)$ to $\left(\underline{\mathcal{G}}, H_{D} \gamma_{Q}\right)$.

Let us show that this functor is essentially surjective. Let $\left(\underline{\mathcal{G}}, \psi, H_{D} \gamma_{Q}\right)$ be an object of the category $\nabla_{n}^{H_{D}} \mathscr{H}^{1}(C, \mathfrak{G})^{\underline{\nu}}$. Choose $\beta: \mathcal{T}_{\underline{\mathcal{G}}} \stackrel{\sim}{\rightarrow} \omega_{\mathbb{A} \underline{\nu}}^{\circ}$. Note that this exist by lemma 5.2.2.

The automorphism $\gamma_{Q} \beta_{Q}^{-1} \in A u t^{\otimes}\left(\omega_{Q}^{\circ}\right)$ corresponds to an element $g \in$ $\mathfrak{G}\left(\mathbb{A} \frac{\underline{\nu}}{Q}\right)$. Write $g:=\left(g_{x_{1}}, \ldots, g_{x_{r}}, g^{\underline{x}}\right) \in \mathfrak{G}\left(Q_{x_{1}}\right) \times \cdots \times \mathfrak{G}\left(Q_{x_{r}}\right) \times \mathfrak{G}\left(\mathbb{A}^{\underline{\nu}}, \underline{\underline{x}}\right)$ and set $g^{\prime}=g\left(1, \ldots, 1, g^{\underline{x}}\right)^{-1}=\left(g_{x_{1}}, \ldots, g_{x_{r}}, 1\right)$ and $\beta^{\prime}=\left(1, \ldots, 1, g^{\underline{x}}\right) \cdot \beta \in$ $\mathfrak{G}(\mathbb{A} \underline{\nu})$.

Now since the local $\mathbb{P}_{x_{i}}$-shtuka $\underline{\mathcal{G}}_{x_{i}}$ is étale, the automorphism $\delta_{i}:=$ $\left(\beta_{Q}^{\prime}\right)^{-1} g_{x_{i}}^{-1} \beta_{Q}^{\prime} \in A u t^{\otimes}\left(\mathcal{V}_{\underline{\mathcal{G}}}\right)$ gives a quasi-isogeny $\delta_{i}: \underline{\widehat{\mathcal{G}}}_{x_{1}} \rightarrow \underline{\widehat{\mathcal{G}}}_{x_{1}}$. Let us explain this more precisely. Consider the following functor

$$
\widehat{\mathcal{M}}_{-}: \mathscr{H}^{1}\left(\operatorname{Spec} k, L^{+} \mathbb{P}\right)(S) \rightarrow \operatorname{Funct}^{\otimes}\left(\operatorname{Rep}_{k \llbracket z \rrbracket} \mathbb{P}, \mathfrak{M} d_{\mathcal{O}_{S} \llbracket z \rrbracket}\right)
$$

which sends $\mathcal{L}_{+}$to the functor which sends the representation $\rho$ to the $\mathcal{O}_{S} \llbracket z \rrbracket$-module $\rho_{*} \mathcal{L}_{+}$.

Assume that $\underline{\widehat{\mathcal{G}}}=\left(\mathcal{L}_{+}, \hat{\varphi}\right)$ is an étale local $\mathbb{P}$-shtuka. An automorphism $\alpha$ of 
$\mathcal{V}_{\widehat{\mathcal{G}}}$ induces an automorphism of $\widehat{\mathcal{M}}_{\widehat{\mathcal{G}}} \otimes_{\mathcal{O}_{S} \llbracket z \rrbracket} \mathcal{O}_{S} \llbracket z \rrbracket[1 / z]$. Take a trivializing étale cover $S^{\prime} \rightarrow S$. Then we have

$$
A u t^{\otimes}\left(\widehat{\mathcal{M}}_{\mathcal{L}_{+S^{\prime}}}\right)=A u t^{\otimes}\left(\omega^{\circ}\right)\left(\mathcal{O}_{S^{\prime} \llbracket z \rrbracket}[1 / z]\right)=L P_{\eta}\left(S^{\prime}\right) .
$$

Hence the automorphism $\alpha$ gives an isomorphism $h_{S^{\prime}}: L P_{\eta, S^{\prime}} \rightarrow L P_{\eta, S^{\prime}}$. The morphism $h_{S^{\prime}}$ inherits the descent data coming from the fact that $\alpha$ is defined over $S$, and hence it defines an isomorphism $h: \mathcal{L} \rightarrow \mathcal{L}$, where $\mathcal{L}$ denotes the associated $L P_{\eta}$-torsor. One can check that $h$ satisfies $\hat{\sigma}^{*} h=\hat{\varphi}^{-1} \circ h \circ \hat{\varphi}$ and gives the claimed quasi-isogeny.

Let $\underline{\mathcal{G}}^{\prime}=\delta^{*} \underline{\mathcal{G}}$, where $\delta:=\delta_{r} \circ \cdots \circ \delta_{1}$, then

$$
\mathcal{V}(\delta)=\left(\beta_{Q}^{\prime}\right)^{-1}\left(g^{\prime}\right)^{-1} \beta_{Q}^{\prime} .
$$

Consider the pair $\left(\underline{\mathcal{G}}^{\prime}, H_{D} \beta_{Q}^{\prime}\right)$ consisting of the global $\mathfrak{G}$-shtuka $\underline{\mathcal{G}}^{\prime}=$ $\left(\mathcal{G}^{\prime}, \tau^{\prime}\right)$ together with the level structure $\beta^{\prime}: \mathcal{T}_{\mathcal{G}^{\prime}} \rightarrow \omega^{\circ}$ defined over $\mathbb{A} \underline{\nu}$. Note that this is quasi-isogenous to $\left(\underline{\mathcal{G}}, H_{D} \gamma_{Q}\right)$ under $\delta$.

We now want to show that $\left(\underline{\mathcal{G}}^{\prime}, H_{D} \hat{\beta}_{Q}^{\prime}\right)=\left(\left(\mathcal{G}^{\prime}, \varphi^{\prime}\right), H_{D} \beta_{Q}^{\prime}\right)$ actually comes from a pair $\left(\underline{\mathcal{G}}^{\prime}, \psi_{D}\right)$ in $\nabla_{n} \mathscr{H}_{D}^{1}(C, \mathfrak{G})^{\underline{\nu}}$.

Consider the following functor

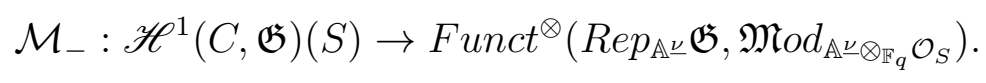

The $H_{D}$ orbit of $\beta^{\prime}$ in $\operatorname{Funct}^{\otimes}\left(\operatorname{Rep}_{\mathbb{A} \underline{\underline{\nu}}} \mathfrak{G}, \mathfrak{M} \mathrm{dod}_{\mathbb{A} \underline{\underline{\nu}}\left[\pi_{1}(S, \bar{s})\right]}\right)$ induces a well defined isomorphism

$$
\left(\mathcal{M}_{\mathcal{G}^{\prime}} \otimes_{\mathcal{O}_{C_{S}}} \mathcal{O}_{D \times \bar{s}}\right)^{\tau^{\prime}} \rightarrow \omega^{\circ} \otimes_{\mathbb{A} \underline{\underline{\nu}}} \mathcal{O}_{D_{S}}
$$

Tensoring up with $\mathcal{O}_{S}$ and observing $\left(\mathcal{M}_{\mathcal{G}^{\prime}} \otimes_{\mathcal{O}_{C_{S}}} \mathcal{O}_{D \times \bar{s}}\right)^{\tau^{\prime}} \otimes_{\mathcal{O}_{D}} \mathcal{O}_{D_{S}} \cong \mathcal{M}_{\mathcal{G}_{D_{S}}^{\prime}}$ we get an isomorphism $\mathcal{M}_{\mathcal{G}_{D_{S}}^{\prime}} \stackrel{\sim}{\rightarrow} \omega^{\circ} \otimes_{\mathbb{A} \underline{\nu}} \mathcal{O}_{D_{S}}$ of tensor functors. Now we claim that this trivialization induces a trivialization $\psi_{D}: \mathcal{G}_{D_{S}}^{\prime} \stackrel{\sim}{\rightarrow} \mathfrak{G} \times_{C} D_{S}$ of the torsor $\mathcal{G}_{D_{S}}^{\prime}$. Note that since the trivialization of the functor $\mathcal{M}_{\mathcal{G}_{D_{S}}^{\prime}}$ is compatible with the Frobenius automorphism $\bar{\tau}^{\prime}$, i.e. the following diagram

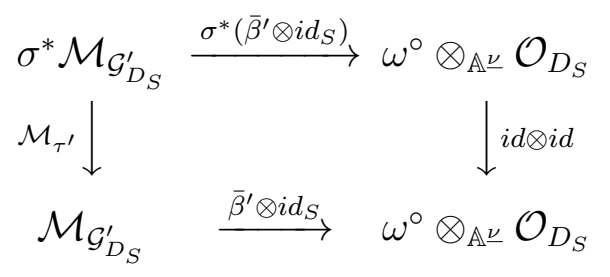

commutes, we may argue that the trivialization $\mathcal{M}_{\mathcal{G}_{D_{S}}^{\prime}} \stackrel{\sim}{\rightarrow} \omega^{\circ} \otimes_{\mathbb{A} \underline{\nu}} \mathcal{O}_{D_{S}}$ of the functor $\mathcal{M}_{\mathcal{G}_{D_{S}}^{\prime}}$ even induces a level structure $\psi_{D}:\left(\underline{\mathcal{G}}_{D_{S}}^{\prime}, \bar{\tau}^{\prime}\right) \stackrel{\sim}{\rightarrow}\left(\mathfrak{G} \otimes_{C} D_{S}, i d\right)$. 
So it remains to verify the above claim. To see this take a trivialization $\varphi^{\prime}: \mathcal{G}_{D_{S^{\prime}}}^{\prime} \tilde{\rightarrow} \mathfrak{G} \times_{C} D_{S^{\prime}}$ over an étale covering $S^{\prime} \rightarrow S$. By the Tannakian formalism we obtain an element $\psi^{\prime} \in \mathfrak{G}\left(D_{S^{\prime}}\right)$ corresponding to the following automorphism

$$
\omega^{\circ} \otimes \mathcal{O}_{D_{S^{\prime}}}=\mathcal{M}_{\mathfrak{G} \times_{C} D_{S^{\prime}}} \stackrel{\varphi^{\prime-1}}{\longrightarrow} \mathcal{M}_{\mathcal{G}_{D_{S^{\prime}}}^{\prime}} \stackrel{\bar{\beta}^{\prime}}{\rightarrow} \omega^{\circ} \otimes \mathcal{O}_{D_{S^{\prime}}} .
$$

of the tensor functor $\omega^{0} \otimes_{\mathbb{A}^{\underline{\nu}}} \mathcal{O}_{D_{S^{\prime}}}$. Then since $\mathcal{M}_{\mathcal{G}_{D_{S}}^{\prime}} \sim \omega^{\circ} \otimes_{\mathbb{A}^{\underline{\nu}}} \mathcal{O}_{D_{S}}$ is defined over $S$, the morphism $\psi^{\prime}: \mathfrak{G} \times_{C} D_{S^{\prime}} \rightarrow \mathfrak{G} \times_{C} D_{S^{\prime}}$ carries the descent data and therefore it descends to an isomorphism $\psi_{D}: \mathcal{G}_{D_{S}}^{\prime} \rightarrow \mathfrak{G} \times_{C} D_{S}$. This

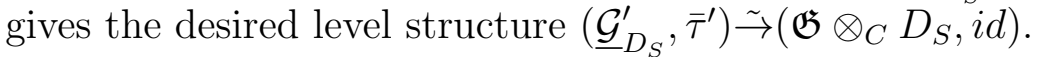

Analyzing this construction further also shows that the functor (5.2.1) is fully faithful. This proves the theorem.

Proposition 5.2.6. Let $\mathfrak{G}$ be a parahoric Bruhat-Tits group scheme over C. Let $\widetilde{H}, H \subseteq \mathfrak{G}\left(\mathbb{A}^{\underline{\nu}}\right)$ be compact open subgroups and assume that $\widetilde{H}$ is a normal subgroup of $H$. Then the stack $\nabla_{n}^{\widetilde{H}} \mathscr{H}^{1}(C, \mathfrak{G})$ is an $H / \widetilde{H}$-torsor over $\nabla_{n}^{H} \mathscr{H}^{1}(C, \mathfrak{G})$ under the projection map.

Proof. One can easily check that the morphism

$$
\begin{aligned}
\nabla_{n}^{\widetilde{H}} \mathscr{H}^{1}(C, \mathfrak{G}) \times H / \widetilde{H} & \rightarrow \nabla_{n}^{\widetilde{H}} \mathscr{H}^{1}(C, \mathfrak{G}) \times \nabla_{n}^{H} \mathscr{H}^{1}(C, \mathfrak{G}) \\
(\underline{\mathcal{G}}, \widetilde{H} \delta) \times g \widetilde{H} & \mapsto \underline{\mathcal{H}} \mathscr{H}^{1}(C, \mathfrak{G}) \\
& \mapsto \underline{H} \delta) \times\left(\underline{\mathcal{G}}, \widetilde{H} g^{-1} \delta\right)
\end{aligned}
$$

is an isomorphism of algebraic stacks. The inverse morphism is given by sending $(\underline{\mathcal{G}}, \widetilde{H} \delta) \times(\underline{\mathcal{G}}, \widetilde{H} \varepsilon)$ to $(\underline{\mathcal{G}}, \widetilde{H} \delta) \times \delta \varepsilon^{-1} \widetilde{H}$. 


\section{Chapter 6}

\section{The Rapoport-Zink Space for Local $\mathbb{P}$-Shtukas}

Rapoport and Zink construct a moduli space for $p$-divisible groups together with a quasi-isogeny to a fixed one (together with some extra structure such as a polarization, endomorphisms, or a level structure). They investigate that this moduli space is pro-representable by a formal scheme locally formally of finite type over $\mathbb{Z}_{p}$.

Previously, in the analogy between mixed and equicharacteristics, we mentioned a sort of resemblance between $p$-divisible groups and local $\mathbb{P}_{-}$ shtukas. It turns out that this analogy is not perfect, unless we restrict to the "bounded" local $\mathbb{P}$-shtukas as the analogous objects corresponding to $p$ divisible groups, for example see 6.3.4. The boundedness condition controls the relative position of two loop torsors (resp. $\mathfrak{G}$-bundles).

Taking bounded local $\mathbb{P}$-shtukas into account, as the right analogs of p-divisible groups, one may naturally seek that the analogous phenomenon, as what we mentioned above for the Rapoport-Zink space for p-divisible groups, also occurs for them. When $\mathbb{P}$ is constant, i.e. $\mathbb{P}:=G_{0} \times \mathbb{D}$ where $G_{0}$ is a reductive group over $\mathbb{F}_{q}$, it turns out to be the case. This has been proven by Hartl and Viehmann, see $[\mathrm{H}-\mathrm{V}]$. Following their approach (after giving an axiomatic definition of boundedness condition), we generalize their result to the non-constant case, i.e. for a parahoric (Bruhat-Tits) group $\mathbb{P}$ over $\mathbb{D}$.

Let us briefly go through the content of this chapter. We first recall some functorial properties of Bruhat-Tits buildings. Then we state a result of Rapoport and Zink (see 6.1.3) which in some sense illustrates the uniform distribution of rational points on the moduli of local $\mathbb{P}$-shtukas. Then we 
discuss the notion of bounds on the quasi-isogenies for both local $\mathbb{P}$-shtukas and global $\mathfrak{G}$-shtukas.

Finally we prove that the Rapoport-Zink space for bounded local $\mathbb{P}$-shtukas is representable by a formal scheme locally formally of finite type, see theorem 6.3.1.

\subsection{Some discussion about Bruhat-Tits build- ing}

Let $G$ be a reductive group over $F$, for a complete discretely valued field $F$ with perfect residue field. Let $\mathscr{B}(G)$ denote the Bruhat-Tits building associated to $G$, recall that this is a complete metric space with respect to the metric $d_{\mathscr{B}}$. The Bruhat-Tits buildings enjoy certain functorial properties

- Functoriality with respect to field extensions, the following easy facts follow from the construction of the Bruhat-Tits building

1) For unramified extension $L / F$ we have a natural metric embedding $\mathscr{B}\left(G_{F}\right) \hookrightarrow \mathscr{B}\left(G_{L}\right)$ of associated Bruhat-Tits buildings, see [BT1, 9.1.19].

2) $L / F$ be a finite Galois extension, then $G(L) \times G a l(L / F)$ acts on $\mathscr{B}\left(G_{L}\right)$, see [BT1, 9.1.19].

3) By the Bruhat-Tits fixed point theorem, one may realise $\mathscr{B}(G, F)$ as the set of fixed points of $\mathscr{B}(G, L)$ under the relative Frobenius automorphism $\tilde{\sigma} \in \operatorname{Gal}(L / F)$.

- Functoriality of buildings with respect to group homomorphisms, this has been worked out by Landvogt, see [La1]. Landvogt proved that a given injective morphism $G \rightarrow G^{\prime}$ of connected reductive groups over $K$ gives rise to a set of $G(k)$-equivariant and isometric maps $f^{*}: \mathscr{B}(G) \rightarrow \mathscr{B}\left(G^{\prime}\right)$. These maps send the apartment associated to a maximal split torus $S$ of $G$ to the apartment associated to a maximal split torus of $G^{\prime}$ containing the image of $S$. This may, in particular, apply to a faithful representation $\rho: G \rightarrow G l_{n}$. This enables us to view $\mathscr{B}(G)$ as a metric subspace of $\mathscr{B}\left(G l_{n}\right)$. For the details and proofs we refer the reader to [La2]. 
Set $F:=k((z))$ and $L:=\bar{k}((z))$. Let $\tilde{\sigma} \in \operatorname{Gal}(L / F)$ be the relative Frobenius automorphism. Let $G$ be a connected reductive algebraic group over $F$ (later we assume that $G$ is the generic fiber $P_{\eta}$ of the parahoric group scheme $\mathbb{P}$ over $\mathbb{D})$.

To an element $b \in G(L)$ Kottwitz associates a slope homomorphism

$$
\nu_{b}: D_{L} \rightarrow G_{L}
$$

called Newton polygon of $b$, see $[K o 1,4.2]$. Here $D$ is the diagonalizable proalgebraic group over $L$ with character group $\mathbb{Q}$. The slope homomorphism is characterized by assigning the slope filtration of $\left(V \otimes_{F} L, \rho(b) .(i d \otimes \tilde{\sigma})\right)$ to any $F$-rational representation $(V, \rho)$ of $G$, see [Ko1, Section 3]. We assume that $b \in G(L)$ satisfies a decency equation, i.e. the following identity

$$
(b \tilde{\sigma})^{s}=s \nu_{b}(z) \cdot \tilde{\sigma}^{s},
$$

in $G(L) \ltimes\langle\tilde{\sigma}\rangle$ for some sufficiently large integer $s>0$.

Remark 6.1.1. Note that any $\tilde{\sigma}$-conjugacy class in G(L) contains an element satisfying a decency equation, see [Ko1, Section 4].

Remark 6.1.2. To the element $b \in G(L)$ one can associate a connected algebraic group $J_{b}$ which is defined by its functor of points that assigns to an $F$-algebra $R$

$$
J_{b}(R):=\left\{g \in G\left(R \otimes_{F} L\right) ; g^{-1} b \tilde{\sigma}(g)=b\right\} .
$$

Let $F_{s}$ be the fixed field of $\tilde{\sigma}^{s}$ in $L$. Then $\nu_{b}$ is defined over $F_{s}$ and $J_{F_{s}}$ is the centralizer of the 1-parameter subgroup $s \nu_{b}$ of $G$ and hence a Levi subgroup of $G_{F_{s}}$, see [RZ, Corollary 1.9]. Thus there is a canonical $J_{b}(L) \rtimes$ $\left\langle\tilde{\sigma}^{s}\right\rangle$-equivariant isometric embedding $\mathscr{B}(J, L) \rightarrow \mathscr{B}(G, L)$ by functoriality of buildings with respect to group homomorphisms.

By the theorem of Bruhat and Tits we have

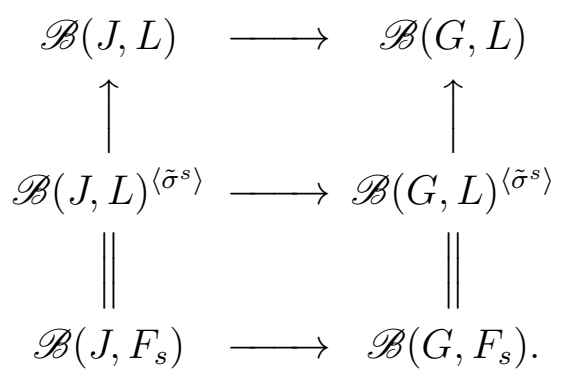


For a given integer $r$ we let $B_{r}(x)$ denote the open ball of radius $r$ centred at $x \in \mathscr{B}(G, L)$.

As we will see in section 6.3, the following theorem of Rapoport and Zink is one of the key ingredients for the proof of the representably of the Rapoport-Zink functor for local $\mathbb{P}$-shtukas by a formal scheme locally formally of finite type.

Theorem 6.1.3. (Rapoport-Zink) Let $b$ be an element of $G(L)$ which satisfies a decency equation relative to $s>0$. Then for any positive integer $r$ we have the following inclusion

$$
\{x \in \mathscr{B}(G, L) ; d(x, b \tilde{\sigma}(x))<r\} \subseteq \bigcup_{x_{0} \in \mathscr{B}\left(J, F_{s}\right)} B_{c}\left(x_{0}\right) .
$$

for some integer $c \gg 0$.

Proof. See [RZ2, theorem 1.4].

Remark 6.1.4. Note that the metric $d_{\mathscr{B}}$ on the Bruhat-Tits building of $G$ induces a metric on the $k$-valued points of $\widehat{\mathcal{F}}_{\mathbb{P}}$, which we denote by $d$. Recall that by proposition $4.1 .8, \widehat{\mathcal{F}} l_{\mathbb{P}}$ can be viewed as a parameter space for local $\mathbb{P}$-shtukas together with a quasi isogeny to a fixed one. For two such pairs $\underline{x}=(\underline{\mathcal{L}}, \bar{\delta})$ and $\underline{x}^{\prime}=\left(\underline{\mathcal{L}}^{\prime}, \bar{\delta}^{\prime}\right)$ over $k$, let $d\left(\underline{x}, \underline{x}^{\prime}\right)$ denote the distance between corresponding points of the affine flag variety $\mathcal{F} \ell_{\mathbb{P}}(k)$.

\subsection{The boundedness conditions}

In this section we first recall the notion of bounds on the isomorphisms of $L G$-torsors, introduced in $[\mathrm{H}-\mathrm{V}]$, where $G$ is a split reductive group over $\mathbb{F}_{q}$. Then we give an axiomatic definition of the boundedness condition for nonconstant case, namely for $\mathbb{P}$-shtukas. Consequently we introduce bounds on the moduli of local $\mathbb{P}$-shtukas and global $\mathfrak{G}$-shtukas.

\subsubsection{Bounds on the modui of local objects}

Let $\mathbb{G}$ be a constant reductive group over $\mathbb{D}$, i.e. $\mathbb{G}=G_{0} \times_{\mathbb{F}_{q}} \mathbb{D}$ where $G_{0}$ is a split reductive group over $\mathbb{F}_{q}$. Set $G:=\mathbb{G} \times \mathbb{D} \dot{\mathbb{D}}$.

Fix a borel subgroup $B \subset G_{0}$. Let $\bar{B} \subset G_{0}$ be the Borel subgroup opposite to our fixed $B$. For a dominant weight $\lambda$ of $G_{0}$ we let

$$
V(\lambda):=\left(\operatorname{Ind}_{\bar{B}}^{G_{0}}(-\lambda)_{\text {dom }}\right)^{\vee}
$$


be the Weyl module of $G_{0}$ with highest weight $\lambda$. It is a cyclic $G_{0}$-module generated by a $B$-stable line on which $B$ acts through $\lambda$. Any other such $G_{0^{-}}$ module is a quotient of $V(\lambda)$, see for example [Ja, II.2.13]. For a $L^{+} \mathbb{G}$-torsor $\mathcal{L}_{+}$on a scheme $S$ we denote by $\left(\mathcal{L}_{+}\right)_{\lambda}$ the fpqc-sheaf of $\mathcal{O}_{S} \llbracket z \rrbracket$-modules on $S$ associated with the presheaf

$$
Y \longmapsto\left(\mathcal{L}_{+}(Y) \times\left(V(\lambda) \otimes_{\mathbb{F}_{q}} \mathcal{O}_{S} \llbracket z \rrbracket(Y)\right)\right) / L^{+} \mathbb{G}(Y)
$$

This means in particular that if $S^{\prime} \rightarrow S$ is an étale covering trivializing $\mathcal{L}_{+}$and if $\alpha: \mathcal{L}_{+S^{\prime}} \simeq L^{+} \mathbb{G}_{S^{\prime}}$ is an isomorphism of $L^{+} \mathbb{G}$-torsors with $p_{2}^{*} \alpha \circ\left(p_{1}^{*} \alpha\right)^{-1}=g \in L^{+} \mathbb{G}\left(S^{\prime \prime}\right)$ on $S^{\prime \prime}=S^{\prime} \times_{S} S^{\prime}$ (with $p_{i}$ the projection onto the $i$-th factor) then

$\left(\mathcal{L}_{+}\right)_{\lambda}(Y) \cong\left\{v^{\prime} \in V(\lambda) \otimes_{\mathbb{F}_{q}} \mathcal{O}_{S} \llbracket z \rrbracket\left(Y \times_{S} S^{\prime}\right): p_{2}^{*} v^{\prime}=g \cdot p_{1}^{*} v^{\prime}\right.$ on $\left.Y \times_{S} S^{\prime \prime}\right\}$.

Note that the sheaf $\left(\mathcal{L}_{+}\right)_{\lambda}$ is locally free in the Zariski-topology on $S$.

Furthermore, if $\mathcal{L}_{+}$and $\mathcal{L}_{+}^{\prime}$ are $L^{+} \mathbb{G}$-torsors on $S$ and $\delta: \mathcal{L} \simeq \mathcal{L}^{\prime}$ is an isomorphism of the associated $L G$-torsors then $\delta$ induces an isomorphism of sheaves of $\mathcal{O}_{S}((z))$-modules

$$
\delta:\left(\mathcal{L}_{+}\right)_{\lambda} \otimes_{\mathcal{O}_{S} \llbracket z \rrbracket} \mathcal{O}_{S}((z)) \stackrel{\sim}{\longrightarrow}\left(\mathcal{L}_{+}^{\prime}\right)_{\lambda} \otimes_{\mathcal{O}_{S} \llbracket z \rrbracket} \mathcal{O}_{S}((z)) .
$$

Definition 6.2.1. Let $S$ be a connected scheme in $\mathcal{N} i l_{\mathbb{F}_{q} \llbracket \zeta \rrbracket}$ and let $\mu$ be a dominant coweight of $G_{0}$. Let either $\tilde{z}=z-\zeta$ or $\tilde{z}=z$.

(a) Let $\mathcal{L}_{+}$and $\mathcal{L}_{+}^{\prime}$ be $L^{+} \mathbb{G}$-torsors on $S$ and let $\delta: \mathcal{L} \simeq \mathcal{L}^{\prime}$ be an isomorphism of the associated $L G$-torsors. The isomorphism $\delta$ is bounded by $(\mu, \tilde{z})$ if for each dominant weight $\lambda$ of $G_{0}$

$$
\begin{aligned}
\delta\left(\left(\mathcal{L}_{+}\right)_{\lambda}\right) & \subset \tilde{z}^{-\left\langle(-\lambda)_{\mathrm{dom}}, \mu\right\rangle}\left(\mathcal{L}_{+}^{\prime}\right)_{\lambda} \subset\left(\mathcal{L}_{+}^{\prime}\right)_{\lambda} \otimes_{\mathcal{O}_{S} \llbracket z \rrbracket} \mathcal{O}_{S}((z))(6.2 \\
{[\mu] } & =\left[\mu_{\delta}(s)\right] \text { in } \pi_{1}\left(G_{0}\right) \text { for all } s \in S .
\end{aligned}
$$

(b) A local $\mathbb{G}$-shtuka $\left(\mathcal{L}_{+}, \varphi\right)$ over $S$ is bounded by $\mu$ if the isomorphism

$$
\varphi: \sigma^{*} \mathcal{L} \stackrel{\mathcal{L}}{\longrightarrow}
$$

is bounded by $(\mu, z-\zeta)$.

Proposition 6.2.2. Let $G$ be as above. Let $\mathcal{L}_{+}$and $\mathcal{L}_{+}^{\prime}$ be $L^{+} \mathbb{G}$-torsors on $S$ for a connected scheme $S \in \mathcal{N} i l p_{\mathbb{F}_{q} \llbracket \zeta \rrbracket}$. Let either $\tilde{z}=z-\zeta$ or $\tilde{z}=z$ and let $\delta: \mathcal{L} \sim \mathcal{L}^{\prime}$ be an isomorphism of the associated $L G$-torsors. Let $\mu$ be a dominant coweight of $G_{0}$ satisfying (6.2.2). 
(a) Then the condition that $\delta$ is bounded by $(\mu, \tilde{z})$ is representable by a finitely presented closed immersion into $S$.

(b) If $S$ is reduced then $\delta$ is bounded by $(\mu, \tilde{z})$ if and only if this holds for the pullback to every geometric point of $S$. By (a) it is even enough to consider the pullback to the generic points of $S$.

Proof. cf. [H-V, Lemma 3.10].

Proposition 6.2.3. Let $\mathbb{G}$ be as above. Let $\underline{\mathcal{L}}=\left(\mathcal{L}_{+}, \varphi\right)$ and $\underline{\mathcal{L}}^{\prime}=\left(\mathcal{L}_{+}^{\prime}, \varphi^{\prime}\right)$ be two bounded local $\mathbb{G}$-shtukas over a quasi-compact scheme $S \in \mathcal{N}$ ilp $\mathbb{F}_{q} \llbracket \zeta \rrbracket$ and let $i: \bar{S} \hookrightarrow S$ be a closed immersion defined by a sheaf of ideals $\mathcal{I}$ which is locally nilpotent. Then a quasi-isogeny $\bar{f}: \underline{\mathcal{L}}_{\bar{S}} \rightarrow \underline{\mathcal{L}}_{\bar{S}}^{\prime}$ is bounded by $(\mu, z)$ for some $\mu$ if and only if its lift over $S$ (see proposition 3.1.3) is bounded by $(\tilde{\mu}, z)$ for some $\tilde{\mu}$.

Proof. cf. [H-V, Proposition 3.9].

Now we want to consider the general case. Suppose that $G$ is a reductive group over $\dot{\mathbb{D}}$.

Let us begin by recalling some facts about affine Weyl groups (cf. [H-R]). Let $S$ be a maximal split torus in $G$ and let $T$ be its centralizer. Since $k$ is algebraically closed, $G$ is quasi-split and so $T$ is a maximal torus in $G$. Let $N=N(T)$ be the normalizer of $T$. Consider the following short exact sequence

$$
0 \rightarrow T(L)_{1} \rightarrow T(L) \stackrel{\kappa_{T}}{\longrightarrow} X_{*}(T)_{I} \rightarrow 0,
$$

where $T(L)_{1}$ denotes the kernel of the Kottwitz homomorphism $\kappa_{T}$, see [Ko2, Section 7]. It turns out that $T(L)_{1}$ equals $\mathcal{T}^{0}(\mathbb{D})$ where $\mathcal{T}^{0}$ is the identity component of the Néron model of $T$.

The Iwahori-Weyl group associated to $S$ is the quotient group $\widetilde{W}=$ $N(L) / \mathcal{T}^{0}(\mathbb{D})$. The Iwahori-Weyl group $\widetilde{W}$ is an extension of the relative Weyl group $W_{0}=N(L) / T(L)$ by $X_{*}(T)_{I}$ :

$$
0 \rightarrow X_{*}(T)_{I} \rightarrow \widetilde{W} \rightarrow W_{0} \rightarrow 1
$$

Note that the group $\widetilde{W}$ is endowed with the structure of a quasi Coxetersystem, which may thus be equipped with a Bruhat-Chevalley (partial) order $\preceq$ and a length function $\ell$. 
Proposition 6.2.4. Let $\mathcal{I}$ be the Iwahori subgroup of $G(L)$ associated to an alcove contained in the apartment associated to the maximal split torus $S$. Then we have the Cartan decomposition for the loop group

$$
L G(\bar{k})=\mathcal{I}(\bar{k}) \cdot N(L) \cdot \mathcal{I}(\bar{k})
$$

and the map $\mathcal{I}$.n.I $\mapsto n \in \widetilde{W}$ induces a bijection

$$
\mathcal{I} \backslash G(K) / \mathcal{I} \sim \widetilde{\sim} \widetilde{W}
$$

If $P$ and $Q$ are two parahoric subgroups of $G(L)$ then we have a bijection

$$
Q \backslash G(L) / P \stackrel{\sim}{\rightarrow} \widetilde{W^{Q}} \backslash \widetilde{W} / \widetilde{W^{P}}
$$

where $\widetilde{W}^{P}:=(N(L) \cap P) / T(L)_{1}$.

Proof. Cf. [H-R, Prop 8].

Let $K$ be a maximal parahoric subgroup of $G(L)$. The subgroup $\widetilde{W}^{K}$ projects isomorphically to the factor group $W_{0}$, and the exact sequence presents $\widetilde{W}$ as a semi-direct product

$$
\widetilde{W}=X_{*}(T)_{I} \rtimes W_{0} .
$$

The Schubert variety $\mathcal{S}(\omega)$ associated to $\omega \in \widetilde{W} \backslash \widetilde{W} / \widetilde{W}^{P}$ is the indscheme theoretic closure of the $L^{+} \mathbb{P}$-orbit of $\omega$ in $\mathcal{F} \ell_{\mathbb{P}}$. It is a projective variety over $k$. For further details see [PR1] and [Ri].

Proposition 6.2.5. Assume that the group $G$ is absolutely simple, simply connected and splits over a tamely ramified extension of $L$, then the indscheme $\tilde{\mathcal{F}}:=\lim (\mathcal{S}(\omega)$ ) (the direct limit is taken with respect to the Bruhat order on $\widetilde{W})$, coincides with the ind-scheme $\mathcal{F} \ell_{\mathbb{P}}$.

Proof. cf. [PR1, Proposition 9.8].

Definition 6.2.6. A closed ind-subscheme $\hat{Z}$ of $\widehat{\mathcal{F}}_{\mathbb{P}}$ which is stable under the left $L^{+} \mathbb{P}$-action, such that $Z:=\hat{Z} \times{ }_{\operatorname{Spf} k \llbracket \zeta \rrbracket} \operatorname{Spec} k$ is a quasi-compact subscheme of $\mathcal{F} \ell_{\mathbb{P}}$ is called a bound.

Let $\mathcal{L}_{+}$and $\mathcal{L}_{+}^{\prime}$ be $L^{+} \mathbb{P}$-torsors on $S \in \mathcal{N} i l p_{k \llbracket \zeta \rrbracket}$ and let $\delta: \mathcal{L} \sim \mathcal{L}^{\prime}$ be

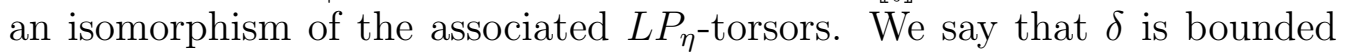
by $\hat{Z}$ if for any trivialization $S^{\prime} \rightarrow S$ of $\mathcal{L}_{+}$and $\mathcal{L}_{+}^{\prime}$ the induced morphism

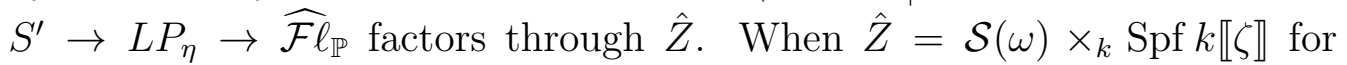
a Schubert variety $\mathcal{S}(\omega)$ (with $\omega \in \widetilde{W}$ ) we say that $\delta$ is bounded by $\omega$. Furthermore we say that a local $\mathbb{P}$-shtuka $\left(\mathcal{L}_{+}, \varphi\right)$ is bounded by $\hat{Z}$ if the isomorphism $\varphi$ is bounded by $\hat{Z}$. 
54CHAPTER 6. THE RAPOPORT-ZINK SPACE FOR LOCAL P-SHTUKAS

The closedness of the above boundedness condition is almost tautological (compare proposition 6.2.2).

The following crucial observation enables one to pull back bounds regarding certain embeddings of the group $\mathbb{P}_{1}$ into $\mathbb{P}_{2}$, see also proposition 4.1.3.

Proposition 6.2.7. Let $\mathbb{P}_{1} \subseteq \mathbb{P}_{2}$ be a closed embedding of smooth group schemes of finite type over $\mathbb{D}$ such that $\mathbb{P}_{1} \backslash \mathbb{P}_{2}$ is quasi-affine. Assume that $L P_{2, \eta} \rightarrow \mathcal{F} \ell_{\mathbb{P}_{2}}$ admits sections locally in the Zariski topology. Then $\mathcal{F} \ell_{\mathbb{P}_{1}} \rightarrow$ $\mathcal{F} \ell_{\mathbb{P}_{2}}$ is a locally closed embedding, and $L P_{1, \eta} \rightarrow \mathcal{F} \ell_{\mathbb{P}_{1}}$ admits sections locally in the étale topology. In addition, if $\mathbb{P}_{1} \backslash \mathbb{P}_{2}$ is affine, then $\mathcal{F} \ell_{\mathbb{P}_{1}} \rightarrow \mathcal{F} \ell_{\mathbb{P}_{2}}$ is a closed embedding.

Proof. cf. [BD, Section 4.5].

Example 6.2.8. Assume that $\operatorname{char} \mathbb{F}_{q} \neq 2$. Set $K:=\mathbb{F}_{q}((z))$. Let $E:=$ $K(y)$ be a quadratic field extension, say $y^{2}=z$. Let $T$ be the one dimensional torus $\operatorname{ker}\left(N_{E / K}: \operatorname{Res}_{E / K} \mathbb{G}_{m} \rightarrow \mathbb{G}_{m}\right)$. Explicitly $T=\operatorname{Spec} K[a, b] /\left(a^{2}-\right.$ $\left.b^{2} z-1\right)$, with the multiplication $(a, b) *(c, d)=(a c+b d z, a d+b c)$. Sending $a \mapsto \frac{1}{2}\left(t+t^{-1}\right)$ and $b \mapsto \frac{1}{2 y}\left(t^{-1}-t\right)$ defines an isomorphism $\mathbb{G}_{m, E}=$ Spec $E\left[t, t^{-1}\right] \cong T_{E}$ which we will use in the sequel to identify $X_{*}\left(T_{E}\right)$ with $\mathbb{Z}$. The inertia group $I=\operatorname{Gal}(E / K)=\{1, \gamma\}$ acts on $X_{*}(T)=\mathbb{Z}$ via $\gamma(\lambda)=-\lambda$ and hence $X_{*}(T)_{I}=\mathbb{Z} / 2 \mathbb{Z}$.

With each element of $X_{*}(T)_{I}$ the inverse of the Kottwitz map associates a $\hat{\sigma}$-conjugacy class in $\operatorname{LT}\left(\mathbb{F}_{q}^{\text {sep }}\right)$. For example for $\bar{\mu}=1 \in X_{*}(T)_{I}=\mathbb{Z} / 2 \mathbb{Z}$ one has to choose a lift $\mu \in X_{*}\left(T_{E}\right)$. If we choose $\mu=1$ then with $\bar{\mu}=1$ it associates

$$
\begin{aligned}
N_{E / K}(\mu(y)) & =\mu(y) \cdot \gamma(\mu(y)) \\
& =\left(\frac{1}{2}\left(y+y^{-1}\right), \frac{1}{2 y}\left(y^{-1}-y\right)\right) \cdot\left(\frac{1}{2}\left(-y-y^{-1}\right), \frac{1}{-2 y}\left(-y^{-1}-(-y)\right)\right) \\
& =(-1,0) \in T\left(\mathbb{F}_{q}^{\text {sep }}((z))\right) .
\end{aligned}
$$

This is independent of the choice of $\mu$ and of the uniformizer $y$ (and of $E$ ).

Consider the Néron-model $\mathcal{T}=\operatorname{ker}\left(N_{\mathcal{O}_{E} / \mathcal{O}_{K}}: \operatorname{Res}_{\mathcal{O}_{E} / \mathcal{O}_{K}} \mathbb{G}_{m} \rightarrow \mathbb{G}_{m}\right)$, as a scheme it is isomorphic to Spec $\frac{\mathbb{F}_{q} \llbracket z \rrbracket[a, b]}{a^{2}-b^{2} z-1}$. Its special fiber has two connected components distinguished by $a \equiv 1$ or $-1(\bmod z)$. Therefore the connected component of identity of $\mathcal{T}$ is $\mathcal{T}^{0}:=\operatorname{Spec} \mathbb{F}_{q} \llbracket z \rrbracket\left[a^{\prime}, b\right] /\left(2 a^{\prime}+\right.$ $\left.z\left(a^{\prime}\right)^{2}-b^{2}\right)$, where $a=1+z a^{\prime}$. In particular $(-1,0) \notin \mathcal{T}^{0}\left(\mathbb{F}_{q}((z))\right)$.

Now consider the local $L^{+} \mathcal{T}^{0}$-shtuka $\left(\left(L^{+} \mathcal{T}^{0}\right)_{\mathbb{F}_{q}},(-1,0)\right)$ over $\mathbb{F}_{q}$ which is bounded by $1 \in \widetilde{W}=X_{*}(T)_{I}$. We want to lift it to a local $L^{+} \mathcal{T}^{0}$-shtuka over $\mathbb{F}_{q} \llbracket \zeta \rrbracket[\eta] /\left(\eta^{2}-\zeta\right)$ 
Consider the isomorphism $K=\mathbb{F}_{q}((z)) \rightarrow \mathbb{F}_{q}((\zeta)), z \mapsto \zeta$. Fix an embedding $i: E \hookrightarrow \mathbb{F}_{q}((\zeta))^{\text {alg }}$ and let $\mu=1 \in \mathbb{Z}=X_{*}\left(T_{E}\right)$. Set $\eta:=i(y)$ and lift $N_{E / K}(\mu(y))$ to $g(E, \mu, i):=N_{E / K}(\mu(y-i(y)))$. We compute

$$
\begin{gathered}
(\alpha, \beta):=\mu(y-\eta) \cdot \gamma(\mu(y-\eta))= \\
\left(\frac{1}{2}\left((y-\eta)+(y-\eta)^{-1}\right), \frac{1}{2 y}\left((y-\eta)^{-1}-(y-\eta)\right)\right) \\
\cdot \gamma\left(\frac{1}{2}\left((y-\eta)+(y-\eta)^{-1}\right), \frac{1}{2 y}\left((y-\eta)^{-1}-(y-\eta)\right)\right),
\end{gathered}
$$

then

$$
\begin{gathered}
\alpha=\frac{1}{4}\left((y-\eta)+(y-\eta)^{-1}\right)\left((-y-\eta)+(-y-\eta)^{-1}\right) \\
-\frac{1}{4}\left((y-\eta)^{-1}-(y-\eta)\right)\left((-y-\eta)^{-1}-(-y-\eta)\right) \\
=\frac{1}{2}\left(\frac{(y-\eta)^{2}+(-y-\eta)^{2}}{(-y-\eta)(y-\eta)}\right) \\
=\frac{\zeta+z}{\zeta-z},
\end{gathered}
$$

and

$$
\begin{gathered}
\beta=\frac{-1}{4 y}\left((y-\eta)+(y-\eta)^{-1}\right)\left((-y-\eta)^{-1}-(-y-\eta)\right) \\
+\frac{1}{4 y}\left((y-\eta)^{-1}-(y-\eta)\right)\left((-y-\eta)+(-y-\eta)^{-1}\right) \\
=\frac{2 \eta}{\zeta-z} .
\end{gathered}
$$

Thus we get

$$
g(E, i):=g(E, 1, i)=N_{E / K}(\mu(y-\eta))=\left(\frac{\zeta+z}{\zeta-z}, \frac{2 \eta}{\zeta-z}\right) .
$$

This shows that we can lift the local shtuka to a local shtuka over $\mathbb{F}_{q} \llbracket \eta \rrbracket$.

However the lift depends on the choice of $\mu$ and of the embedding $i$.

We first compute how $g(E, i):=N_{E / K}(\mu(y-i(y)))$ depends on the chosen embedding $i$ and compute $\frac{N_{E / K}(\mu(y-i(y)))}{N_{E / K}(\mu(y-i \circ \gamma(y))}$. Changing $i$ replaces $\eta$ by $-\eta$ and we have

$$
g(E, i \circ \gamma)=N_{E / K}\left(\mu(y-\gamma(\eta))=\left(\frac{\zeta+z}{\zeta-z}, \frac{-2 \eta}{\zeta-z}\right) .\right.
$$

Note that $g(E, i)=g(E, i \circ \gamma)^{-1}$. Hence 


$$
\frac{g(E, i)}{g(E, i \circ \gamma)}=g(E, i)^{2}=\left(\frac{(z+\zeta)^{2}+4 \zeta z}{(\zeta-z)^{2}}, \frac{4 \eta(z+\zeta)}{(\zeta-z)^{2}}\right) .
$$

This also shows that what happens if we replace $\mu \in X_{*}\left(T_{E}\right)=\mathbb{Z}$ by another lift of $\bar{\mu} \in \mathbb{Z} / 2 \mathbb{Z}$, i.e.

$$
\frac{g(E, \mu+2, i)}{g(E, \mu, i)}=g(E, 2, i)=g(E, 1, i)^{2} .
$$

Observe that $\frac{g(E, i)}{g(E, i \circ \gamma)} \in \mathcal{T}^{0}\left(\mathbb{F}_{q} \llbracket z, \frac{\eta}{z} \rrbracket\right) \backslash \mathcal{T}^{0}\left(\mathbb{F}_{q} \llbracket \eta, z \rrbracket\right)$. So $g(E, \gamma \circ i)$ does not lie in the closure of the subscheme $L^{+} \mathcal{T}^{0} g(E, i) L^{+} \mathcal{T}^{0} \subset L \mathcal{T}^{0} \times_{\mathrm{Spec} \mathbb{F}_{q}}$ Spf $\mathbb{F}_{q} \llbracket \eta \rrbracket$.

\subsubsection{Bounds on the moduli of global objects}

The following theorem and lemma generalize [Beh, theorem 4.4.1].

Theorem 6.2.9. Let $X$ be a projective scheme over the field $k$. Let $\mathcal{V}$ be a vector bundle over $X$ and $\mathfrak{G} \hookrightarrow G l(\mathcal{V})$ a closed subgroup with quasi-affine quotient $G l(\mathcal{V}) / \mathfrak{G}$. Then the natural morphism of $k$-stacks

$$
\rho_{*}: \mathscr{H}^{1}(X, \mathfrak{G}) \rightarrow \mathscr{H}^{1}(X, G l(\mathcal{V}))
$$

is representable, quasi-affine and of finite presentation.

Proof. Let $p_{S}: X_{S} \rightarrow S$ be the projection map and view this as a morphism $E t\left(X_{S}\right) \rightarrow E t(S)$ of big étale sites. For any scheme $Y$ over $X_{S}$ let $p_{S *}(Y)$ denote the sheaf which sends a scheme $T$ to $\operatorname{Hom}_{X_{S}}\left(X_{T}, Y\right)$. Let $\mathcal{G}$ be a $G L(\mathcal{V})$-bundle in $\mathscr{H}^{1}(X, G L(\mathcal{V}))(S)$. We have the following 2-cartesian diagram of stacks

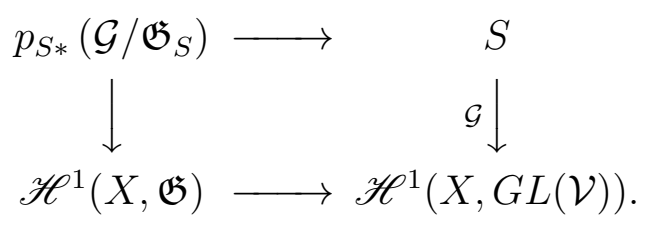

(Note that this follows from a more general fact that for a given monomorphism $G_{1} \rightarrow G_{2}$ of group schemes over a base scheme $\widetilde{X}$, the category of reductions of a $G_{2}$-bundle $\widetilde{\mathcal{G}}$ to $G_{1}$ (which is indeed a set, since $G_{1} \rightarrow G_{2}$ is is mono) equals the set of global sections of $\widetilde{\mathcal{G}} / G_{1}$, see [Beh, Prop. 4.2.3].)

Let $\mathcal{G}_{\text {univ }}$ be the universal $G L(\mathcal{V})$-bundle over $X \times \mathscr{H}^{1}(X, G L(\mathcal{V}))$. By the assumption $\mathcal{G}_{\text {univ }} / \mathfrak{G}$ is quasi-affine over $\mathscr{H}^{1}(X, \mathfrak{G})$. Thus the theorem follows from the lemma below. 
Lemma 6.2.10. Let $X$ be a projective scheme over the field $k$. Let $p$ denote the structure morphism $p: X \rightarrow$ Spec $k$. Let $S$ be a k-scheme and $\tilde{X} \rightarrow X_{S}$ a quasi-affine $X_{S}$-scheme of finite presentation. Then $p_{S_{*}} \tilde{X}$ is a quasi-affine $S$-scheme of finite presentation.

Remark 6.2.11. In [Beh, Prop 4.4.1 and 4.4.4], K. Behrend considers the case that $\tilde{X} \rightarrow X_{S}$ is affine and then proves that $p_{S *} \tilde{X}$ is an affine $S$-scheme of finite presentation. We prove bellow that the above lemma reduces to the case studied by K. Behrend.

Proof. Let $i: \tilde{X} \rightarrow Y$ be an open immersion of $\tilde{X}$ into an affine $X_{S}$-scheme $Y$. Set $\mathcal{A}:=p_{*} Y$. Consider the universal morphism $X_{\mathcal{A}} \rightarrow Y$ corresponding to the $i d \in \mathcal{A}(\mathcal{A})$. Let $Z \hookrightarrow X_{\mathcal{A}}$ be the complement of $X_{\mathcal{A}} \times_{Y} \tilde{X}$ in $X_{\mathcal{A}}$. By properness of $X, Z$ maps to a closed subscheme of $\mathcal{A}$. Let $\mathcal{U}$ denote the complement of $p(Z)$ in $\mathcal{A}$. We claim that $\mathcal{U}$ represents $p_{*} \tilde{X}$. To see this first observe that the open immersion $X_{\mathcal{U}} \hookrightarrow X_{\mathcal{A}} \times_{Y} \tilde{X}$ gives a morphism $X_{\mathcal{U}} \rightarrow \tilde{X}$ which induces a $\mathcal{U}$-point in $p_{*} \tilde{X}(\mathcal{U})$. Hence it is enough to check that for any scheme $T$ we have the inclusion $p_{*}(\tilde{X})(T) \subseteq \mathcal{U}(T)$. Any point of $p_{*} \tilde{X}(T)$ is a morphism $X_{T} \rightarrow \tilde{X}$, composing with $i: \tilde{X} \rightarrow Y$ induces a $T$ valued point $\alpha$ of $\mathcal{A}$. We have to show that $\alpha: T \rightarrow \mathcal{A}$ factors through the open subscheme $\mathcal{U}$. One can easily check this in the level of the topological spaces. Namely, if $T \times_{\mathcal{A}} p(Z)$ is non-empty then so is $X_{T} \times_{X_{\mathcal{A}}} Z$, which is a contradiction, since $Z$ is defined as the complement of $X_{\mathcal{A}} \times_{Y} \tilde{X}$ in $X_{\mathcal{A}}$. Thus the proof of the theorem reduces to the case that $\tilde{X}$ is affine over $X_{S}$. This then follows from remark 6.2.11.

Theorem 6.2.12. The stack $\mathscr{H}^{1}(C, \mathfrak{G})$ is a smooth algebraic $k$-stack, which is locally of finite type.

Proof. This is well-known for $\mathfrak{G}=G l_{n}$. Thus $\mathscr{H}^{1}(X, \mathfrak{G})$ is locally of finite type by 4.1 .3 and 6.2.9. The smoothness follows from the vanishing of the second cohomology of coherent sheaves on a curve.

Remark 6.2.13. Consider the following functor

$$
\begin{aligned}
S \longmapsto & \left\{\left(\mathcal{G}, s_{1} \ldots, s_{n}, \varphi\right) ; \mathcal{G} \text { is a G-bundle over } C_{S}, s_{i} \in C(S)\right. \\
& \left.\varphi:\left.\mathcal{G}\right|_{C_{S} \backslash \cup_{i} \Gamma_{s_{i}}} \rightarrow \mathfrak{G} \times{ }_{C} C_{S} \backslash \cup_{i} \Gamma_{s_{i}} \text { is a trivializaition }\right\} .
\end{aligned}
$$

This functor is representable by an ind-scheme $G r_{\mathfrak{G}, n}$ over $C^{n}$ locally of finite type, see [Ga]. The ind-scheme $G r_{\mathfrak{G}, n}$ is called global affine grassmannian. 
Remark 6.2.14. In order to obtain an algebraic substack (of finite type) of Hecke $_{\mathfrak{G}}$ one has to control the relative position of $\varphi: \mathcal{G} \sim \mathcal{G}^{\prime}$ in the moduli stack Hecke $_{\mathfrak{G}}$. Here we recall the boundedness condition introduced in [Var], when the group $\mathfrak{G}$ is constant, i.e. it comes from a split connected reductive group $G_{0}$ over $\mathbb{F}_{q}$ by base change. For a dominant weight $\lambda$ of $G_{0}$, consider the representation $\rho_{\lambda}: G_{0} \rightarrow G l\left(V_{\lambda}\right)$, where $V_{\lambda}$ denote the Weyl module of $G_{0}$ with the highest weight $\lambda$. This representation induces a 1-morphsim $\rho_{\lambda_{*}}: \mathscr{H}^{1}(C, \mathfrak{G}) \rightarrow \mathscr{H}^{1}\left(C, G l\left(V_{\lambda}\right)\right)$. For a $\mathfrak{G}$-bundle $\mathcal{G}$ we let $\mathcal{G}_{\lambda}$ denote the vector bundle associated to the $G l\left(V_{\lambda}\right)$-bundle $\rho_{\lambda_{*}} \mathcal{G}$. Let $\varphi:\left.\left.\mathcal{G}\right|_{C_{S} \backslash \cup \Gamma_{s_{i}}} \rightarrow \mathcal{G}^{\prime}\right|_{C_{S} \backslash \cup \Gamma_{s_{i}}}$ be an isomorphism between $\mathfrak{G}$-torsors over $C_{S}$ outside (the graphs of) the characteristic sections. Then $\varphi$ is said to be bounded by an $n$-tuple of dominant coweights $\underline{\omega}=\left(\omega_{1}, \ldots, \omega_{n}\right)$ of $G_{0}$ if

i) $\varphi\left(\mathcal{G}_{\lambda}\right) \subseteq \mathcal{G}_{\lambda}^{\prime}\left(\sum_{\lambda}\left\langle\lambda, \omega_{i}\right\rangle \Gamma_{s_{i}}\right)$ for each dominant weight $\lambda$ of $G_{0}$;

ii) $\pi_{0}\left(\mathcal{G}_{s}\right)-\pi_{0}\left(\mathcal{G}_{s}^{\prime}\right)=\sum_{i}\left[\omega_{i}\right]$ for each geometric point $s \in S$.

Let $H e c k e_{\mathfrak{G}, D, n}^{\omega}$ denote the closed substack of $H e c k e_{\mathfrak{G}, D, n}$ defined by imposing the above conditions on the universal isomorphism $\varphi_{\text {univ }}$ on $H e c k e_{\mathfrak{G}, D, n}$.

Fix a faithful representation $\rho: \mathfrak{G} \rightarrow G l\left(\mathcal{V}_{0}\right)$, for some vector bundle $\mathcal{V}_{0}$ of rank $r$, with quasi-affine quotient $G l\left(\mathcal{V}_{0}\right) / \mathfrak{G}$ (see proposition 4.1.3) and consider the induced morphism

$$
\rho_{*}: \mathscr{H}^{1}(C, \mathfrak{G}) \rightarrow \mathscr{H}^{1}\left(C, G l\left(\mathcal{V}_{0}\right)\right) \simeq V e c t_{C}^{r}
$$

of stacks. Here $V e c t_{C}^{r}$ is the stack whose $S$-valued points parametrizes rank $r$ vector bundles over $C_{S}=C \times_{k} S$.

Let $\underline{\omega}:=\left(\omega_{i}\right)$ be an $n$-tuple of dominant coweights of $G l_{n}$. Consider the relative affine grassmanninan $\mathcal{G} r_{\mathfrak{G}, n}$ over $C^{n} \times \mathscr{H}^{1}(C, \mathfrak{G})$ which parametrizes tuples $\left(\mathcal{G}, \mathcal{V}^{\prime}, s_{1}, \ldots s_{n}, \varphi\right)$, where

$$
\left(\mathcal{G}, \mathcal{V}^{\prime}, s_{1}, \ldots s_{n}\right) \in \mathscr{H}^{1}(C, \mathfrak{G}) \times \mathscr{H}^{1}\left(C, G l\left(\mathcal{V}_{0}\right)\right) \times C^{n}
$$

and $\varphi$ is an isomorphism between the vector bundle associated to $\rho_{*} \mathcal{G}$ and $\mathcal{V}^{\prime}$ outside the graphs $\cup_{i} \Gamma_{s_{i}}$. Note that the morphism $\rho_{*}$ yields a morphism Hecke $_{\mathfrak{G}, n} \rightarrow \mathcal{G} r_{\mathfrak{G}, n}$, which sends $\left(\mathcal{G}, \mathcal{G}^{\prime},\left(s_{i}\right)_{i}\right)$ to $\left(\mathcal{G}, \rho_{*} \mathcal{G}^{\prime},\left(s_{i}\right)_{i}\right)$.

Now let $\mathcal{G} r_{\mathfrak{G}, n}^{\omega}$ denote the substack of $\mathcal{G} r_{\mathfrak{G}, n}$ defined by the condition that the universal isomorphism $\varphi_{\text {univ }}$ is bounded by $\underline{\omega}$.

Remark 6.2.15. Note that the relative affine Grassmannian $\mathcal{G} r_{\mathfrak{G}, n}^{\omega}$ is projective over $\mathscr{H}^{1}(C, \mathfrak{G})$. To see this (since $C$ is projective) we may look at the fiber of $\mathcal{G} r_{\mathfrak{G}, n}^{\omega} \rightarrow \mathscr{H}^{1}(C, \mathfrak{G}) \times_{\mathbb{F}_{q}} C^{n}$ over $\left(\mathcal{G}, s_{1}, \ldots, s_{n}\right) \in \mathscr{H}^{1}(C, \mathfrak{G}) \times_{\mathbb{F}_{q}}$ $C^{n}(S)$, and further notice that bounding $\varphi$ by $\omega$ is equivalent to bounding $\varphi^{-1}$ by $(-\omega)_{\text {dom }}$ which is representable by a projective variety. 
The stack Hecke $\frac{\omega}{\mathfrak{G}, n}$ is defined by the following pull-back diagram

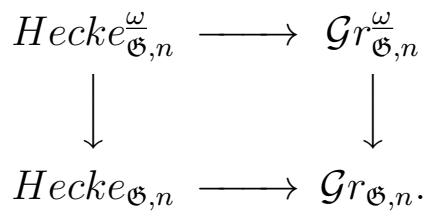

Proposition 6.2.16. Let $\rho: \mathfrak{G} \rightarrow G l\left(\mathcal{V}_{0}\right)$ be a faithful representation as above with quasi-affine (resp. affine) quotient $G l\left(\mathcal{V}_{0}\right) / \mathfrak{G}$. The stack Hecke $_{\mathfrak{G}}$ is represented by a locally closed (resp. closed) substack of $\mathcal{G} r_{\mathfrak{G}, n}$. In particular the stack Hecke $e_{\mathfrak{G}, D, n}$ is quasi-projective (resp. projective) over $\mathscr{H}^{1}(C, \mathfrak{G})$.

Proof. The restriction of the triple $\left(\mathcal{G}_{\text {univ }}, \mathcal{V}_{\text {univ }}^{\prime}, \varphi_{\text {univ }}\right)$ to $U$, the complement of the graphs $\Gamma_{s_{i}}$ in $C \times \mathcal{G} r_{\mathfrak{G}}$, defines a section $s \in\left(\mathcal{G}_{\text {univ }} / \mathfrak{G}\right)(U)$, see theorem 6.2.9. Then since $\mathcal{G}_{\text {univ }} / \mathfrak{G}$ is quasi-affine over $C \times \mathcal{G} r_{\mathfrak{G}}$, thus by the following lemma, there exists a locally closed substack $\mathcal{H}$ of $\mathcal{G} r_{\mathfrak{G}}$ such that $s$ extends over $\mathcal{H} \times C$ and by definition this substack represents $H_{e c k e} e_{\mathfrak{G}, n}$. Finally the last statement of the proposition follows from the fact that $\mathcal{G} r \frac{\omega}{\mathfrak{G}, n}^{n}$ is projective over $\mathscr{H}^{1}(C, \mathfrak{G})$.

Lemma 6.2.17. Let $\mathcal{Y}$ be a quasi-affine scheme over $X \times S$. Let $U$ be the complement of the graph of the section $s \in X(S)$. Let $t: U \rightarrow \mathcal{Y}$ be a section. Then the question whether $t$ extends to $X \times S \rightarrow \mathcal{Y}$ is representable by a locally closed subscheme of $S$.

Proof. cf. [Ga, A.5].

We denote by $\nabla \frac{\omega}{n} \mathscr{H}_{D}^{1}(C, \mathfrak{G})$ the pull back of $H e c k e^{\frac{\omega}{\mathfrak{G}, D, n}}$ under the closed immersion $\nabla_{n} \mathscr{H}^{1}(C, \mathfrak{G}) \hookrightarrow$ Hecke $_{\mathfrak{G}, D, n}$. Similarly we define

$$
\nabla_{n}^{H, \underline{\omega}} \mathscr{H}_{D}^{1}(C, \mathfrak{G}):=\nabla_{n}^{H} \mathscr{H}^{1}(C, \mathfrak{G}) \times_{\nabla \mathscr{H}^{1}(C, \mathfrak{G})} \nabla_{n}^{\underline{\omega}} \mathscr{H}_{D}^{1}(C, \mathfrak{G})
$$

Proposition 6.2.18. Let $D$ be a finite subscheme of $C$ and let $H_{D}$ denote the associated level structure, see remark 5.2.4. The stack $\nabla \frac{\omega}{n} \mathscr{H}_{D}^{1}(C, \mathfrak{G})$ is an Artin stack over $(C \backslash D)^{n}$ locally of finite type. Furthermore, let $\underline{\nu}=\left(\nu_{i}\right)$ be an $n$-tuple of places of $C$, for a compact open subgroup $H \subseteq$ $\mathfrak{G}\left(\mathbb{A} \frac{\nu}{Q}\right)$ the stack $\nabla_{n}^{H, \underline{\omega}} \mathscr{H}^{1}(C, \mathfrak{G})^{\underline{\nu}}$ is a formal algebraic stack over $\prod_{i} \operatorname{Spf} A_{\nu_{i}}$ locally of finite type. In particular $\nabla_{n} \mathscr{H}_{D}^{1}(C, \mathfrak{G}) \quad\left(\right.$ resp. $\left.\nabla_{n}^{H} \mathscr{H}^{1}(C, \mathfrak{G})^{\underline{\nu}}\right)$ is an ind-algebraic stack ind-quasi-projective over $C^{n} \times_{\mathbb{F}_{q}} \mathscr{H}^{1}(C, \mathfrak{G})$ (resp. $\left.\prod_{i} \operatorname{Spf} A_{\nu_{i}} \times_{\mathbb{F}_{q}} \mathscr{H}^{1}(C, \mathfrak{G})\right)$. 
60CHAPTER 6. THE RAPOPORT-ZINK SPACE FOR LOCAL P-SHTUKAS

Proof. By Lang's theorem the stack $\nabla \mathscr{H}^{1}\left(\operatorname{Supp} D, \mathfrak{G}_{D}\right)$ is isomorphic to the classifying stack $\mathscr{H}^{1}\left(\mathbb{F}_{q}, \mathfrak{G}_{D}\right)$. Thus one obtains the following 2-cartesian diagram

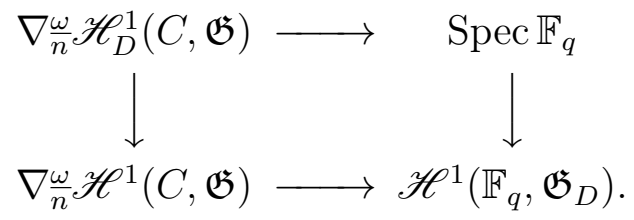

We observe that $\nabla_{\bar{n}}^{\omega} \mathscr{H}_{D}^{1}(C, \mathfrak{G}) \rightarrow \nabla_{\bar{n}}^{\omega} \mathscr{H}^{1}(C, \mathfrak{G})$ is a principal $\mathfrak{G}_{D}$ bundle. The stack $\nabla_{n}^{H_{D}} \mathscr{H}^{1}(C, \mathfrak{G})$ is a closed substack of Hecke $\mathfrak{G}_{, D, n}$. Recall that by proposition 6.2.16 the stack $H e c k e e_{\mathfrak{G}, n}^{\omega}$ is quasi projective over $\mathscr{H}^{1}(C, \mathfrak{G})$. Hence the statement follows from the corresponding fact about $\mathscr{H}^{1}(C, \mathfrak{G})$. For the second part of the theorem, first observe that conjugating the level structure $H$ with $g \in \mathfrak{G}\left(\mathbb{A} \frac{\nu}{Q}\right)$ induces an isomorphism

$$
\nabla_{n}^{H, \underline{\omega}} \mathscr{H}^{1}(C, \mathfrak{G})^{\underline{\nu}} \stackrel{\sim}{\rightarrow} \nabla_{n}^{g H g^{-1}, \underline{\omega}} \mathscr{H}^{1}(C, \mathfrak{G})^{\underline{\nu}}
$$

and hence (after conjugating the level structure with a sutable $g$ ) we may assume that $H \subseteq \mathfrak{G}\left(\mathbb{A}^{\underline{\nu}}\right)$. Since $H$ is open we may take a closed subscheme $D \subseteq C \backslash\left\{\nu_{i}\right\}$ such that

$$
H \supseteq H_{D}:=\operatorname{ker}\left(\mathfrak{G}\left(\mathbb{A}^{\underline{\nu}}\right) \rightarrow \mathfrak{G}\left(\mathcal{O}_{D}\right)\right),
$$

and thus reduce to the case that the level structure $H$ is of the form $H_{D}$, where $D$ is supported outside the places $\nu_{i}$ by proposition 5.2.6.

The last statement follows from remark 6.2.15 and proposition 6.2.16.

Remark 6.2.19. One can observe that $\mathscr{H}^{1}(C, \mathfrak{G})$ admits a covering $\left\{U_{\alpha}\right\}_{\alpha}$ by connected open substacks of finite presentation over $k$. This is well known when $\mathfrak{G}$ is a constant split reductive group over $\mathbb{F}_{q}$. In this case the index set is $\{(\mu, c)\}$ where $\mu$ runs over the cocharacters of $G$ and $c \in \pi_{1}(G)$. To see this for the general $\mathfrak{G}$ one may use theorem 6.2.9.

For sufficiently small $H=H_{D} \subseteq \mathfrak{G}\left(\mathbb{A}^{\underline{\nu}}\right)$ the restriction $\nabla_{n}^{H} \mathscr{H}_{\alpha}^{1}(C, \mathfrak{G})$ of the stack $\nabla_{n}^{H} \mathscr{H}^{1}(C, \mathfrak{G})$ to $U_{\alpha}$ is a quotient of a quasi-projective scheme $X_{\alpha, H, \underline{\omega}}$ over $(C \backslash D)^{n}$ by a finite group $G_{H_{D}}$, see [Var, Prop 2.16].

One can also bound the moduli stack of global $\mathfrak{G}$-shtukas by more intrinsic bounds, namely by those coming from local objects, i.e. 
Definition 6.2.20. Let $\mathbb{P}_{\nu}$ denote the completion of $\mathfrak{G}$ at the place $\nu$ on $C$. Fix an n-tuple $\underline{\nu}=\left(\nu_{i}\right)$ of places on the curve $C$. Let $\widehat{Z}_{\underline{\nu}}:=\left(\widehat{Z}_{\nu_{i}}\right)_{i}$ be a tuple of closed subschemes $\widehat{Z}_{\nu_{i}}$ of $\widehat{\mathcal{F}} \ell_{\mathbb{P}_{\nu_{i}}}$ subject to conditions a), b)and c) of definition 6.2.6. Let $\underline{\mathcal{G}}$ be a global $\mathfrak{G}$-shtuka in $\nabla_{n} \mathscr{H}^{1}(C, \mathfrak{G})^{\overline{\underline{\nu}}}(S)$. We say that $\underline{\mathcal{G}}$ is bounded by $\widehat{\widehat{Z}}_{\underline{\nu}}:=\left(\widehat{Z}_{\nu_{i}}\right)_{i}$ if for any $i$ the associated local $\mathbb{P}_{\nu_{i}}$-shtuka $\widehat{\mathcal{G}}_{\nu_{i}}$ is bounded by $\widehat{Z}_{\nu_{i}}$. We denote by $\nabla_{n}^{\widehat{Z}_{\underline{\mu}}} \mathscr{H}^{1}(C, \mathfrak{G})^{\underline{\underline{\nu}}}(S)$ the substack of $\nabla_{n} \mathscr{H}^{1}(C, \mathfrak{G})^{\underline{\underline{\nu}}}(S)$ consisting of global $\mathfrak{G}$-shtukas bounded by $\widehat{Z}_{\underline{\underline{\nu}}}$.

\subsection{Representablity of The Rapoport-Zink Functor}

Let $G$ denote the generic fiber of $\mathbb{P}$ and let $\bar{b}$ be an element of $L G(k)$.

Let $\hat{Z} \subset \widehat{\mathcal{F}} \ell_{\mathbb{P}}$ be a bound, see definition 6.2.6, and set $Z=\hat{Z} \times_{\text {Spf } \mathbb{F}} \llbracket \llbracket \rrbracket$ Spec $\mathbb{F}_{q}$. We define the associated affine Deligne-Lusztig variety

$$
X_{Z}(b)=\left\{g \in \mathcal{F} \ell(k) ; g^{-1} \bar{b} \sigma^{*} g \in Z(k)\right\} .
$$

For $\omega \in \widetilde{W}$ we set $X_{\omega}(b):=X_{\mathcal{S}(\omega)}(b)$.

In the remaining part of the chapter we show the pro-representablity of the Rapoport-Zink space for local $\mathbb{P}$-shtukas. Let $\underline{\mathbb{L}}_{0}$ be as before (see proposition 4.1.8).

Consider the functor

$$
\begin{aligned}
& \mathcal{M}_{\underline{\mathcal{L}}_{0}}^{\hat{\mathcal{L}}}:\left(\mathcal{N} i l p_{k \llbracket \zeta \rrbracket}\right)^{o} \rightarrow \mathcal{S e t s} \\
& S \longmapsto\left\{(\underline{\mathcal{L}}, \bar{\delta}) ; \underline{\mathcal{L}} \in O b\left(\underline{\mathcal{M}}_{\underline{\mathbb{L}}_{0}}(S)\right) \text { is bounded by } \hat{Z}\right. \text { and } \\
&\left.\bar{\delta}: \underline{\mathcal{L}}_{\bar{S}} \rightarrow \underline{\mathbb{L}}_{0, \bar{S}} \text { is a quasi-isogeny }\right\} / \sim .
\end{aligned}
$$

Here $(\underline{\mathcal{L}}, \bar{\delta})$ and $\left(\underline{\mathcal{L}}^{\prime}, \bar{\delta}^{\prime}\right)$ are called isomorphic if $\bar{\delta}^{-1} \circ \bar{\delta}^{\prime}$ lifts to an isomorphism $\underline{\mathcal{L}}^{\prime} \rightarrow \underline{\mathcal{L}}$

We prove that the above functor is pro-represtentable by a formal scheme locally formally of finite type. Notice that by remark 6.1.1 one may assume that $b$ satisfies a decency equation for some $s$. 
62CHAPTER 6. THE RAPOPORT-ZINK SPACE FOR LOCAL P-SHTUKAS

Theorem 6.3.1. The functor $\underline{\mathcal{M}}_{\underline{\underline{L}}_{0}}^{\hat{Z}}:\left(\mathcal{N}_{i l p} \llbracket \llbracket \downarrow\right)^{o} \rightarrow$ Sets is pro-representable by a formal scheme over $\operatorname{Spf} k \llbracket \zeta \rrbracket$ which is locally formally of finite type. Its underlying reduced subscheme equals $X_{Z}(b)$.

Recall that a formal scheme over $k \llbracket \zeta \rrbracket$ in the sense of [EGA, $\left.\mathrm{I}_{\text {new }}, 10\right]$ is called locally formally of finite type if it is locally noetherian and adic and its reduced subscheme is locally of finite type over $k$. It is called formally of finite type if in addition it is quasi-compact.

Proof. Consider the universal local $\mathbb{P}$-shtuka $\underline{\mathcal{L}}_{\text {univ }}$ over $\widehat{\mathcal{F}} \ell_{\mathbb{P}}$ (see proposition 4.1.8). Let $\mathcal{M}_{\underline{\underline{L}}_{0}}^{\hat{z}}$ be the closed ind-subscheme of $\widehat{\mathcal{F}} \ell_{\mathbb{P}}$ over which $\underline{\mathcal{L}}_{\text {univ }}$ is bounded by $\hat{Z}$. By construction $\mathcal{M}_{\underline{\underline{L}}_{0}}^{\hat{Z}}$ pro-represents the functor $\underline{\mathcal{M}}_{\underline{\underline{L}}_{0}}^{\hat{Z}}$. It is clear that the reduced ind-subscheme equals $X_{Z}(b)$.

By rigidity of quasi-isogeny the functor $\underline{\mathcal{M}}_{\underline{\underline{L}}_{0}}^{\hat{Z}}$ is equivalent to the following functor

$$
\begin{aligned}
& \left(\mathcal{N} i l p_{k \llbracket \llbracket \rrbracket}\right)^{o} \rightarrow \mathcal{S e t s} \\
& S \longmapsto\left\{(\underline{\mathcal{L}}, \bar{\delta}) ; \underline{\mathcal{L}} \in O b\left(\underline{\mathcal{M}}_{\underline{\mathbb{L}}_{0}}(S)\right) \text { is bounded by } \hat{Z}\right. \text { and } \\
& \left.\delta: \underline{\mathcal{L}} \rightarrow \underline{\mathbb{L}}_{0 S} \text { is a quasi-isogeny }\right\} / \sim .
\end{aligned}
$$

We may take a representation $\iota: \mathbb{P} \rightarrow G L_{N, \mathbb{D}}$, for some integer $N$, which factors through $H:=S L_{N, \mathbb{D}}$ with quasi-affine quotient $H / \mathbb{P}$, see proposition 4.1.3. The representation $\iota$ induces the following 1-morphism

$$
\mathscr{H}^{1}(\iota): \mathscr{H}^{1}(S, L \mathbb{P}) \rightarrow \mathscr{H}^{1}(S, L H) .
$$

Now let $\mathcal{M}^{n}$ be the closed ind-subschem of $\underline{\mathcal{M}}_{\underline{\mathbb{L}}_{0}}^{\hat{\hat{Z}}}$ defined by the following sub functor of $\underline{\mathcal{M}}_{\underline{\underline{L}}_{0}}^{\hat{Z}}$

$$
\begin{aligned}
& \underline{\mathcal{M}}^{n}:\left(\mathcal{N} i l p_{k \llbracket \zeta \rrbracket}\right)^{o} \rightarrow \text { Sets } \\
& S \longmapsto\left\{(\underline{\mathcal{L}}, \bar{\delta}) ;(\underline{\mathcal{L}}, \bar{\delta}) \text { is in } \underline{\mathcal{M}}_{\underline{\underline{L}}_{0}}^{\hat{\mathrm{Z}}}(S)\right. \text { and } \\
& \left.\mathscr{H}^{1}(\iota)(\delta) \text { is bounded by } 2 n \rho^{\vee}\right\} / \sim \text {. }
\end{aligned}
$$

Where $\rho^{\vee}$ is the halfsum of all positive coroots of $H$. Note that here the boundedness condition is the one defined in definition 6.2.1. 
Lemma 6.3.2. The ind-scheme $\mathcal{M}^{n}$ representing the above functor is a $\zeta$-adic noetherian formal closed subscheme of $\mathcal{M}$ over $k \llbracket \zeta \rrbracket$.

Proof. Since $H / \mathbb{P}$ is quasi-affine, the induced morphism $f: \widehat{\mathcal{F}}_{\mathbb{P}} \rightarrow \widehat{\mathcal{F}}_{H}$ is a locally closed embedding by proposition 6.2.7.

The representation $\iota$ induces the following functor

$$
\iota_{*}: \widehat{\mathfrak{S} h} t_{\mathbb{P}} \rightarrow \widehat{\mathfrak{S h}}_{H} \text {. }
$$

Let $\underline{\mathbb{E}}_{0}:=\iota_{*} \underline{\mathbb{L}}_{0}$ and view $\widehat{\mathcal{F} \ell_{H}}$ as a moduli space representing the functor $\underline{\mathcal{M}}_{\mathbb{H}_{0}}$, parametrizing $H$-shtukas together with an isogeny $\delta_{H}$ to $\underline{\mathbb{H}}_{0}$, see 4.1 .8 . Let $\widehat{\mathcal{F}}_{\mathbb{P}}^{\preceq n}$ be the closed ind-subscheme of $\widehat{\mathcal{F}}_{\mathbb{P}}$ defined by the Cartesian diagram

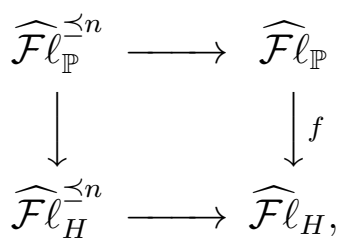

here $\widehat{\mathcal{F}}_{\mathbb{P}}^{\preceq n}$ is defined by bounding $\delta_{H}$ by $2 n \rho^{\vee}$. Note that $\widehat{\mathcal{F}} \ell_{\mathbb{P}}^{\preceq n}$ is a $\zeta$ adic noetherian formal scheme over $k \llbracket \zeta \rrbracket$. Indeed since $f$ is a locally closed embedding thus

$$
\widehat{\mathcal{F}}_{\mathbb{P}}^{\preceq n} \times_{\operatorname{Spf} k \llbracket \zeta \rrbracket} k \llbracket \zeta \rrbracket /\left\langle\zeta^{i}\right\rangle=\widehat{\mathcal{F}}_{\mathbb{P}} \times_{\widehat{\mathcal{F}} \widehat{H}_{H}} \widehat{\mathcal{F}}_{H}^{\preceq n} \times_{\operatorname{Spf} k \llbracket \zeta \rrbracket} \mathrm{V}\left(\zeta^{i}\right)
$$

is a scheme locally of finite type with underlying topological space $f^{-1}\left(\mathcal{F} \ell_{H}^{\preceq n}\right)$, then our claim follows from [EGA, $\mathrm{I}_{\text {new }}$, Cor 10.6.4].

Consider the closed immersion

$$
\mathcal{M}^{n}(i):=\mathcal{M}^{n} \times_{\widehat{\mathcal{F}} \ell_{\mathbb{P}}}^{\preceq n} \mathrm{~V}\left(\zeta^{i}\right) \hookrightarrow \widehat{\mathcal{F}}_{\mathbb{P}}^{\preceq n} \times_{\operatorname{Spf} k \llbracket \zeta \rrbracket} \operatorname{Spec} k \llbracket \zeta \rrbracket /\left(\zeta^{i}\right) .
$$

Clearly we have $\mathcal{M}^{n}(i)(k)=\mathcal{M}^{n}(k)$. We may thus argue, as above, that $\mathcal{M}^{n}=\lim _{\longrightarrow} \mathcal{M}^{n}(i)$ is a $\zeta$-adic noetherian formal scheme .

Now for each number $n \in \mathbb{N}$ we define the following sub functor of $\underline{\mathcal{M}}_{\underline{\mathbb{L}}_{0}}^{\hat{Z}}$

$$
\begin{aligned}
& \underline{\mathcal{M}}_{n}:\left(\mathcal{N} i l p_{k \llbracket \zeta \rrbracket}\right)^{o} \rightarrow \text { Sets } \\
& S \longmapsto\left\{(\underline{\mathcal{L}}, \bar{\delta}) ;(\underline{\mathcal{L}}, \bar{\delta}) \text { is in } \underline{\mathcal{M}}_{\underline{\mathbb{L}}_{0}}^{\hat{Z}}(S)\right. \\
& \text { and for any closed point } \\
& \left.s \text { in } S, \mathscr{H}(\iota)\left(\delta_{s}\right) \text { is bounded by } 2 n \rho^{\vee}\right\} / \sim \text {. }
\end{aligned}
$$


64CHAPTER 6. THE RAPOPORT-ZINK SPACE FOR LOCAL P-SHTUKAS

This functor is represented by an ind-scheme $\mathcal{M}_{n}$ which is the formal completion of $\underline{\mathcal{M}}_{\mathbb{L}_{0}}^{\hat{Z}}$ along $\left(\mathcal{M}^{n}\right)_{\text {red }}$.

Claim: $\mathcal{M}_{n}$ is formally of finite type over $\operatorname{Spf} k \llbracket \zeta \rrbracket$.

We break the proof of this claim into several lemmas, however some may look interesting by their own. We need the following definition

Definition 6.3.3. Let $R$ be a linearly topologized $\mathbb{F}_{q} \llbracket \zeta \rrbracket$-algebra $\lim R_{\alpha}$ for a projective system $\left(R_{\alpha}, u_{\alpha \beta}\right)$ of discrete rings indexed by $\mathbb{N}_{0}$. Suppose that all maps $R \rightarrow R_{\alpha}$ are surjective, and the kernels $I_{\alpha}:=\operatorname{ker} u_{\alpha, 0} \subset R_{\alpha}$ are nilpotent. A local $\mathbb{P}$-shtuka over $\operatorname{Spf} R$ is a projective system $\left(\underline{\mathcal{L}}_{\alpha}\right)_{\alpha \in \mathbb{N}_{0}}$ of local $\mathbb{P}$-shtukas $\underline{\mathcal{L}}_{\alpha}$ over $R_{\alpha}$ with $\underline{\mathcal{L}}_{\alpha-1} \cong \underline{\mathcal{L}}_{\alpha} \otimes_{R_{\alpha}} R_{\alpha-1}$.

Lemma 6.3.4. Let $R$ in $\mathcal{N} i l p_{k \llbracket \zeta \rrbracket}$ be as in the above definition. The pull back functor defines a bijection between the category of local $\mathbb{P}$-shtukas over Spec $R$ bounded by $\hat{Z}$ and the category of formal $\mathbb{P}$-shtukas over $\operatorname{Spf} R$ bounded by $\hat{Z}$.

Proof. Since $R$ is in $\mathcal{N} i l p_{k \llbracket \zeta \rrbracket}$ there is an integer $e \in \mathbb{N}$ such that $\zeta^{e}=0$ on $R$. Let $\hat{\mathcal{L}}:=\left(\hat{\mathcal{L}}_{n}\right)_{n \in \mathbb{N}_{0}}$ be a local $\mathbb{P}$-shtuka over $\operatorname{Spf} R$. There is an étale cover $R_{0}^{\prime} \rightarrow R_{0}$ which trivializes $\hat{\mathcal{L}}_{0}$ and hence a unique étale $R$-algebra $R^{\prime}$ with $R^{\prime} \otimes_{R} R_{0} \cong R_{0}^{\prime}$ by [SGA, Théorème I.8.3]. This gives rise to the trivializations $\hat{\mathcal{L}}_{n} \otimes R_{n}^{\prime} \cong\left(L^{+} \mathbb{P}_{R_{n}^{\prime}}, b_{n} \hat{\sigma}^{*}\right)$ over $R_{n}^{\prime}:=R^{\prime} \otimes_{R} R_{n}$. Here $b_{n} \in L P_{\eta}\left(R_{n}^{\prime}\right)=P_{\eta}\left(R_{n}^{\prime} \llbracket z \rrbracket\left[\frac{1}{z-\zeta}\right]\right)$ and $b_{n} \otimes_{R_{n}^{\prime}} R_{n-1}^{\prime}=b_{n-1}$.

Take a faithful representation $\mathbb{P} \hookrightarrow H$ as before. This induces a locally closed immersion $\iota: \widehat{\mathcal{F} \ell_{\mathbb{P}}} \rightarrow \widehat{\mathcal{F} \ell_{H}}$ and also an ind-scheme structure on $\widehat{\mathcal{F}}_{\mathbb{P}}:=\lim _{\vec{n}} \widehat{\mathcal{F}}_{\mathbb{P}}^{(n)}$. Since $Z_{e}:=\hat{Z} \times_{\operatorname{Spf} k \llbracket \zeta \rrbracket} \operatorname{Spec} k \llbracket \zeta \rrbracket /\left(\zeta^{e}\right)$ has the same underlying topological space than $Z$, it is quasi-compact. Hence there is $N \in \mathbb{N}$ such that $Z_{e} \subseteq \widehat{\mathcal{F}} \widehat{P}_{\mathbb{P}}^{(N)}$. Thus for any $n \in \mathbb{N}$, the morphism $b_{n}:$ Spec $R_{n}^{\prime} \rightarrow \widehat{\mathcal{F} \ell_{\mathbb{P}}}$ factors through $\widehat{\mathcal{F}}_{\mathbb{P}}^{(N)}$, i.e. we have the diagram

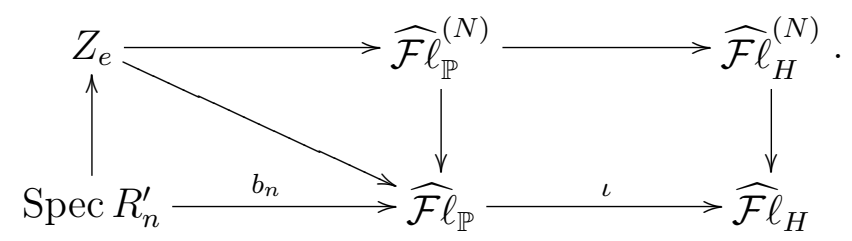

Thus we obtain $b_{\infty}^{\prime}:=\lim _{n \rightarrow \infty} b_{n} \in L P_{\eta}^{(N)}\left(R^{\prime}\right)$, where $L P_{\eta}^{(N)}=L P_{\eta} \times_{\widehat{\mathcal{F}} \ell_{\mathbb{P}}}$ $\widehat{\mathcal{F}}_{\mathbb{P}}^{(N)}$. This gives the local $\mathbb{P}$-shtuka $\left(L \mathbb{P}, b_{\infty}^{\prime} \hat{\sigma}^{*}\right)$ over Spec $R^{\prime}$ which carries descent data from the $\underline{\hat{\mathcal{L}}}_{n}$ and hence induces a local $\mathbb{P}$-shtuka over Spec $R$. 
Let us come back to the proof of the claim. For each $m \geq n$ let $\mathcal{M}_{n}^{m}$ be the formal completion of $\mathcal{M}^{m}$ along $\left(\mathcal{M}_{n}\right)_{\text {red }}$. It is an adic formal scheme over $k \llbracket \zeta \rrbracket$. Let $U$ be an affine open subscheme of $\left(\mathcal{M}_{n}\right)_{\text {red. }}$ This defines an affine open formal subscheme $\operatorname{Spf} R_{m}$ of $\mathcal{M}_{n}^{m}$ with underlying set $U$. Let $R$ be the inverse limit of the projective system $R_{m+1} \rightarrow R_{m}$ and let $\mathfrak{a}_{m}$ denote the ideal such that $R_{m}=R / \mathfrak{a}_{m}$. Let $J$ be the inverse image in $R$ of the largest ideal of definition in $R_{n}$. We want to show that $R$ is $J$-adic.

As we will see below, the rigidity of quasi isogenies (proposition 3.1.3) together with lemma 6.3.4 and proposition 6.2.5 imply the above claim.

Lemma 6.3.5. For any integer $c>0$ there is an integer $m_{0}$ such that for any $m \geq m_{0}$ there exist an oblique arrow which fits into the following commutative diagram

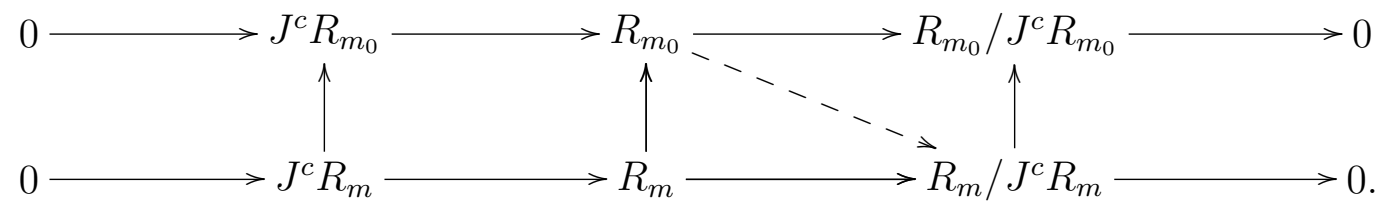

Proof. Let $\underline{\mathcal{L}}_{m}$ be the universal local $\mathbb{P}$-shtuka over Spf $R_{m}$. Consider the local $\mathbb{P}$-shtuka $\underline{\mathcal{L}}=\lim _{\underline{\mathcal{L}}}$ over $\operatorname{Spf} R$. This lifts to a local $\mathbb{P}$-shtuka $\underline{\mathcal{L}}$ over Spec $R$ by lemma 6.3.4. Consider the closed immersion $i$ : Spec $R /$ $J^{c} \rightarrow$ Spec $R$ and the pull-back local $\mathbb{P}$-shtuka $i^{*} \mathcal{L}$. By rigidity of quasiisogenies the quasi-isogeny $\delta_{n}$ over $R / J=R_{n} / J$ lifts to a quasi-isogeny $\delta$ over $R / J^{c}$. In addition the isogeny $\mathscr{H}^{1}(\iota)(\delta)$ is bounded by $2 m_{0} \rho^{\vee}$ for some $m_{0}$, by proposition 6.2 .5 . Now the tuple $\left(i^{*} \underline{\mathcal{L}}, \delta\right)$ induces the desired morphism by the universal property of $\mathcal{M}_{n}^{m_{0}}$.

By the above lemma we see that for all $m \geq m_{0}$ the morphism

$$
R_{m} / J^{c} R_{m} \rightarrow R_{m_{0}} / J^{c} R_{m_{0}}
$$

is an isomorphism. Thus the chain

$$
\mathfrak{a}_{1}+J^{c} \supseteq \mathfrak{a}_{2}+J^{c} \supseteq \cdots \supseteq \mathfrak{a}_{i}+J^{c} \supseteq \ldots
$$

stabilizes. Now consider the chain

$$
\mathcal{J}_{1} \supseteq \mathcal{J}_{2} \supseteq \ldots \supseteq \mathcal{J}_{i} \ldots
$$


66CHAPTER 6. THE RAPOPORT-ZINK SPACE FOR LOCAL P-SHTUKAS

where $\mathcal{J}_{c}$ is the intersection of the ideals in the chain (6.3.4) and therefore equals $\mathfrak{a}_{m}+J^{c}$ for $m \gg 0$. Note that $\mathcal{J}_{1}=J$ and $\mathcal{J}_{m+1}+\mathcal{J}_{1}^{m}=\mathcal{J}_{m}$. Since $\mathcal{J}_{1} / \mathcal{J}_{2}$ is a finite type $R$-module the claim follows from [EGA, $O_{I}$ 7.2.2].

For two closed points $\underline{x}_{1}:=\left(\underline{\mathcal{L}}_{1}, \delta_{1}\right)$ and $\underline{x}_{2}:=\left(\underline{\mathcal{L}}_{2}, \delta_{2}\right)$ define

$$
\tilde{d}\left(\underline{x}_{1}, \underline{x}_{2}\right):=\min \left\{n \in \mathbb{N}_{0} ; \mathscr{H}^{1}(\iota)\left(\delta_{1}\right)^{-1} \mathscr{H}^{1}(\iota)\left(\delta_{2}\right) \leq 2 n \rho^{\vee}\right\} .
$$

Lemma 6.3.6. There is an integer $d_{0} \in \mathbb{N}_{0}$ such that

$$
\max \left\{\tilde{d}\left(x, \underline{\mathcal{M}}_{\underline{\mathbb{L}}_{0}}^{\hat{z}}\left(\mathbb{F}_{q^{s}}\right)\right) ; x \in \underline{\mathcal{M}}_{\underline{\mathbb{L}}_{0}}^{\hat{z}}(\bar{k})\right\} \leq d_{0} .
$$

Proof. Let $\underline{x}:=\left(\underline{\mathcal{L}}^{+}, \delta\right)$ be a closed point of $\underline{\mathcal{M}}_{\underline{L}_{0}}^{\hat{z}}$. Take a trivialization $\left(\underline{\mathcal{L}}^{+}, \delta\right) \cong\left(\left(L^{+} G, h \sigma^{*}\right), g\right)$ for the pair associated to the point $\underline{x}$. Since the local $\mathbb{P}$-shtuka $\left(L^{+} G, h \sigma^{*}\right)$ is bounded by $\hat{Z}$ and by the definition of the boundedness condition (see definition 6.2.6) $Z$ is quasi-compact, we have $d\left(g, \bar{b} \hat{\sigma}^{*} g\right)<c$, for some natural number $c$. Since $\bar{b}$ satisfies the decency condition, therefore according to theorem 6.1.3 there is an integer $d_{0}$ which is independent of the chosen point $\underline{x}$, and $g^{\prime} \in L G\left(\mathbb{F}_{q^{s}}\right)$, such that the distance of $g$ and $g^{\prime}$ in the Bruhat-Tits building of $G:=P_{\eta}$ is less than $d_{0}$. Now we consider the associated point $\left(\left(L^{+} \mathbb{P}, g^{\prime-1} \bar{b} \sigma^{*} g^{\prime}\right), g^{\prime}\right)$ of $\underline{\mathcal{M}}_{\mathbb{L}_{0}}^{\hat{Z}}\left(\mathbb{F}_{q^{s}}\right)$. Notice that by functorial properties of Bruhat-Tits buildings (see [La1]), $\iota$ induces an injective isometric map of Bruhat-Tits buildings $\mathscr{B}\left(P_{\eta}\right) \rightarrow \mathscr{B}(H)$, and hence the lemma follows.

Set $B(y)=B\left(y, d_{0}\right):=\left\{x \in \mathcal{M}_{\underline{\underline{\underline{Z}}}_{0}}^{\hat{\hat{Z}}}(k) ; \tilde{d}(x, y) \leq d_{0}\right\}$ and $B_{n}(y)=$ $B\left(y, d_{0}\right) \cap \mathcal{M}_{n}(k)$. Note that these are closed subsets.

For each integer $r$ let

$$
\mathcal{Z}_{n}^{r}=\bigcup_{\left.y \in \mathcal{M}\left(\mathbb{F}_{q^{s}}\right), \tilde{d}\left(\underline{\mathbb{L}}_{0}, i d\right), y\right) \geq r} B_{n}(y)
$$

If $y \notin \mathcal{M}_{n+d_{0}}(k)$, i.e. $\tilde{d}\left(\left(\underline{\mathbb{L}}_{0}, i d\right), y\right)>n+d_{0}$ and $x \in \mathcal{M}_{n}(k)$ then

$$
\tilde{d}(x, y) \geq\left[\tilde{d}\left(\left(\underline{\mathbb{L}}_{0}, i d\right), x\right)-n\right]+\tilde{d}(x, y) \geq \tilde{d}\left(\left(\underline{\mathbb{L}}_{0}, i d\right), y\right)-n>d_{0}
$$

and thus $B_{n}(y)=\emptyset$. We get

$$
\mathcal{Z}_{n}^{r}=\bigcup_{y \in \mathcal{M}_{n+d_{0}}\left(\mathbb{F}_{q^{s}}\right), \tilde{d}\left(\left(\mathbb{L}_{0}, i d\right), y\right) \geq r} B_{n}(y) .
$$


Since $\left(\mathcal{M}_{n+d_{0}}\right)_{\text {red }}=\left(\mathcal{M}^{n+d_{0}}\right)_{\text {red }}$ and $\left(\mathcal{M}^{n+d_{0}}\right)_{\text {red }}$ is of finite type (see proof of lemma 6.3.2) hence this union is finite.

Let $\mathcal{U}_{n}^{r}$ be the open formal sub-scheme of $\mathcal{M}_{n}$ whose underlying reduced set is $\mathcal{M}_{n} \backslash \mathcal{Z}_{n}^{r}$. We claim that the following chain of formal sub-schemes of $\mathcal{M}_{n}$

$$
\mathcal{U}_{n}^{r} \hookrightarrow \mathcal{U}_{n+1}^{r} \cdots \subset \underline{\mathcal{M}}_{\underline{\mathbb{L}}_{0}}^{\hat{z}}
$$

stabilizes. By the definition of $\mathcal{M}_{n}$ it is enough to verify this on the underlying set of points. Suppose that there is some element $x \in \mathcal{U}_{n+1}^{r}(k) \backslash \mathcal{M}_{n}(k)$. By lemma 6.3.6 there exist a $y \in \underline{\mathcal{M}}_{\underline{\underline{L}}_{0}}^{\hat{Z}}\left(\mathbb{F}_{q^{s}}\right)$ such that $\tilde{d}(x, y) \leq d_{0}$. Then

$$
\tilde{d}\left(\left(\underline{\mathbb{L}}_{0}, i d\right), x\right) \leq \tilde{d}\left(\left(\underline{\mathbb{L}}_{0}, i d\right), y\right)+\tilde{d}(x, y)<r+d_{0},
$$

therefore if $n \geq r+d_{0}$ then $\tilde{d}\left(\left(\underline{\underline{L}}_{0}, i d\right), x\right) \leq n$ which is a contradiction and consequently there is no such $x$.

Let $\mathcal{U}^{r}=\bigcup_{n} \mathcal{U}_{n}^{r}$ (which equals $\mathcal{U}_{n}^{r}$ for $n \geq r+d_{0}$ ). Note that every geometric point of $\mathcal{M}_{\underline{L}_{0}}^{\hat{Z}}$ lies in the union of $\mathcal{U}^{r}$ s. Now consider the chain

$$
\mathcal{U}^{r} \hookrightarrow \mathcal{U}^{r+1} \cdots \hookrightarrow \underline{\mathcal{M}}_{\underline{\underline{L}}_{0}}^{\hat{Z}}
$$

of open immersions of formal schemes formally of finite type, note that $\mathcal{U}^{r}$ is open in $\mathcal{M}_{\underline{\underline{L}}_{0}}^{\hat{z}}$. Indeed the underlying topological space of $\mathcal{U}^{r}$ is open in $\mathcal{M}_{n}$ for every $n$ and the ind-scheme $\mathcal{M}_{\mathbb{L}_{0}}^{\hat{z}}$ carries the limit topology of the limit over the $\mathcal{M}_{n}$. This shows that the formal scheme $\mathcal{U}^{r}$ equals the formal completion of the open ind-scheme $\underline{\mathcal{M}}_{\mathbb{\mathbb { L }}_{0}}^{\hat{Z}}\left|\mathcal{U}^{r}\right|$ of $\underline{\mathcal{M}}_{\underline{\underline{U}}_{0}}^{\hat{\mathrm{Z}}}$ supported on $\left|\mathcal{U}^{r}\right|$ along the whole set $\left|\mathcal{U}^{r}\right|$ and thus $\underline{\mathcal{M}}_{\underline{\underline{L}}_{0}}^{\hat{Z}}\left|\mathcal{U}^{r}\right|=\mathcal{U}^{r}$. Since $\mathcal{U}^{r}$ is locally of finite type so is $\left.\mathcal{M}_{\mathbb{L}_{0}}^{\hat{Z}}\right|_{\left|\mathcal{U}^{r}\right|}$. This implies that $\mathcal{M}=\bigcup_{r} \mathcal{U}^{r}$ is locally formally of finite type as well. 
68CHAPTER 6. THE RAPOPORT-ZINK SPACE FOR LOCAL P-SHTUKAS 


\section{Chapter 7}

\section{The Uniformization Theorem}

\subsection{The Uniformization Theorem}

For a global $\mathfrak{G}$-shtuka $\underline{\mathcal{G}}_{0}$ over $k$ we let $I(Q)=I_{\underline{\mathcal{G}}_{0}}(Q)$ denote the group $Q I \operatorname{sog}_{k}\left(\underline{\mathcal{G}}_{0}\right)$ of quasi-isogenies of $\underline{\mathcal{G}}_{0}$. Let $\left(\underline{\mathcal{L}}_{\nu}\right)_{\nu}$ denote the associated tuple of local $\mathbb{P}_{\nu}$-shtukas under the global-local functor $\widehat{\Gamma}$, see section 3.2. Let $J_{\mathcal{L}_{\nu}}\left(Q_{\nu}\right)$ denote the group $Q I \operatorname{sog}_{k}\left(\underline{\mathcal{L}}_{\nu}\right)$ of quasi-isogenies of $\underline{\mathcal{L}}_{\nu}$. Recall that the group of quasi-isogenies of a local $\mathbb{P}$-shtuka $\underline{\mathcal{L}}$ acts naturally on the ind-scheme $\widehat{\mathcal{F}} \ell_{\mathbb{P}_{\nu}}$ and also on the Rapoport-Zink space for $\mathbb{P}$-shtukas $\mathcal{M}_{\mathcal{L}_{\nu}}^{\hat{Z}}$, see proposition 4.1.8 and theorem 6.3.1. This in particular illustrates that $J_{\underline{\mathcal{L}}_{\nu}}\left(Q_{\nu}\right)$ may appear as a sort of symmetries of the associated affine DeligneLusztig variety.

Especially we see that the group $I(Q)$ acts on the product $\prod_{\nu} \mathcal{M}_{\underline{\underline{Z}} \nu}^{\widehat{Z}_{\nu}}$ of the associated Rapoport-Zink spaces (resp. $\prod_{\nu} \widehat{\mathcal{F}}_{\mathbb{P}_{\nu}}$ ) via the natural morphism

$$
I(Q) \rightarrow \prod_{\nu} J_{\underline{\mathcal{L}}_{\nu}}\left(Q_{\nu}\right)
$$

Let $\mathcal{S}$ be an finite subset of the characteristic places $\nu$ of $\underline{\mathcal{G}}_{0}$ and $\Gamma \subseteq$ $\prod_{\nu \in \mathcal{S}} J_{\underline{\mathcal{L}}_{\nu}}\left(Q_{\nu}\right)$ be a discrete subgroup. We say that $\Gamma$ is separated if it is separated in the profinite topology, i.e. for every $g \in \Gamma$ there is a normal subgroup of finite index that does not contain $g$.

Proposition 7.1.1. Let $\Gamma \subseteq \prod_{\nu} J_{\underline{\mathcal{L}}_{\nu}}\left(Q_{\nu}\right)$ be a separated discrete subgroup. Then the quotient $\Gamma \backslash \prod_{\nu} \mathcal{M}_{\mathcal{L}}^{\widehat{Z}}$ is a locally noetherian, adic formal algebraic $\operatorname{Spf} k \llbracket \zeta \rrbracket$-stack locally formally of finite type over $\operatorname{Spf} k$. Moreover, the 1morphism $\prod_{\nu} \mathcal{M}_{\underline{\mathcal{L}}_{\nu}}^{\widehat{Z}} \rightarrow \Gamma \backslash \prod_{\nu} \mathcal{M}_{\underline{\mathcal{L}}_{\nu}}^{\widehat{Z}}$ is adic and (formally) étale.

Before proving the above proposition let us state the following lemma. 
Lemma 7.1.2. Let $\Gamma_{\nu} \subseteq J_{\underline{\mathcal{L}}_{\nu}}\left(Q_{\nu}\right)$ be a separated discrete subgroup. Consider $\widehat{\mathcal{F}} \ell_{\mathbb{P}_{\nu}}$ as a moduli space for $\underline{\mathcal{M}}_{\underline{\mathcal{L}}_{\nu}}$, see proposition 4.1.8. Let $U_{\nu} \subset \mathcal{F} \ell_{\mathbb{P}_{\nu}}$ be a quasi-compact subscheme. Then the set

$$
\left\{\gamma \in \Gamma ; \gamma U_{\nu} \cap U_{\nu} \neq \emptyset\right\}
$$

is finite.

Proof. Any point $\underline{x} \in U_{\nu}(\bar{k})$ can be represented by a tuple $(\underline{\mathbb{L}}, g)$, where $\underline{\mathbb{L}}:=\left(L^{+} \mathbb{P}_{\nu}, b \sigma^{*}\right)$ is a trivialized local $\mathbb{P}_{\nu^{-}}$shtuka over $\bar{k}$ and $g \in L G(\bar{k})$. By proposition 4.1 .5 the preimage $\widetilde{U} \subseteq L \mathbb{P}_{\nu}$ of $U_{\nu}$ under the projection $L \mathbb{P}_{\nu} \rightarrow \mathcal{F} \ell_{\mathbb{P}_{\nu}}$ is quasi-compact. Consider the morphism

$$
\begin{aligned}
\widetilde{U}(\bar{k}) \times \widetilde{U}(\bar{k}) & \rightarrow \mathcal{F} \ell_{\mathbb{P}_{\nu}}(\bar{k}) \\
g \times g^{\prime} & \mapsto g^{\prime} g^{-1} \cdot L^{+} \mathbb{P}_{\nu} / L^{+} \mathbb{P}_{\nu}
\end{aligned}
$$

Since $\mathcal{F} \ell_{\mathbb{P}_{\nu}}(\bar{k})$ is an ind-scheme, this morphism factors through some $V \subset$ $\mathcal{F} \ell_{\mathbb{P}_{\nu}}$ of finite type. We may assume that $b$ is decent, so the group of quasiisogenies $J_{\mathcal{L}_{\nu}}\left(Q_{\nu}\right) \subset L G(\bar{k})$ is defined over some finite extension $L / \mathbb{F}_{q}$, see remark 6.1.2. Let $\gamma \in \Gamma$, if $\underline{x} \in U_{\nu}$ and $\gamma \underline{x} \in U_{\nu}$ then the image of $\gamma$ under the projection map $\pi: L G(\bar{k}) \rightarrow \mathcal{F} \ell_{\mathbb{P}_{\nu}}$ maps to $V(L)$. Thus $\gamma$ lies in $S=\pi^{-1}(V(L)) \cap J\left(Q_{\nu}\right)$. On the other hand $\Gamma_{\nu}$ is discrete and thus has finite intersection with the compact set $S$.

Remark 7.1.3. One can state a variant of the above lemma for a separated discrete subgroup $\Gamma$ of $\prod_{\nu} J_{\mathcal{L}_{\nu}}\left(Q_{\nu}\right)$. Namely, let $U_{\nu}$ be as above and set $U=\prod_{\nu} U_{\nu}$, then one can show, in a similar way as in the above proof, that

$$
\{\gamma \in \Gamma ; \gamma U \cap U \neq \emptyset\}
$$

is finite.

Proof. of proposition 7.1.1 By theorem 6.3.6 we may choose a constant $d_{\nu}$ such that any ball in $\mathcal{M}_{\underline{\mathcal{L}}_{\nu}}^{\widehat{Z}}(\bar{k})$ with radius $d_{\nu}$ contains a rational point in $\mathcal{M}_{\underline{\mathcal{L}}_{\nu}}^{\widehat{Z}}\left(L_{\nu}\right)$ for the finite extension $L_{\nu} / \mathbb{F}_{q}$ (see proof of the above lemma). Let $d$ be the maximum of the integers $d_{\nu}$ and $L$ be the compositum of the fields $L_{\nu}$. Let $x:=\left(x_{\nu}\right)$ be a closed point of $\prod_{\nu} \mathcal{M}_{\underline{\mathcal{L}}_{\nu}}^{\widehat{Z}}$. Note that since the underlying reduced subscheme of $\mathcal{M}_{\underline{\mathcal{L}}_{\nu}}^{\widehat{Z}}$ is of finite type and therefore has

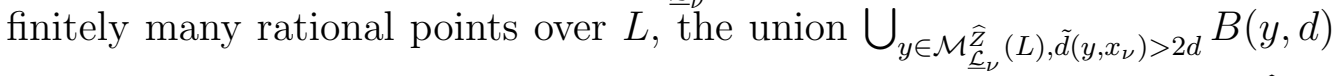
defines a closed subscheme $\mathcal{Z}_{\nu}$. Define the open neighborhood $U_{\nu}=\mathcal{M}_{\underline{\mathcal{L}}_{\nu}}^{\widehat{Z}} \backslash$ $\mathcal{Z}_{\nu}$ of $x_{\nu}$. Set $U_{x}:=\prod_{\nu} U_{\nu}$. One may easily see that $\gamma \cdot U_{x}=U_{\gamma \cdot x}$ and that 
the open neighborhoods $U_{x}$ are bounded and cover $\prod_{\nu} \mathcal{M}_{\underline{\mathcal{L}}_{\nu}}^{\widehat{\mathrm{Z}}}$, for varying $x \in \prod_{\nu} \mathcal{M}_{\underline{\mathcal{L}}_{\nu}}^{\widehat{Z}}(L)$. Let $I \subset \prod_{\nu} \mathcal{M}_{\underline{\mathcal{L}}_{\nu}}^{\widehat{Z}}$ be a set of representatives of the $\Gamma$ orbits in $\prod_{\nu} \mathcal{M}_{\underline{\mathcal{L}}_{\nu}}^{\widehat{Z}}(L)$.

For a fixed $i \in \bar{I}$, since $\Gamma$ is separated by the remark 7.1 .3 we may choose a normal subgroup $\Gamma^{\prime} \subset \Gamma$ of finite index in $\Gamma$ such that $U_{i} \cap \gamma^{\prime} U_{i}=\emptyset$ for all $\gamma^{\prime} \neq 1$ in $\Gamma^{\prime}$. Note that it is enough to show that $\Gamma^{\prime} \backslash \prod_{\nu} \mathcal{M}_{\underline{\mathcal{L}}_{\nu}}^{\widehat{Z}}$ is Deligne-Mumford and the projection

$$
\prod_{\nu} \mathcal{M}_{\underline{\mathcal{L}}_{\nu}}^{\widehat{Z}} \rightarrow \Gamma^{\prime} \backslash \prod_{\nu} \mathcal{M}_{\underline{\mathcal{L}}_{\nu}}^{\widehat{Z}}
$$

is adic, see remark 2.1.6. Let $V_{i}$ denote the union of the image in $\Gamma^{\prime} \backslash \prod_{\nu} \mathcal{M}_{\underline{\mathcal{L}}_{\nu}}^{\widehat{Z}}$ of all translations $\gamma^{\prime} U_{i}$, for $\gamma^{\prime} \in \Gamma^{\prime}$. Then the composition of the open immersion

$$
\bigsqcup_{i \in I} V_{i} \rightarrow \Gamma^{\prime} \backslash \prod_{\nu} \mathcal{M}_{\underline{\mathcal{L}}_{\nu}}^{\widehat{Z}}
$$

followed by the projection $\Gamma^{\prime} \backslash \prod_{\nu} \mathcal{M}_{\underline{\mathcal{L}}_{\nu}}^{\widehat{Z}} \rightarrow \Gamma \backslash \prod_{\nu} \mathcal{M}_{\underline{\mathcal{L}}_{\nu}}^{\widehat{Z}}$ gives the desired étale presentation.

Let $\left(\underline{\mathcal{G}}_{0}, \gamma_{0}\right)$ be a global $\mathfrak{G}$-shtuka, bounded by $\widehat{Z}_{\underline{\underline{\nu}}}:=\left(\widehat{Z}_{\nu}\right)_{\nu}$, with level $H$-structure $\gamma_{0}$, in $\nabla_{n}^{H} \mathscr{H}^{1}(C, \mathfrak{G})^{\underline{\underline{\nu}}}(k)$, where $H \subset \mathfrak{G}\left(\overline{\mathbb{A}} \frac{\nu}{Q}\right)$ is a compact open subgroup supported outside the characteristic sections of $\underline{\mathcal{G}}_{0}$. Let $\left(\underline{\mathcal{L}}_{\nu}\right)_{\nu}$ be the tuple of local shtukas associated with $\underline{\mathcal{G}}_{0}$ via the global-local functor as before. Let $\mathcal{M}_{\underline{\mathcal{L}}_{\nu}}^{\widehat{Z}_{\nu}}$ denote the associated Rapoport-Zink spaces. Let $\left\{T_{i}\right\}$ be a set of representatives of $I(Q)$-orbits of the irreducible components of $\prod_{\nu} \mathcal{M}_{\underline{\mathcal{L}}_{\nu}}^{\widehat{Z}}$.

Theorem 7.1.4. Keep the above notation, we have the following statements

(a) the morphism $\Psi$ induces a $I(Q) \times \mathfrak{G}\left(\mathbb{A} \frac{\nu}{Q}\right)$-equivariant morphism

$$
\Theta^{\prime}: \prod_{\nu} \mathcal{M}_{\underline{\mathcal{L}}_{\nu}}^{\hat{\mathrm{Z}}_{\nu}} \times \mathfrak{G}\left(\mathbb{A}_{\underline{Q}}^{\underline{\nu}}\right) / H \rightarrow \nabla_{n}^{H, \hat{Z}_{\underline{\nu}}} \mathscr{H}^{1}(C, \mathfrak{G})^{\underline{\underline{\nu}}} .
$$

Further more this morphism factors through a $\mathfrak{G}\left(\mathbb{A} \frac{\nu}{Q}\right)$-equivariant morphism

$$
\Theta: I(Q) \backslash \prod_{\nu} \mathcal{M}_{\underline{\mathcal{L}}_{\nu}}^{\hat{\mathcal{Z}}_{\nu}} \times \mathfrak{G}\left(\mathbb{A}_{Q}^{\underline{\nu}}\right) / H \rightarrow \nabla_{n}^{H, \hat{Z}_{\underline{\underline{\nu}}}} \mathscr{H}^{1}(C, \mathfrak{G})^{\underline{\underline{\nu}}} .
$$

of formal algebraic stacks. 
(b) Let $\mathcal{Z}$ denote the union of the images of the $T_{i}$ s under the uniformization morphism. Then $\Theta$ induces the following isomorphism

$$
\Theta_{\mathcal{Z}}: I(Q) \backslash \prod_{\nu} \mathcal{M}_{\underline{\mathcal{L}}_{\nu}}^{\hat{Z}_{\nu}} \times \mathfrak{G}\left(\mathbb{A} \frac{\nu}{Q}\right) / H \rightarrow \nabla_{n}^{H, \hat{Z}_{\underline{\nu}}}, \mathscr{H}^{1}(C, \mathfrak{G})^{\frac{\bar{\nu}}{\mathcal{Z}}}
$$

of formal algebraic stacks.

Notice that in part $(b)$ of the above theorem, the $\Theta\left(T_{i}\right)$ are closed (see theorem 4.1.12), and each $\Theta\left(T_{i}\right)$ intersects finitely many others, thus we may form the completion along their image, see [RZ, paragraph 6.22].

Proof. The map $\Psi$ introduced in theorem 4.1 .12 restricts to the following map

$$
\left.\Psi\right|_{\widehat{Z}}: \prod_{\nu} \mathcal{M}_{\underline{\mathcal{L}}_{\nu}}^{\hat{\mathrm{Z}}_{\nu}} \rightarrow \nabla_{n}^{\hat{Z}_{\underline{\nu}}} \mathscr{H}^{1}(C, \mathfrak{G})^{\underline{\underline{\nu}}}
$$

of formal algebraic stacks.

By the Tannakian formalism, a given element $g \in \mathfrak{G}\left(\mathbb{A} \frac{\nu}{Q}\right)$ defines an automorphism of the neutral tensor functor $\omega^{\circ}$ (defined outside $\underline{\nu}$ ). Now consider an $S$ valued point $\left(\underline{\mathcal{L}}_{\nu}^{\prime}, \varphi_{\nu}\right)_{\nu}$ of $\prod_{\nu} \mathcal{M}_{\underline{\mathcal{L}}_{\nu}}^{\hat{Z}_{\nu}}$ and let $\underline{\mathcal{G}}^{\prime}$ denote its image under $\Psi$. Note that there is a unique quai-isogeny $\varrho: \underline{\mathcal{G}}^{\prime} \rightarrow \underline{\mathcal{G}}_{0}$, see remark 4.1.13. This induces a functor $\mathcal{T}_{\varrho}: \mathcal{T}_{\mathcal{G}^{\prime}} \rightarrow \mathcal{T}_{\underline{\mathcal{G}}}$, see 5.2.1. Now these data suffice to establish the morphism

$$
\Theta^{\prime}: \prod_{\nu} \mathcal{M}_{\underline{\mathcal{L}}_{\nu}}^{\hat{Z}_{\nu}} \times \mathfrak{G}\left(\mathbb{A} \frac{\nu}{Q}\right) / H \rightarrow \nabla_{n}^{H, \hat{Z}_{\underline{\nu}}} \mathscr{H}^{1}(C, \mathfrak{G})^{\underline{\underline{\nu}}}
$$

which sends $\left(\underline{\mathcal{L}}_{\nu}, \varphi_{\nu}\right)_{\nu} \times h H$ to $\left(\underline{\mathcal{G}}^{\prime}, H h^{-1} \gamma_{0} \mathcal{T}_{\varrho}\right)$.

The group $I(Q)$ of quasi-isogenies of $\underline{\mathcal{G}}_{0}$ acts on the automorphism group $A u t^{\otimes}\left(\mathcal{T}_{\mathcal{G}_{0}}\right)$ of the Tate functor, see section 5.2. This induces a morphism

$$
\beta: I(Q) \rightarrow A u t^{\otimes}\left(\omega^{\circ}\right) \cong \mathfrak{G}\left(\mathbb{A} \frac{\nu}{Q}\right)
$$

regarding the level $H$-structure $\gamma_{0}$. Hence we get the following morphism

$$
\left(\psi_{\nu}, \beta\right): I(Q) \rightarrow \prod_{\nu} J_{\underline{\mathcal{L}}_{\nu}}\left(Q_{\nu}\right) \times \mathfrak{G}\left(\mathbb{A} \frac{\nu}{Q}\right)
$$

Let $I^{\prime}(Q)$ denote its image. We claim that this is a discrete subgroup of $\prod_{\nu} J\left(Q_{\nu}\right) \times \mathfrak{G}\left(\mathbb{A} \frac{\nu}{Q}\right)$. To show this we take an open subset $U \subset \prod_{\nu} J\left(Q_{\nu}\right) \times$ 
$\mathfrak{G}(\mathbb{A})$ sufficiently small such that any element in $I^{\prime}(Q) \cap U$ induces isomorphisms of local $\mathbb{P}_{\nu}$-shtukas $\underline{\mathcal{L}}_{\nu}$, for every $\nu$. Thus these elements give automorphisms of the global shtuka $\underline{\mathcal{G}}_{0}:=\left(\mathcal{G}, \tau_{0}\right)$, see remark 4.1.13. Now the finiteness of the set $I^{\prime}(Q) \cap U$ follows from remark 6.2.19.

Hence we observe that

$$
I(Q) \backslash \prod_{\nu} \mathcal{M}_{\underline{\mathcal{L}}_{\nu}}^{\hat{\mathrm{Z}}_{\nu}} \times \mathfrak{G}\left(\mathbb{A} \frac{\nu}{Q}\right) / H \cong \coprod_{\Gamma}\left(\Gamma \backslash \prod_{\nu} \mathcal{M}_{\underline{\underline{L}}_{\nu}}^{\hat{\mathrm{Z}}_{\nu}}\right),
$$

where $\Gamma$ runs through a countable set of subgroups of $\prod_{\nu} J_{\underline{\mathcal{L}}_{\nu}}\left(Q_{\nu}\right)$ of the form

$$
\left(\prod_{\nu} J_{\underline{\mathcal{L}}_{\nu}}\left(Q_{\nu}\right) \times g H g^{-1}\right) \cap I(Q) \subseteq \prod_{\nu} J_{\underline{\mathcal{L}}_{\nu}}\left(Q_{\nu}\right) .
$$

One can easily check that these groups $\Gamma$ are separated. Hence $I(Q) \backslash \prod_{\nu} \mathcal{M}_{\underline{\mathcal{L}}_{\nu}}^{\hat{Z}_{\nu}} \times$ $\mathfrak{G}\left(\mathbb{A}_{Q} \underline{\nu}\right) / H$ is a formal algebraic stack by proposition 7.1.1. According to this, the morphism $\Theta^{\prime}$ factors through the following morphism

$$
\Theta: I(Q) \backslash \prod_{\nu} \mathcal{M}_{\underline{\mathcal{Z}}_{\nu}}^{\hat{\mathcal{Z}}_{\nu}} \times \mathfrak{G}\left(\mathbb{A} \frac{\underline{\nu}}{Q}\right) / H \rightarrow \nabla_{n}^{H, \hat{Z}_{\underline{\nu}}} \mathscr{H}^{1}(C, \mathfrak{G})^{\underline{\underline{\nu}}} .
$$

of formal algebraic stacks.

Let us prove part (b). By the lemma 7.1.6 $\Theta_{\mathcal{Z}}$ is representable by a morphism of schemes. Furthermore by lemma 7.1.5 $\Theta_{\mathcal{Z}}$ is a monomorphism of locally noetherian formal algebraic $\operatorname{Spf} k \llbracket \zeta \rrbracket$-stacks, locally formally of finite type. In addition the monomorphism $\Theta_{\mathcal{Z}}$ is surjective by very definition, étale by lemma 7.1.7, and proper by theorem 4.1.12. Hence $\Theta_{\mathcal{Z}}$ is an isomorphism.

Let $\mathcal{Y}$ (resp. $\mathcal{X})$ denote the source (resp. target) of the uniformization morphism.

Lemma 7.1.5. The 1-morphism $\Theta: \mathcal{Y} \rightarrow \mathcal{X}$ is a 1-monomorphism of formal algebraic stacks.

Proof. Consider two $S$-valued points of $\mathcal{Y}$

$$
\underline{x}:=\left(\left(\underline{\mathcal{L}}_{\nu}^{\prime}, \widehat{\tau}_{\nu}^{\prime}\right)_{\nu}, h^{\prime} H\right) \quad \text { and } \quad \underline{y}:=\left(\left(\underline{\mathcal{L}}_{\nu}^{\prime \prime}, \widehat{\tau}_{\nu}^{\prime \prime}\right)_{\nu}, h^{\prime \prime} H\right)
$$

which get mapped to isogenous global $\mathfrak{G}$-shtukas $\left(\underline{\mathcal{G}}^{\prime}, \gamma^{\prime}\right)$ and $\left(\underline{\mathcal{G}}^{\prime \prime}, \gamma^{\prime \prime}\right)$ with level structures, respectively $\gamma^{\prime}$ and $\gamma^{\prime \prime}$, under $\Theta$. Let $\varsigma: \underline{\mathcal{G}}^{\prime} \rightarrow \underline{\mathcal{G}}^{\prime \prime}$ denote this isogeny. By the construction of the morphism $\Theta$, there are canonical isogenies $\bar{\alpha}^{\prime}: \underline{\mathcal{G}}_{\bar{S}}^{\prime} \rightarrow \underline{\mathcal{G}}_{0, \bar{S}}$ and $\bar{\alpha}^{\prime \prime}: \underline{\mathcal{G}}_{\bar{S}}^{\prime \prime} \rightarrow \underline{\mathcal{G}}_{0, \bar{S}}$ see remark 4.1.13. Consider 
the quasi-isogeny $\bar{g}:=\bar{\alpha}^{\prime \prime} \varsigma \bar{\alpha}^{\prime-1}$ in $I(Q)$.

We claim that $\underline{y}=\bar{g} \cdot \underline{x}$. By rigidity of quasi-isogenies, proposition 3.1.3, $\bar{g}: \underline{\mathcal{G}}_{\bar{S}}^{\prime} \rightarrow \underline{\mathcal{G}}_{\bar{S}}^{\prime \prime}$ lifts to $g: \underline{\mathcal{G}}^{\prime} \rightarrow \underline{\mathcal{G}}^{\prime \prime}$ over $S$. Now we let $\varphi_{\nu}$ be the quasi-isogeny defined by the following diagram

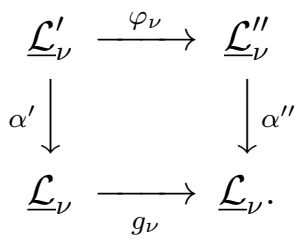

Via the Tannakian duality $g$ operates on the level structure $h^{\prime}$ as an element $\varepsilon(g) \in \mathfrak{G}\left(\mathbb{A}_{Q}\right)=A u t^{\otimes}\left(\omega^{\circ}\right)$, this fits in the diagram

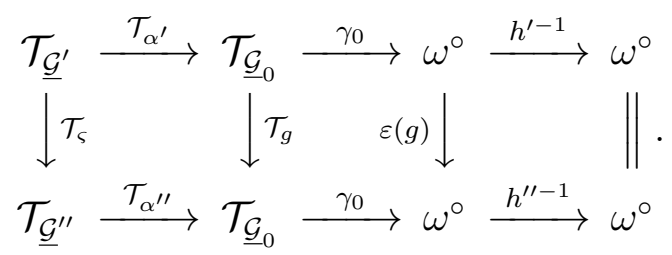

and hence $h^{\prime \prime} H=\varepsilon(g) \cdot h^{\prime} H$. This proves the claim.

Since $\Theta$ is $J(Q)$-invariant this finishes the proof of the first assertion of the lemma.

Lemma 7.1.6. The 1-morphism $\Theta: \mathcal{Y} \rightarrow \mathcal{X}$ is adic. In particular it is representable by a morphism of schemes.

Proof. Let $P: X \rightarrow \mathcal{X}_{\text {red }}$ be a presentation. By theorem 4.1.12 and lemma 7.1 .5 we see that $\mathcal{Y}_{\text {red }} \times \mathcal{X}_{\text {red }} X \rightarrow X$ is a closed immersion. Furthermore since $P$ is an epimorphism we may argue that this is an isomorphism. Hence $\bar{\Theta}: \mathcal{Y}_{\text {red }} \rightarrow \mathcal{X}_{\text {red }}$ is a 1 -isomorphism. Finally since $\mathcal{X}$ and $\mathcal{Y}$ are adic (see proposition 7.1.1) this suffices to show that $\Theta$ is adic. The second assertion follows from the fact that any 1-monomorphism of algebraic stacks is representable by a morphism of schemes, see [L-M] Théorème A.2 and Corollaire 8.1.3.

Lemma 7.1.7. The 1-morphism $\Theta: \mathcal{Y} \rightarrow \mathcal{X}$ is étale.

Proof. Let $Y$ denote the source of the map $\Theta^{\prime}$. Let $\pi: Y \rightarrow \mathcal{Y}$ denote the projection. Let $\mathcal{I}$ be an ideal of definition of $\mathcal{X}$ and let $\overline{\mathcal{X}}:=\mathrm{V}(\mathcal{I})$ be the 
closed substack defined by $\mathcal{I}$. Consider the following 2-cartesian diagram

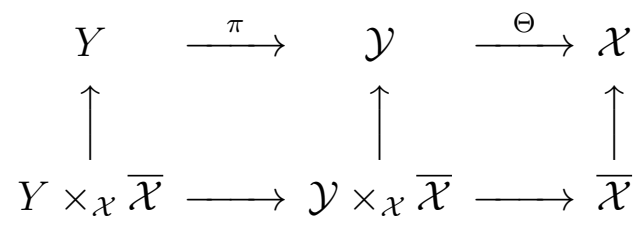

of algebraic Spec $k \llbracket \zeta \rrbracket$-stacks. Now observe that the composition of the morphisms in the top of the above diagram is formally étale. This indeed follows from theorem 4.1.12 and proposition 7.1.1. The 1-morphisms in the bottom row of the diagram are representable by morphisms of finite type between locally noetherian schemes and consequently are étale. Thus we argue that $\mathcal{Y} \times_{\mathcal{X}} \overline{\mathcal{X}} \rightarrow \overline{\mathcal{X}}$ is étale. Now the lemma follows from the fact that both $\Theta$ and the presentation $Y \rightarrow \mathcal{Y}$ are adic see lemma 7.1.6 and proposition 7.1.1. 


\section{Chapter 8}

\section{Discussion about Uniformization and Local Model}

\subsection{Local model for the moduli of global $\mathfrak{G}$ - shtukas}

Recall that a Global affine Grassmannian parametrizes the same tuples of data $\left(\mathcal{G}, \mathcal{G}^{\prime}, c_{1}, \ldots, c_{n}, \varphi\right)$ as Hecke $_{\mathfrak{G}}$ together with a trivialization of $\mathcal{G}^{\prime}$ outside the characteristic sections. One interesting feature of these objects is that they can be viewed as a local model for the moduli of global $\mathfrak{G}$ shtukas (in analogy with the theory of local models for Shimura varieties). Let us state the following theorem

Theorem 8.1.1. For any point y in $\nabla_{n} \mathscr{H}^{1}(C, \mathfrak{G})$ there exist an étale neighborhood of $y$ and a roof

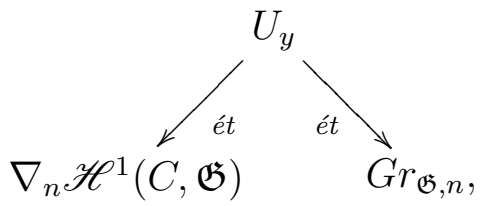

of ind-étale morphisms. In other words the global affine Grassmannian $G r_{\mathfrak{G}, n}$ is a local model for the moduli stack $\nabla_{n} \mathscr{H}^{1}(C, \mathfrak{G})$ of global $\mathfrak{G}$-shtukas. Note that this also induces a roof of étale morphisms after imposing bounds to $\nabla_{n} \mathscr{H}^{1}(C, \mathfrak{G})$ and $G r_{\mathfrak{G}, n}$.

For a constant reductive group $G_{0}$ over $\mathbb{F}_{q}$, this observation was first recorded in $[\mathrm{Var}]$. However there is a mistake in the proof, namely he applies 
the following well-known theorem of Drinfeld and Simpson to construct the étale neighbourhood $U_{y}$ :

Theorem 8.1.2. Let $x$ be a closed point of $C$ and set $\dot{C}:=C \backslash\{x\}$. Let $G_{0}$ be a semi-simple group over a perfect field $k$. Then for any quasi-compact $k$-scheme $S$ and any $G_{0}$-torsor $\mathcal{G}$ on $C \times{ }_{k} S$ the restriction of $\mathcal{G}$ to $\dot{C} \times{ }_{k} S$ is trivial, locally for the fppf-topology on $S$. In addition if $\operatorname{char}(k)$ does not divide the order of the fundamental group $\pi_{1}\left(G_{0}\right)$, then this is even true locally for the étale topology on $S$.

The assumption that $G_{0}$ is semi-simple is necessary (already for the case $G=\mathbb{G}_{m}$ ). In addition this theorem can only be applied under certain circumstances for the characteristic of the ground field.

In this section we modify the proof given in [Var] and produce a proof which is independent of the Drinfeld-Simpson theorem (and can be applied to general $\mathfrak{G}$ ). Finally we briefly mention a link between the uniformization of the moduli stack of global $\mathfrak{G}$-shtukas, we worked out in the previous chapters, and the local model for them.

Proposition 8.1.3. Consider the stacks Hecke $e_{\mathfrak{G}, n}$ and $G r_{\mathfrak{G}, n} \times \mathscr{H}^{1}(C, \mathfrak{G})$ as families over $C^{n} \times \mathscr{H}^{1}(C, \mathfrak{G})$, via the projections $\left(\mathcal{G}, \mathcal{G}^{\prime}, c_{i}, \varphi\right) \mapsto\left(c_{i}, \mathcal{G}^{\prime}\right)$ and $\left(\widetilde{\mathcal{G}}, c_{i}, \widetilde{\varphi}\right) \times \mathcal{G}^{\prime} \mapsto\left(c_{i}, \mathcal{G}^{\prime}\right)$ respectively. They are locally isomorphic with respect to the étale topology on $C^{n} \times \mathscr{H}^{1}(C, \mathfrak{G})$.

Proof. The proof goes in a similar way as [Var, Lem. 4.1], only one has to replace $S$ by $\mathscr{H}^{1}(C, \mathfrak{G})$ and take an étale cover $V \rightarrow C \times_{\mathbb{F}_{q}} \mathscr{H}^{1}(C, \mathfrak{G})$ trivializing the universal $\mathfrak{G}$-bundle over $\mathscr{H}^{1}(C, \mathfrak{G})$ rather than a Zariski trivialization over $S$. Also one sets $U=V \times \mathscr{H}^{1}(C, \mathfrak{G}) \cdots \times \mathscr{H}^{1}(C, \mathfrak{G}) V, U^{\prime}=$ Hecke $_{\mathfrak{G}, n} \times_{C^{n} \times \mathscr{H}^{1}(C, \mathfrak{G})} U, U^{\prime \prime}=G r_{\mathfrak{G}, n} \times_{C^{n}} U, V^{\prime}=V \times_{C \times \mathscr{H}^{1}(C, \mathfrak{G}), \mathcal{G}^{\prime}} C \times U^{\prime}$ and $V^{\prime \prime}=V \times_{C \times \mathscr{H}^{1}(C, \mathfrak{G})} C \times U^{\prime \prime}$.

Proof. of theorem 8.1.1:

Since the curve $C$, the parahoric group $\mathfrak{G}$ and the index $n$ (which stands for the number of characteristic sections) are fixed, we drop them from the notation and simply write $G r=G r_{\mathfrak{G}, n} \mathscr{H}^{1}=\mathscr{H}^{1}(C, \mathfrak{G})$, Hecke $=$ Hecke $_{\mathfrak{G}, n}$ and $\nabla \mathscr{H}^{1}=\nabla_{n} \mathscr{H}^{1}(C, \mathfrak{G})$. Pick an open substack $\mathscr{H}_{\alpha}^{1}(C, \mathfrak{G})$ that contains the image of $y$ under projection to $\mathscr{H}^{1}$, see remark 6.2.19. Let $y^{\prime}$ be the image of $y$ in $C^{n} \times \mathscr{H}_{\alpha}^{1}(C, \mathfrak{G})$ under the projection sending $\left(\mathcal{G}, \mathcal{G}^{\prime}, c_{i}, \varphi\right)$ to $\left(c_{i}, \mathcal{G}^{\prime}\right)$. Take an étale presentation $H_{\alpha}^{1} \rightarrow \mathscr{H}_{\alpha}^{1}(C, \mathfrak{G})$ and let Hecke $_{\alpha}$ denote the base change of Hecke to $H_{\alpha}^{1}$. 


\subsection{LOCAL MODEL FOR THE MODULI OF GLOBAL $\mathfrak{G}-S H T U K A S 79$}

According to proposition 8.1.3, we may pick an étale neighborhood $U \rightarrow C^{n} \times H_{\alpha}^{1} \rightarrow C^{n} \times \mathscr{H}_{\alpha}^{1}$ of $y^{\prime}$, such that the restriction $U^{\prime}$ of Hecke to $U$ and the restriction $U^{\prime \prime}$ of $G r \times \mathscr{H}^{1}$ to $U$ become isomorphic.

Now we claim that the neighborhood $U_{y}:=U^{\prime} \times_{H e c k e} \nabla \mathscr{H}^{1}$ is the desired étale neighborhood.

Consider the following diagram

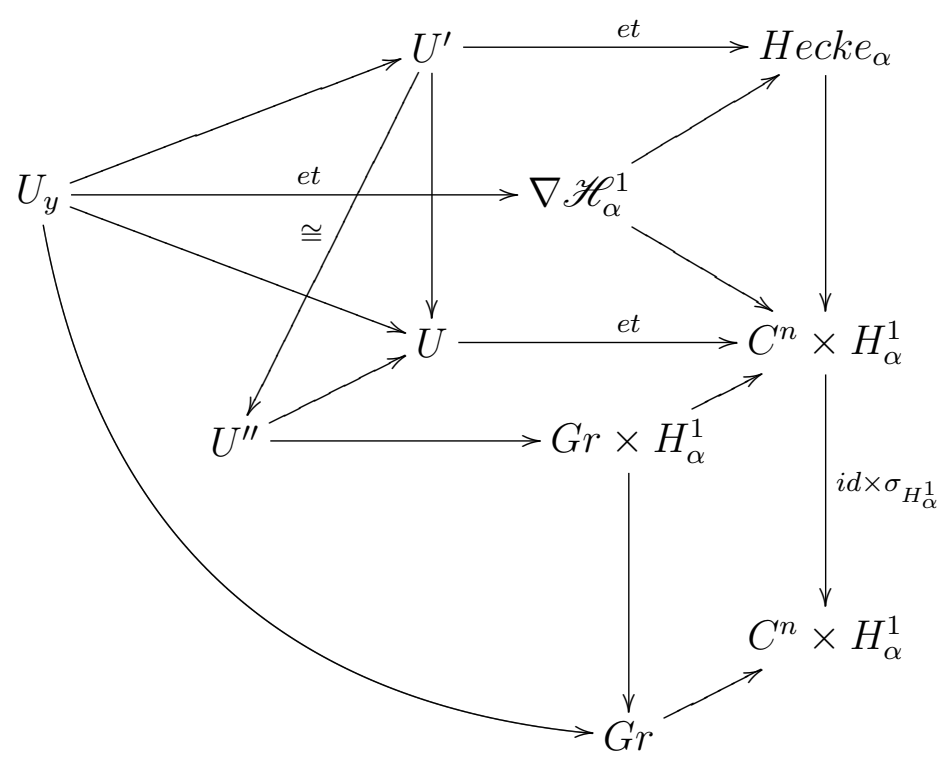

Indeed, the fact that $U_{y}$ is an étale neighbourhood of $G r$ follows from the lemma 8.1.4 applied to the following diagram

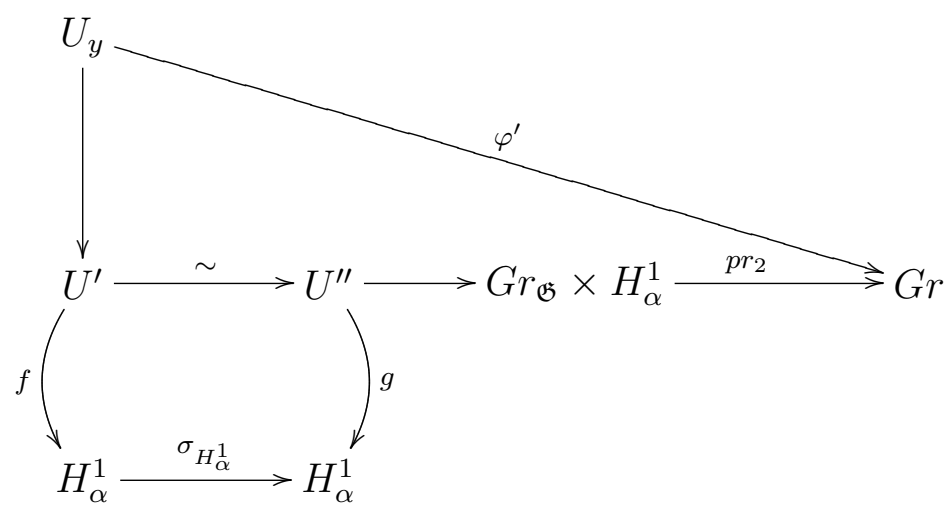

here $f$ is the morphism induced by the projection $\pi:$ Hecke $\rightarrow \mathscr{H}^{1}$ sending $\left(\mathcal{G}, \mathcal{G}^{\prime}, c_{i}, \varphi\right)$ to $\mathcal{G}$ and $g$ is $U^{\prime \prime} \rightarrow G r_{\mathfrak{G}} \times H_{\alpha}^{1}$ followed by the projection. 
80CHAPTER 8. DISCUSSION ABOUT UNIFORMIZATION AND LOCAL MODEL

Lemma 8.1.4. Let $Z$ be a smooth scheme locally of finite type over $\mathbb{F}_{q}$ and let $W, T$ and $Y$ be locally noetherian schemes. Assume that we have a morphism $f: W \rightarrow Z$, an étale morphism $\iota: W \rightarrow Y \times T$ and an isomorphism $\varphi: Y \rightarrow Z$. Let $g: W \rightarrow Z$ denote the morphism $\varphi \circ p r_{1} \circ \iota$, where $\operatorname{pr}_{1}: Y \times T \rightarrow Y$ is the projection to the first factor. Consider the following diagram

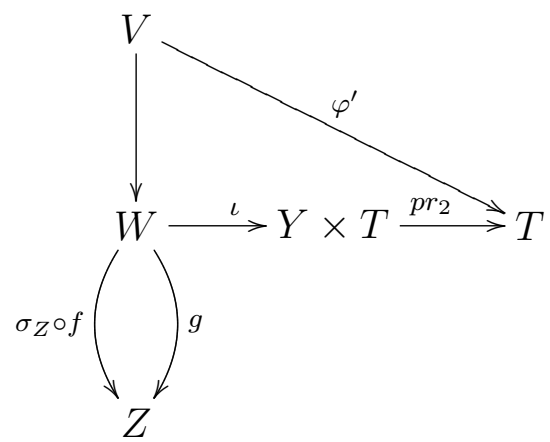

here $V:=\operatorname{ker}\left(\sigma_{Z} \circ f, g: W \rightrightarrows Z\right)$. Then $\varphi^{\prime}$ is étale in either of the following cases

(a) $T$ is smooth over $\mathbb{F}_{q}$,

(b) $Y$ and $T$ are locally of finite type over $\mathbb{F}_{q}$.

Proof. Since the question is local we may reduce to the case that $Z=\mathbb{A}^{m}$.

We first show that the proof of case b) reduces to case a).

We may assume that $T$ is affine. Take a closed embedding $T \hookrightarrow \widetilde{T}$ of $T$ into a smooth affine scheme $\widetilde{T}$ and let $I=I_{T}$ denote the corresponding ideal identifying $T$ as a closed subscheme of $\widetilde{T}$. Let $\widehat{\widetilde{T}}$ denote the spectrum of the ring obtain by taking the completion of the ring $\Gamma\left(\mathcal{O}_{\widetilde{T}}, \widetilde{T}\right)$ with respect to the ideal $I_{T}$. Note that $\widehat{\widetilde{T}}$ is regular, see [EGA, IV, 7.8.3.v. page 215].

Consider the closed immersion $Y \times T \rightarrow Y \times \widehat{\widetilde{T}}$. By [Ra, Chap.V Thm. 1] the étale topology on the closed subscheme $T$ is the induced topology, Zariski locally on $Y \times \widehat{\widetilde{T}}$. Thus we may assume that there is an étale morphism $\widehat{\widetilde{W}} \rightarrow Y \times \widehat{\widetilde{T}}$ such that $W=\widehat{\widehat{W}} \times_{Y \times \widehat{\widetilde{T}}} Y \times T$. Now since $\widehat{\widehat{W}}$ is regular the morphism $f$ extends to $\hat{\tilde{f}}: \widehat{\widehat{W}} \rightarrow Z$. Now assuming the conclusion of the lemma in the case a), we see that the composition

$$
\widetilde{V}:=\operatorname{ker}(\sigma \hat{\tilde{f}}, g: \widehat{\widetilde{W}} \rightrightarrows Z) \rightarrow \widehat{\widetilde{W}} \rightarrow Y \times \widehat{\widetilde{T}} \rightarrow \widehat{\widetilde{T}}
$$


is étale. Therefore its restriction $f: V \rightarrow T$ is also étale.

(a) Assume that $T$ is smooth. This implies that $V$ is étal over $T$. Indeed, $V$ is locally given by $m$ equations with linearly independent differentials inside the smooth scheme $\widetilde{W}$. Then (a) follows by the Jacobi-criterion [BLR, Section 2.2, Prop 7].

\subsection{Generalized Lang Morphism}

Let $y:=\mathcal{G}$ be a global $\mathfrak{G}$-shtuka over $S$. Let $\underline{\nu}:=\nu_{\underline{\mathcal{G}}}$ denote the characteristic of $\mathcal{G}$. Passing to the completion along the characteristic sections, we get the following roof from the local model diagram

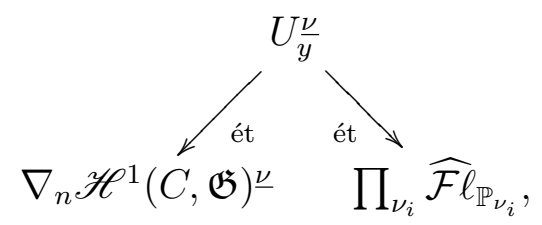

see theorem 8.1.1. We bound this by $\underline{\omega}=\left(\omega_{i}\right)_{i}$. Then, this together with the uniformization morphism $\Theta$, see theorem 7.1.4, induces the following

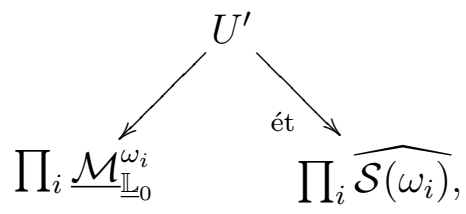

where $U^{\prime}:=U_{\bar{y}}^{\underline{\nu}} \times_{\nabla \mathscr{H} 1, \underline{\underline{\nu}}} \prod_{i} \mathcal{M}_{\mathbb{L}_{0}}^{\omega_{i}}$, and $\widehat{\mathcal{S}\left(\omega_{i}\right)}=\mathcal{S}\left(\omega_{i}\right) \times \operatorname{Spf} k \llbracket \zeta_{i} \rrbracket$. Thus up to a choice of a section for $U^{\prime} \rightarrow \prod_{i} \mathcal{M}_{\mathbb{L}_{0}}^{\omega_{i}}$ we obtain a local morphism from the product of Rapoport-Zink spaces to $\prod_{i} \widehat{\mathcal{S}\left(\omega_{i}\right)}$. Note that $\prod_{i} \widehat{\mathcal{S}\left(\omega_{i}\right)}$ can be viewed as a parameter space for Hodge-Pink structures (see [Ha2]). Consequently one may naturally pose the question "how the period morphism contributes to this picture?" We leave the interpretation and precise formulation of this observation unanswered. 
82CHAPTER 8. DISCUSSION ABOUT UNIFORMIZATION AND LOCAL MODEL 


\section{Bibliography}

[BD] A. Beilinson, V. Drinfeld: Quantization of Hitchin's integrable system and Hecke eigensheaves, preprint on http://www. math.uchicago.edu/ mitya/langlands.html.

[BH] M. Bornhofen, U. Hartl: Pure Anderson Motives and Abelian $\tau$-Sheaves over Finite Fields Preprint January 2010, arXiv:math.NT/0609733 .

[BL] A. Beauville and Y. Laszlo: Un lemme de descente, Comptes Rendus Acad. Sci. Paris, vol. 320, série I (1995), 335-340.

[BL1] A. Beauville, Y. Laszlo: Conformal blocks and generalized theta functions. Commun. Math. Phys. 164 (1994), 385419.

[Beh] K. Behrend. The lefschetz trace formula for the moduli stack of principal bundles. PhD thesis, UC Berkeley.

[BLS] A. Beauville, Y. Laszlo, C. Sorger: The Picard group of the moduli of G-bundles on a curve. Compositio Math. 112 (1998), 183216.

[BT1] F. Bruhat, J. Tits: Groupes réductifs sur un corps local, Inst. Hautes Études Sci. Publ. Math. 41 (1972), 5251.

[BT2] F. Bruhat, J. Tits: Groupes réductifs sur un corps local. II. Schémas en groupes. Existence dúne donné radicielle valuée. Inst. Hautes Études Sci. Publ. Math. 60 (1984), 197376.

[BLR] S. Bosch, W. Lutkebohmert, M. Raynaud: Néron models, Ergebnisse der Mathematik und ihrer Grenzgebiete (3), 21. Springer-Verlag, Berlin (1990).

[Dr1] V. G. Drinfeld, Moduli varieties of F-sheaves, Func. Anal. and Appl. 21 (1987), 107-122.

[Dr2] V. G. Drinfeld: Coverings of p-adic symmetric domains, Funct. Anal. Appl. 10 (1976), 107-115. 
[EGA] A. Grothendieck: Élements de Géométrie Algébrique, Publ. Math. IHES , Bures-Sur-Yvette, 1960-1967; see also Grundlehren 166, Springer-Verlag, Berlin etc. 1971.

[SGA] A. Grothendieck: Revêtements étales et groupe fondamental, LNM 224, Springer-Verlag, Berlin-Heidelberg 1971.

[Ga] D. Gaitsgory, Construction of central elements in the affine Hecke algebra via nearby cycles, Invent. Math. 144 (2001), 253280.

[H-R] T. Haines and M. Rapoport: On parahoric subgroups appendix to [PR1].

[Ha1] U. Hartl: Uniformizing the Stacks of Abelian Sheaves, in Number Fields and Function fields - Two Parallel Worlds, Papers from the 4th Conference held on Texel Island, April 2004, Progress in Math. 239, Birkhauser-Verlag, Basel 2005, pp. 167222.

[Ha2] U. Hartl: Period Spaces for Hodge Structures in Equal Characteristic, to appear in Ann. of Math. (2010); see also arXiv:math.NT/0511686.

$[\mathrm{H}-\mathrm{V}] \quad \mathrm{U}$. Hartl E. Viehmann, The Newton stratification on deformations of local G-shtukas Journal fr die reine und angewandte Mathematik (Crelle) (2010),

[He] J. Heinloth: Uniformization of $\mathcal{G}$-bundles, Math. Ann. 347 (2010), 499528.

[L] L. Lafforgue, Chtoucas de Drinfeld et correspondance de Langlands, Invent. Math. 147 (2002), 1-241.

[La1] E. Landvogt: Some Functorial Properties of Bruhar-Tits Buildings, J. reine angew. math. 518 (2000), 213?241.

[La2] E. Landvogt: A Compactification of the Bruhat-Tits Building, Lecture Notes in Mathematics 1619 (1996), Springer-Verlag.

[Lan] S. Lang, Algebraic groups over finite fields, Amer. J. Math. 78 (1956), 555-563.

[L-M] G. Laumon, L. Moret-Bailly: Champs algebriques, Ergeb. Math. Grenzgebiete 39, Springer, Berlin, 2000.

[Ja] J. C. Jantzen: Representations of algebraic groups, Mathematical Surveys and Monographs 107, AMS, Providence, RI, 2003. 
[Kat] N. M. Katz, p-adic properties of modular schemes and modular forms, in Modular Functions of One Variable, III (Proc. Internat. Summer School, Univ. Antwerp, Antwerp, 1972), Lecture Notes in Math 350, Springer- Verlag, New York, 1973, pp. 69-190. MR 0447119. Zbl 0271.10033 .

[Ko1] R. E. Kottwitz: Isocrystals with additional structure, Compositio Math. 56 (1985), no. 2, 201-220.

[Ko2] R. E. Kottwitz: Isocrystals with additional structure II, Compositio Math. 109 (1997), no. 3, 255-339.

[PR1] G. Pappas, M. Rapoport: Local models in the ramified case I, The EL-case, J. Algebraic Geom. 12 (2003), no. 1, 107-145.

[PR2] G. Pappas, M. Rapoport: Twisted loop groups and their affine flag varieties, Advances in Mathematics 219 (2008), 118-198.

[PR3] G. Pappas, M. Rapoport Some questions about $\mathcal{G}$-bundles on curves. Algebraic and Arithmetic Structure of Moduli Spaces, Advanced Studies in Pure Mathematics 58, (2010), 159-171.

[RZ] M. Rapoport, T. Zink: Period Spaces for p-divisible Groups, Ann. Math. Stud. 141, Princeton University Press, Princeton 1996.

[RZ2] M. Rapoport, T. Zink: A finiteness theorem in the Bruhat-Tits building: an application of Landvogt's embedding theorem, Indag. Mathem., N.S. 10, 449-458.

[Ra] M. Raynaud, Anneaux locaux henséliens, Lect. Notes Math. 169, Springer, 1970.

[Ri] T. Richarz: Schubert varieties in twisted affine flag varieties and local models. arXiv:1011.5416v1

[Var] Y. Varshavsky, Moduli Spaces of principal F-bundles, Selecta Math. (N.S.) 10 (2004), no. 1, 131166.

[Wed] T. Wedhorn, On Tannakian duality over valuation rings, J. Algebra 282 (2004), no. 2, 575609. MR MR2101076 (2005j:18007) 